\title{
FETI-DP DOMAIN DECOMPOSITION METHODS FOR ELASTICITY WITH STRUCTURAL CHANGES: P-ELASTICITY
}

\author{
Axel Klawonn ${ }^{1}$, Patrizio NefF ${ }^{1}$, Oliver Rheinbach ${ }^{1}$ and Stefanie Vanis ${ }^{1}$
}

\begin{abstract}
We consider linear elliptic systems which arise in coupled elastic continuum mechanical models. In these systems, the strain tensor $\varepsilon_{P}:=\operatorname{sym}\left(P^{-1} \nabla u\right)$ is redefined to include a matrix valued inhomogeneity $P(x)$ which cannot be described by a space dependent fourth order elasticity tensor. Such systems arise naturally in geometrically exact plasticity or in problems with eigenstresses. The tensor field $P$ induces a structural change of the elasticity equations. For such a model the FETI-DP method is formulated and a convergence estimate is provided for the special case that $P^{-T}=\nabla \psi$ is a gradient. It is shown that the condition number depends only quadratic-logarithmically on the number of unknowns of each subdomain. The dependence of the constants of the bound on $P$ is highlighted. Numerical examples confirm our theoretical findings. Promising results are also obtained for settings which are not covered by our theoretical estimates.
\end{abstract}

Mathematics Subject Classification. 65F10, 65N30, 65N55.

Received November 21, 2009. Revised July 22, 2010.

Published online November 30, 2010.

\section{INTRODUCTION}

This paper deals with the efficient solution of problems of the form

$$
\int_{\Omega} 2 \mu_{e}\left\langle\operatorname{sym}\left(P^{-1} \nabla u\right), \operatorname{sym}\left(P^{-1} \nabla v\right)\right\rangle+\lambda_{e} \operatorname{tr}\left[P^{-1} \nabla u\right] \operatorname{tr}\left[P^{-1} \nabla v\right] \mathrm{d} \mathbf{x}=(F, v)_{L_{2}(\Omega)},
$$

by an appropriate FETI-DP domain decomposition method $[16,18,19,21-23,26,29,30,33,36,37,55]$. The system (1.1) reduces to the standard linear elastic case if the $3 \times 3$-matrix $P=\mathrm{Id}$. However, generally, it cannot be reduced to standard linear elasticity. Throughout this paper we will denote the problem (1.1) by $P$-elasticity.

Domain decomposition methods are an efficient approach for the parallel solution of elliptic partial differential equations. By domain decomposition methods, we understand preconditioned iterative algorithms for the large linear systems arising from the discretization of partial differential equations. In such methods, the domain, on which the partial differential equation has to be solved, is decomposed into a number of smaller subdomains. Here, we will only consider nonoverlapping subdomains. In each step of the iterative method and for each

Keywords and phrases. FETI-DP, plasticity, eigenstresses, inhomogeneity, extended elasticity, structural changes, micromorphic model.

1 Fakultät für Mathematik, Universität Duisburg-Essen, Campus Essen, Universitätsstraße 3, 45117 Essen, Germany. axel.klawonn@uni-duisburg-essen.de; patrizio.neff@uni-duisburg-essen.de; oliver.rheinbach@uni-duisburg-essen.de; stefanie.vanis@uni-duisburg-essen.de 
subdomain, a local problem is solved; often it is a restriction of the original problem to the subdomains, neglecting for the moment that the boundary conditions are usually different for the local problems and the original boundary value problem. In addition to the local solutions, a small global problem has to be solved to guarantee the parallel scalability of the domain decomposition method which is necessary in order to exploit efficiently a growing number of processors of a parallel computer. For an extensive introduction to different domain decomposition methods, we refer to the monographies by Smith et al. [53], Toselli and Widlund [55], and Quarteroni and Valli [49]. In the present article, we consider a special class of nonoverlapping domain decomposition methods which belong to the family of dual-primal Finite Element Tearing and Interconnecting (FETI-DP) algorithms. These algorithms are parallel iterative substructuring methods that descended from the earlier one-level and two-level FETI algorithms; see [12-15,17]. For a recent overview of different FETI methods, see also Klawonn and Rheinbach [24].

In this paper, for the first time, we develop and analyze FETI-DP domain decomposition methods for the $P$-elasticity problem, extending the results obtained in [26] to $P$-elasticity. In an extensive numerical study we confirm the new condition number estimate.

The motivation for the $P$-elasticity problem originates from a finite visco-elasto-plasticity model based on the multiplicative decomposition of the deformation gradient; see [39]. The $P$-elasticity problem appears naturally as one of two subproblems when solving a related finite elasticity problem by a staggered scheme, cf. [32]. A motivation for the $P$-elasticity problem in structural mechanics is given in Section 2; this section may be left out at a first reading.

In this article we will use Id for the identity matrix and the following further notation, where $X, Y \in$ $\mathbb{R}^{n \times m}, n, m \in \mathbb{N}$,

$$
\begin{aligned}
& \operatorname{sym}(X):=\frac{1}{2}\left(X+X^{T}\right) ; \operatorname{skew}(X):=\frac{1}{2}\left(X-X^{T}\right) ; \operatorname{tr}(X):=\sum_{i=1}^{n} X_{i i} ;(X, Y)_{L_{2}(\Omega)}:=\int_{\Omega}\langle X, Y\rangle \mathrm{d} \mathbf{x} \\
& \operatorname{Div}(X):=\left(\sum_{j=1}^{m} \frac{\partial X_{1 j}}{\partial x_{j}}, \ldots, \sum_{j=1}^{m} \frac{\partial X_{n j}}{\partial x_{j}}\right)^{T} ;\langle X, Y\rangle:=\operatorname{tr}\left(X^{T} Y\right)=\sum_{i=1}^{n} \sum_{j=1}^{m} X_{i j} Y_{i j} ;\|X\|^{2}:=\langle X, X\rangle .
\end{aligned}
$$

The remainder of this article is organized as follows. In Section 2, a derivation of the equations of $P$-elasticity from a nonlinear system of partial differential equations is given. In Section 3, the equations of linear $P$-elasticity in three dimensions are presented, a basis for their null space is computed, and the discretization by piecewise quadratic finite elements is discussed. In Section 4, the basic FETI-DP algorithm is described, and in Section 5, the selection of constraints for our FETI-DP algorithms is discussed. In Section 6, different Korn-type inequalities, which are needed in our convergence analysis, are presented. The condition number estimate, which is central to the convergence analysis of our FETI-DP methods, is given in Section 7. In Section 8, computational results are presented which numerically confirm our theoretical findings; promising numerical results are also presented for cases which are not covered by our theory. In the Appendix A, some auxiliary technical lemmas, which are needed in our convergence analysis in Section 7, are collected and proofs are given for some of them for the case of piecewise quadratic finite elements.

\section{Motivation of the P-Elasticity problem}

The finite element theory is well developed for classical linear elasticity, also with variable coefficients. The convergence estimates for iterative solvers, e.g., FETI-DP methods, usually rely on the underlying finite element theory. Unfortunately, from the point of view of structural mechanics, the equations of linear elasticity only have a limited range of applications due to the simplifications inherent in the theory which make it impossible to treat cases where large rotations appear. In order to overcome the limitations of linear elasticity it is necessary to consider nonlinear (or finite) elasticity. Here, large rotations are consistently covered; such models are then called geometrically exact. This feature, however, immediately destroys convexity. It may also be the case that large deformations and high stresses occur, in which a so called nonlinear physical relation needs to be mapped. 
In Ball $[4,5]$ the crucial polyconvexity condition was introduced, and it was realized that it is consistent with frame-indifference, i.e., the geometrical exactness. Using this condition, existence of absolute minimizers can be established by the direct methods of the calculus of variations under rather mild assumptions. Recently, this concept has also been generalized to anisotropic material response $[7,50-52]$ thus giving a partial answer to Problem 2 posed in [6] by Ball "Are there ways of verifying polyconvexity and quasiconvexity for a useful class of anisotropic stored-energy functions?".

A drawback of the method, however, is that it does not give any information about local equilibria. Indeed, a "nice" polyconvex model may have a multitude of local and global equilibria as the energy landscape can be quite complicated [54].

In finite element calculations for nonlinear elasticity, usually a homotopy-method is used, i.e., the loads are increased in small load steps, together with a Newton linearization. The sequence of linear problems to be solved for the increments of the solution will, in general, not be uniformly positive definite and therefore little or nothing can be said about smoothness of solutions and convergence rates of the nonlinear and linear iteration. Typically, one needs thus to assume that the stationary solution lies in a uniform potential well and that this solution is smooth. In that case, the linear subproblems are well-posed and one is basically in the same situation as in linear elasticity (with a modified configuration in the vicinity of which the linearization is a bijection [56]) and one can apply the standard finite element framework developed for the linear theory. Theorems in this spirit may be found in Le Tallec [34]. Unfortunately, the assumptions made in these statements cannot be verified a priori in general, limiting the practical value of these studies. The situation is basically the same for nonlinear elasticity, nonlinear viscoelasticity, and nonlinear elasto-plasticity where even other fields than that of the displacements need to be computed.

In many applications the necessary amount of nonlinearity is embodied by the geometrical exactness of the model, e.g., in elasto-plasticity, and in viscoelasticity of metals (small strain, large deformations). Here, the physical nonlinearity can be ignored. The physical nonlinearity cannot be ignored, however, in arterial walls [9] or rubber elasticity, cases which we exclude in the present investigations.

The question arises how to use the extra bit of information of small elastic strain in conjunction with finite element methods. By introducing additional field variables, which either obey their own evolution equations $[39,40,57]$ or have their own balance equations [44,45] it is possible to set up a class of models which are on the one hand geometrically exact, on the other hand lead to elastic balance equations for the traditional displacement increments which are second order linear elliptic systems. A further advantage of these models is that in case of the ODE-augmented model, the coupled system is known to have a unique solution [41,42], in case of additional balance equations the system admits a global minimizer [43].

To be more specific, the micromorphic model [43] allows to model structural changes of the material. This problem consists basically of the frame-indifferent two-field minimization problem for the classical deformation $\varphi: \Omega \subset \mathbb{R}^{3} \mapsto \mathbb{R}^{3}$ and the additional matrix valued field $P: \Omega \subset \mathbb{R}^{3} \mapsto \mathrm{GL}^{+}(3)$, here $X \in \mathrm{GL}^{+}(3)$ are the invertible $3 \times 3$-matrices with positive determinants,

$$
\int_{\Omega} \widehat{W}(\nabla \varphi, P)-\langle f, \varphi\rangle \mathrm{d} \mathbf{x} \mapsto \min _{(\varphi, P)}
$$

where the energy density is given by

$$
\widehat{W}(\nabla \varphi, P)=\mu_{e}\left\|\operatorname{sym}\left(P^{-1} \nabla \varphi-\mathrm{Id}\right)\right\|^{2}+\frac{\lambda_{e}}{2} \operatorname{tr}\left[\operatorname{sym}\left(P^{-1} \nabla \varphi-\mathrm{Id}\right)\right]^{2} .
$$

Here it is understood that $P: T_{x} \Omega \mapsto T_{\varphi(x)} \varphi(\Omega)$ is a two point field such that $P^{-1} \nabla \varphi: T_{x} \Omega \mapsto T_{x} \Omega$ is a second order tensor for which symmetrization $\operatorname{sym}(X)$ makes sense; moreover, the density is invariant w.r.t. the transformation

$$
(\varphi, P) \mapsto(\bar{Q} \varphi, \bar{Q} P)
$$

for any rigid rotation $\bar{Q} \in \mathrm{SO}(3)$. This is the desired frame-indifference. 
In a next step, we decouple the computation of the two fields $\varphi$ and $P$ by proposing an appropriate staggered scheme. We first compute $\varphi$ with a given $P$ and then update $P$ through another method. The first step, where $P$ is given as a field variable, leads to a linear elliptic system of equations with variable coefficients, determined by $P$. It has the form

$$
\int_{\Omega} 2 \mu_{e}\left\langle\operatorname{sym}\left(P^{-1} \nabla \varphi-\mathrm{Id}\right), \operatorname{sym}\left(P^{-1} \nabla v\right)\right\rangle+\lambda_{e} \operatorname{tr}\left[P^{-1} \nabla \varphi-\mathrm{Id}\right] \operatorname{tr}\left[P^{-1} \nabla v\right]-\langle f, v\rangle \mathrm{d} \mathbf{x}=0
$$

for all test functions $v \in H_{0}^{1}\left(\Omega, \partial \Omega_{D}\right):=\left\{v \in H^{1}(\Omega): v=0\right.$ on $\left.\partial \Omega_{D}\right\}$. Using the notation $\nabla \varphi=\nabla \varphi_{\mathrm{old}}+\nabla u$, we obtain

$$
\begin{aligned}
& \int_{\Omega} 2 \mu_{e}\left\langle\operatorname{sym}\left(P^{-1} \nabla u\right), \operatorname{sym}\left(P^{-1} \nabla v\right)\right\rangle+\lambda_{e} \operatorname{tr}\left[P^{-1} \nabla u\right] \operatorname{tr}\left[P^{-1} \nabla v\right]-\langle f, v\rangle \\
& \quad+2 \mu_{e}\left\langle\operatorname{sym}\left(P^{-1} \nabla \varphi_{\text {old }}-\mathrm{Id}\right), \operatorname{sym}\left(P^{-1} \nabla v\right)\right\rangle+\lambda_{e} \operatorname{tr}\left[P^{-1} \nabla \varphi_{\text {old }}-\operatorname{Id}\right] \operatorname{tr}\left[P^{-1} \nabla v\right] \mathrm{d} \mathbf{x}=0 .
\end{aligned}
$$

Assuming that $\varphi_{\text {old }}$ is already given, we collect the known terms by defining for any term on $H^{1}(\Omega)$

$$
-(F, v)_{L_{2}(\Omega)}:=\int_{\Omega} 2 \mu_{e}\left\langle\operatorname{sym}\left(P^{-1} \nabla \varphi_{\text {old }}-\mathrm{Id}\right), \operatorname{sym}\left(P^{-1} \nabla v\right)\right\rangle+\lambda_{e} \operatorname{tr}\left[P^{-1} \nabla \varphi_{\text {old }}-\operatorname{Id}\right] \operatorname{tr}\left[P^{-1} \nabla v\right]-\langle f, v\rangle \mathrm{d} \mathbf{x} .
$$

Then the problem reads: find the increment $u$ such that

$$
\int_{\Omega} 2 \mu_{e}\left\langle\operatorname{sym}\left(P^{-1} \nabla u\right), \operatorname{sym}\left(P^{-1} \nabla v\right)\right\rangle+\lambda_{e} \operatorname{tr}\left[P^{-1} \nabla u\right] \operatorname{tr}\left[P^{-1} \nabla v\right] \mathrm{d} \mathbf{x}=(F, v)_{L_{2}(\Omega)}
$$

for all $v \in H_{0}^{1}\left(\Omega, \partial \Omega_{D}\right)$.

This system can, in general, not be reduced to linear elasticity with variable coefficients, where we would rather have

$$
\int_{\Omega}\langle\mathbb{C}(x) \cdot \operatorname{sym} \nabla u, \operatorname{sym} \nabla v\rangle \mathrm{d} \mathbf{x}=(f, v)_{L_{2}(\Omega)} .
$$

Here, the variable coefficients only change the fourth order elasticity tensor $\mathbb{C}(x): \operatorname{Sym}(3) \rightarrow \operatorname{Sym}(3)$ without necessarily destroying positive definiteness in the classical linearized $\operatorname{strain} \varepsilon=\operatorname{sym}(\nabla \varphi-\mathrm{Id})=\operatorname{sym} \nabla u$. In our case, however, we need to replace the linear strain tensor by a modified strain tensor, $P$-strain, say, having the form

$$
\varepsilon_{P}:=\operatorname{sym}\left(P^{-1} \nabla u\right)
$$

It is easily observed that for $P=\mathrm{Id}$ and $\varphi_{\text {old }}(x)=x$ we recover the bilinear formulation of linear elasticity and this is consistent with using $\varepsilon_{P}$ as a strain measure.

Remark 2.1 (choice of $\varepsilon_{P}$ ). If we had chosen instead $\operatorname{sym}\left(\nabla u P^{-1}\right)$ as the relevant strain measure, then the ensuing analysis in case that $P=\nabla \psi$ is a gradient would be nothing else than the classical linear elasticity problem posed on the transformed domain $\psi(\Omega)$. However, $\operatorname{sym}\left(\nabla u P^{-1}\right)$ is not frame-indifferent $(2.3)$.

In this paper we will restrict ourselves to the subproblem of $P$-elasticity and will consider the coupled nonlinear problem elsewhere. Note that the solution of the first $P$-elastic step in the staggered scheme is unique and smooth if Dirichlet-boundary conditions are prescribed everywhere for $\varphi$ (hence $u$ ) and $P$ is given, invertible and smooth [42]. This is based on a generalized Korn inequality, adapted to this case [38,47].

A conceptually simpler situation arises if $P$ is a gradient, i.e., if $P=\nabla \psi$ for some diffeomorphism $\psi: \Omega \subset$ $\mathbb{R}^{3} \mapsto \mathbb{R}^{3}$. This is not the case for the plasticity problem mentioned above or in Cosserat models $(P=\bar{R} \in$ $\mathrm{SO}(3))$ and micromorphic models $\left(P \in \mathrm{GL}^{+}(3)\right)[44-46]$, but it is applicable when considering a 3D-curved 


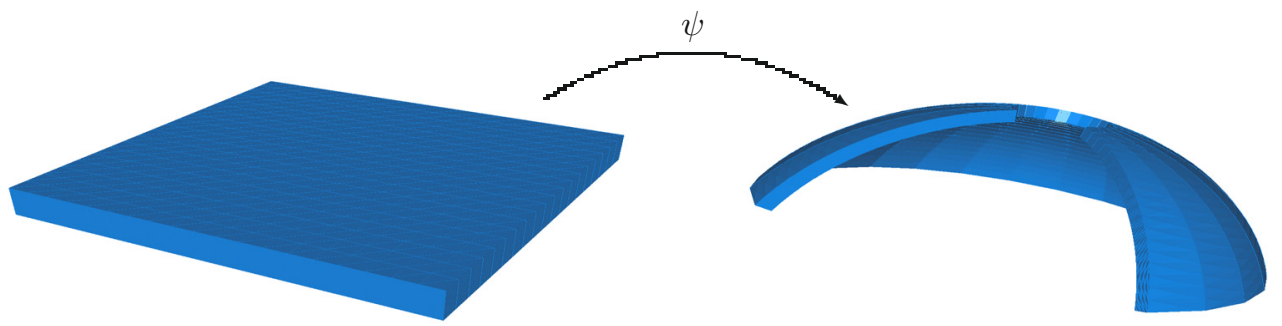

$$
\psi(\mathbf{x})=\left(\begin{array}{c}
((1-h)+x h) \cos (1.5 \pi y) \cos (\alpha+10 z)(\beta-\alpha) \\
((1-h)+x h) \sin (1.5 \pi y) \cos (\alpha+10 z)(\beta-\alpha) \\
((1-h)+x h) \sin (\alpha+10 z)(\beta-\alpha)
\end{array}\right)
$$

Figure 1. $P$-elasticity: Predeformation induced by a function $\Psi$ and a resulting $P=\nabla \Psi$. The parameters $\alpha$ and $\beta$ represent angles of the dome and $h$ its thickness.

shell problem. Then $\psi$ can be identified with the stress-free predeformation of a flat reference surface into the truly curved initial surface of the shell, see Figure 1 , and $\varepsilon_{P}=\operatorname{sym}\left(P^{-1} \nabla u\right)$ is a measure of the elastic energy of the shell.

In the analysis of our algorithm, we restrict ourselves for technical reasons to another gradient case, namely $P^{-T}=\nabla \psi$ because it allows us to understand and analyze the effect which $P$ introduces, in a natural way. We strongly believe that our results are also generically true for more general non gradient fields for which $P^{-T} \neq \nabla \psi$.

\section{The equations of P-elasticity}

The equations of linear elasticity model the displacement of a linear elastic material under the action of external and internal forces. The standard equations of linear elasticity consider only the displacement of the body and disregard structural changes of the material. In order to achieve a more accurate model, we have introduced the parameter $P \in \mathbb{R}^{3 \times 3}$, see above. Here, $P=P(x), x \in \Omega$, is a tensorial field which, in general, does not have the form $P^{-T}=\nabla \psi$. If $P$ is the identity, (3.1) reduces to the standard formulation of linear elasticity.

The elastic body occupies a domain $\Omega \subset \mathbb{R}^{3}$ which is assumed to be Lipschitz, connected, and of diameter 1 . Its boundary is denoted by $\partial \Omega$ and it is assumed that a part of it, denoted as $\partial \Omega_{D}$, is clamped, i.e., with homogeneous Dirichlet boundary conditions, and that the rest, denoted as $\partial \Omega_{N}:=\partial \Omega \backslash \partial \Omega_{D}$, is subject to a surface force $\mathbf{g}$, i.e., a natural boundary condition. There is also the possibility of introducing a body force $\mathbf{f}$, e.g., gravity. With $\mathbf{H}^{\mathbf{1}}(\Omega):=\left(H^{1}(\Omega)\right)^{3}$, the appropriate space for our variational formulation is the Sobolev space $\mathbf{H}_{\mathbf{0}}^{\mathbf{1}}\left(\Omega, \partial \Omega_{D}\right)$. If the new structural parameter $P$ is supposed to be given, the problem reduces to compute the displacement $\mathbf{u} \in \mathbf{H}_{\mathbf{0}}^{\mathbf{1}}\left(\Omega, \partial \Omega_{D}\right)$ of the elastic body $\Omega$ such that for all $\mathbf{v} \in \mathbf{H}_{\mathbf{0}}^{\mathbf{1}}\left(\Omega, \partial \Omega_{D}\right)$, we have

$$
\int_{\Omega} 2 \mu_{e}(\mathbf{x}) \varepsilon_{P}(\mathbf{u}): \varepsilon_{P}(\mathbf{v}) \mathrm{d} \mathbf{x}+\int_{\Omega} \lambda_{e}(\mathbf{x}) \operatorname{tr}\left(P^{-1} \nabla \mathbf{u}\right) \operatorname{tr}\left(P^{-1} \nabla \mathbf{v}\right) \mathrm{d} \mathbf{x}=(\mathbf{F}, \mathbf{v})_{L_{2}(\Omega)}
$$

where $\mu_{e}$ and $\lambda_{e}$ are the Lamé constants which are related to the Young modulus $E$ and the Poisson ratio $\nu$ through

$$
\mu_{e}=\frac{E}{2(1+\nu)}, \quad \lambda_{e}=\frac{E \nu}{(1+\nu)(1-2 \nu)}
$$


and we assume $\mu_{e}>0, \lambda_{e} \geq 0$ throughout. With

$$
\varepsilon_{P}(\mathbf{u}):=\operatorname{sym}\left(P^{-1} \nabla \mathbf{u}\right) \quad \text { and } \quad\left(\varepsilon_{P}(\mathbf{u})\right)_{i j}=\frac{1}{2}\left(\sum_{k=1}^{3}\left(P^{-1}\right)_{i k} \frac{\partial u_{k}}{\partial x_{j}}+\frac{\partial u_{k}}{\partial x_{i}}\left(P^{-1}\right)_{j k}\right)
$$

we have

$$
\begin{aligned}
\varepsilon_{P}(\mathbf{u}): \varepsilon_{P}(\mathbf{v})= & \sum_{i, j=1}^{3}\left(\varepsilon_{P}\right)_{i j}(\mathbf{u}) \cdot\left(\varepsilon_{P}\right)_{i j}(\mathbf{v}) \\
(\mathbf{F}, \mathbf{v})_{L_{2}(\Omega)}:= & \int_{\Omega}\langle\mathbf{f}, \mathbf{v}\rangle \mathrm{d} \mathbf{x}+\int_{\partial \Omega_{N}}\langle\mathbf{g}, \mathbf{v}\rangle \mathrm{d} \sigma-\int_{\Omega} \mu_{e}(\mathbf{x})\left\langle P^{-T}\left(P^{-1} \nabla \varphi_{\text {old }}+\left(\nabla \varphi_{\text {old }}\right)^{T} P^{-T}-2 \mathrm{Id}\right), \nabla \mathbf{v}\right\rangle \mathrm{d} \mathbf{x} \\
& -\int_{\Omega} \lambda_{e}(\mathbf{x}) \operatorname{tr}\left(P^{-1} \nabla \varphi_{\text {old }}-\mathrm{Id}\right) \operatorname{tr}\left(P^{-1} \nabla \mathbf{v}\right) \mathrm{d} \mathbf{x} .
\end{aligned}
$$

By using this notation the bilinear form associated with linear $P$-elasticity is given by

$$
a(\mathbf{u}, \mathbf{v})=2\left(\mu_{e}(\mathbf{x}) \varepsilon_{P}(\mathbf{u}), \varepsilon_{P}(\mathbf{v})\right)_{L_{2}(\Omega)}+\left(\lambda_{e}(\mathbf{x}) \operatorname{tr}\left(P^{-1} \nabla \mathbf{u}\right), \operatorname{tr}\left(P^{-1} \nabla \mathbf{v}\right)\right)_{L_{2}(\Omega)} .
$$

We will also use the standard Sobolev space norm

$$
\|\mathbf{u}\|_{H^{1}(\Omega)}:=\left(|\mathbf{u}|_{H^{1}(\Omega)}^{2}+\|\mathbf{u}\|_{L_{2}(\Omega)}^{2}\right)^{1 / 2}
$$

with $\|\mathbf{u}\|_{L_{2}(\Omega)}^{2}:=\sum_{i=1}^{3} \int_{\Omega}\left|u_{i}\right|^{2} \mathrm{~d} \mathbf{x}$ and $|\mathbf{u}|_{H^{1}(\Omega)}^{2}:=\sum_{i=1}^{3}\left\|\nabla u_{i}\right\|_{L_{2}(\Omega)}^{2}$. Since the two terms of the $H^{1}$-norm scale differently under dilation of $\Omega$ we introduce the factor $\frac{1}{H^{2}}$ in front of the squared $L_{2}$-norm where $H$ is the diameter of $\Omega$. With these definitions we can show that the bilinear form $a(\cdot, \cdot)$ is continuous with respect to $\|\cdot\|_{H^{1}(\Omega)}$. We can estimate the two terms occurring in (3.2) by assuming that $P, P^{-1} \in \mathcal{C}^{0}(\bar{\Omega})$ and using for $A, B \in \mathbb{R}^{n \times n}$

- the Cauchy-Schwarz inequality: $(A, B)_{L_{2}(\Omega)} \leq\|A\|_{L_{2}(\Omega)}\|B\|_{L_{2}(\Omega)}$;

- the submultiplicativity of the $L_{2}$-norm: $\|A B\|_{L_{2}(\Omega)} \leq\|A\|_{L_{2}(\Omega)}\|B\|_{L_{2}(\Omega)}$;

- $\left\|A^{T}\right\|_{L_{2}(\Omega)}=\|A\|_{L_{2}(\Omega)}$;

- $\left(A^{T}, B\right)_{L_{2}(\Omega)}=\left(A, B^{T}\right)_{L_{2}(\Omega)}$;

- $\|\nabla \mathbf{u}\|_{L_{2}(\Omega)}=|\mathbf{u}|_{H^{1}(\Omega)} \leq\|\mathbf{u}\|_{H^{1}(\Omega)}$.

For the first term in (3.2) this leads to

$$
\begin{aligned}
\left(\varepsilon_{P}(\mathbf{u}), \varepsilon_{P}(\mathbf{v})\right)_{L_{2}(\Omega)} & \leq \frac{1}{4}\left(2\left\|P^{-1} \nabla \mathbf{u}\right\|_{L_{2}(\Omega)}\left\|P^{-1} \nabla \mathbf{v}\right\|_{L_{2}(\Omega)}+2\left\|P^{-1} \nabla \mathbf{u}\right\|_{L_{2}(\Omega)}\left\|(\nabla \mathbf{v})^{T} P^{-T}\right\|_{L_{2}(\Omega)}\right) \\
& \leq\left\|P^{-T}\right\|_{L_{2}(\Omega)}^{2}|\mathbf{u}|_{H^{1}(\Omega)}|\mathbf{v}|_{H^{1}(\Omega)} .
\end{aligned}
$$

For the second term in (3.2) we consider the inequality $\operatorname{tr}(A) \operatorname{tr}(B) \leq n\|A\|\|B\|$ and obtain

$$
\begin{aligned}
& \int_{\Omega} \operatorname{tr}\left(P^{-1} \nabla \mathbf{u}\right) \operatorname{tr}\left(P^{-1} \nabla \mathbf{v}\right) \mathrm{d} \mathbf{x} \leq \int_{\Omega} 3\left\|P^{-1} \nabla \mathbf{u}\right\|\left\|P^{-1} \nabla \mathbf{v}\right\| \mathrm{d} \mathbf{x} \\
& \leq 3\left\|P^{-1} \nabla \mathbf{u}\right\|_{L_{2}(\Omega)}\left\|P^{-1} \nabla \mathbf{v}\right\|_{L_{2}(\Omega)} \leq 3\left\|P^{-T}\right\|_{L_{2}(\Omega)}^{2}|\mathbf{u}|_{H^{1}(\Omega)}|\mathbf{v}|_{H^{1}(\Omega)} .
\end{aligned}
$$

By combining these two results we obtain

$$
a(\mathbf{u}, \mathbf{v}) \leq c\left\|P^{-T}\right\|_{L_{2}(\Omega)}^{2}|\mathbf{u}|_{H^{1}(\Omega)}|\mathbf{v}|_{H^{1}(\Omega)} \leq c\left\|P^{-T}\right\|_{L_{2}(\Omega)}^{2}\|\mathbf{u}\|_{H^{1}(\Omega)}\|\mathbf{v}\|_{H^{1}(\Omega)} .
$$


Since unique solvability follows from the $\mathbf{H}^{1}$-continuity (3.3) and $\mathbf{H}^{1}$-ellipticity we have to establish both for our bilinear form. Thus, we are left with showing that $a(\cdot, \cdot)$ can be bounded from below by $|\cdot|_{H^{1}(\Omega)}^{2}$. This lower bound can be obtained by a suitable generalized Korn inequality, $c f$. Section 6 , Theorems 6.1 and 6.2 since

$$
a(\mathbf{u}, \mathbf{u})=\mu_{e}\left(\varepsilon_{P}(\mathbf{u}), \varepsilon_{P}(\mathbf{u})\right)_{L_{2}(\Omega)}+\frac{\lambda_{e}}{2}\left(\operatorname{tr}\left(P^{-1} \nabla \mathbf{u}\right), \operatorname{tr}\left(P^{-1} \nabla \mathbf{u}\right)\right)_{L_{2}(\Omega)} \geq \mu_{e}\left(\varepsilon_{P}(\mathbf{u}), \varepsilon_{P}(\mathbf{u})\right)_{L_{2}(\Omega)} .
$$

For our condition number estimate of the FETI-DP method, we need an explicit representation of the elements $\mathbf{r}$ in the null space $\operatorname{ker}\left(\varepsilon_{P}\right)$. We have

$$
\left\|\varepsilon_{P}(\mathbf{r})\right\|^{2}=0 \Leftrightarrow\left\|P^{-1}\left(\nabla \mathbf{r} P^{T}+P \nabla \mathbf{r}^{T}\right) P^{-T}\right\|^{2}=0 \Leftrightarrow \operatorname{sym}\left(\nabla \mathbf{r} P^{T}\right)=0 .
$$

Hence, $\nabla \mathbf{r} P^{T}$ must be a skew symmetric matrix $A(\mathbf{x}) \in \mathfrak{s o}(3):=\left\{X \in \mathbb{R}^{3 \times 3}: X^{T}=-X\right\}$. For such a matrix $A(x)$, we have

$$
\operatorname{tr}(A(\mathbf{x}))=0 \quad \text { and } \quad \nabla \mathbf{r}(\mathbf{x})=A(\mathbf{x}) P^{-T}(\mathbf{x}) .
$$

We use the Curl-operator on both sides of the equation, i.e., with a vector $\mathbf{v}(\mathbf{x}) \in \mathbb{R}^{3}: \operatorname{curl}(\mathbf{v}):=\left(\partial_{2} v_{3}-\right.$ $\left.\partial_{3} v_{2}, \partial_{3} v_{1}-\partial_{1} v_{3}, \partial_{1} v_{2}-\partial_{2} v_{1}\right)^{T}$. Since we have matrices on both sides of the equation, we define the curl of a matrix as the curl of its rows.

If we apply the Curl-operator to the left hand side of the second equation in (3.4), we get the curl of the divergence of a potential in all three rows. Thus, $\operatorname{Curl}(\nabla \mathbf{r})=0$ under the assumption that $\mathbf{r}$ is twice continuously differentiable. We will now apply the curl to the right hand side of the second equality in (3.4). For convenience we introduce $a_{i}(\mathbf{x})$ as the rows of the matrix $A(\mathbf{x})$ and $p_{i}(\mathbf{x})$ as the columns of the matrix $P^{-T}(\mathbf{x})$. We will now calculate the curl of the rows $j \in\{1,2,3\}$ explicitly. Therefore we use the abbreviation $\partial_{k}$ instead of $\frac{\partial}{\partial x_{k}}$ and denote by $\partial_{k} a_{m}$ the component-by-component partial derivative of the row $a_{m}$; an analogous notation is used for the column $p_{m}$

$$
\operatorname{curl}\left[\begin{array}{l}
a_{j} p_{1} \\
a_{j} p_{2} \\
a_{j} p_{3}
\end{array}\right]=\left[\begin{array}{l}
\left(\partial_{2} a_{j}\right) p_{3}-\left(\partial_{3} a_{j}\right) p_{2} \\
\left(\partial_{3} a_{j}\right) p_{1}-\left(\partial_{1} a_{j}\right) p_{3} \\
\left(\partial_{1} a_{j}\right) p_{2}-\left(\partial_{2} a_{j}\right) p_{1}
\end{array}\right]+\left[\begin{array}{l}
a_{j}\left(\partial_{2} p_{3}-\partial_{3} p_{2}\right) \\
a_{j}\left(\partial_{3} p_{1}-\partial_{1} p_{3}\right) \\
a_{j}\left(\partial_{1} p_{2}-\partial_{2} p_{1}\right)
\end{array}\right] .
$$

Here, we dropped the explicit dependence on $\mathbf{x}$. We now denote by $p_{i j}$ the entry in the $i$ th row and the $j$ th column of $P^{-T}$ and obtain

$$
\begin{aligned}
\operatorname{Curl}\left(A P^{-T}\right)= & \underbrace{\left[\begin{array}{lll}
\partial_{1} a_{1} & \partial_{2} a_{1} & \partial_{3} a_{1} \\
\partial_{1} a_{2} & \partial_{2} a_{2} & \partial_{3} a_{2} \\
\partial_{1} a_{3} & \partial_{2} a_{3} & \partial_{3} a_{3}
\end{array}\right]}_{\in M^{3 \times 9}} \cdot \underbrace{\left[\begin{array}{ccc}
0 & -p_{3} & p_{2} \\
p_{3} & 0 & -p_{1} \\
-p_{2} & p_{1} & 0
\end{array}\right]}_{\in M^{9 \times 3}} \\
& +\left[\begin{array}{l}
a_{1} \\
a_{2} \\
a_{3}
\end{array}\right] \cdot\left[\begin{array}{lll}
\partial_{2} p_{13}-\partial_{3} p_{12} & \partial_{3} p_{11}-\partial_{1} p_{13} & \partial_{1} p_{12}-\partial_{2} p_{11} \\
\partial_{2} p_{23}-\partial_{3} p_{22} & \partial_{3} p_{21}-\partial_{1} p_{23} & \partial_{1} p_{22}-\partial_{2} p_{21} \\
\partial_{2} p_{33}-\partial_{3} p_{32} & \partial_{3} p_{31}-\partial_{1} p_{33} & \partial_{1} p_{32}-\partial_{2} p_{31}
\end{array}\right] \\
= & L_{P^{-T}}\left(D_{\mathbf{x}} A\right)+A \cdot \operatorname{Curl}\left(P^{-T}\right) .
\end{aligned}
$$

Here $L_{P^{-T}}\left(D_{\mathbf{x}} A(\mathbf{x})\right)$ denotes the linear operator in $P^{-T}$ applied to the derivative of $A(\mathbf{x})$ defined by the first matrix product. Thus, we have

$$
\operatorname{Curl}(\nabla \mathbf{r}(\mathbf{x}))=\operatorname{Curl}\left(A(\mathbf{x}) P^{-T}(\mathbf{x})\right) \Leftrightarrow 0=L_{P^{-T}}\left(D_{\mathbf{x}} A(\mathbf{x})\right)+A(\mathbf{x}) \operatorname{Curl}\left(P^{-T}(\mathbf{x})\right) .
$$

If we assume that the matrix $P^{-T}$ is a gradient, i.e., there exists a function $\psi: \mathbb{R}^{3} \rightarrow \mathbb{R}^{3}$ such that $P^{-T}(\mathbf{x})=$ $\nabla \psi(\mathbf{x})$, with $\psi$ twice continuously differentiable, it follows that $\operatorname{Curl}\left(P^{-T}(\mathbf{x})\right)=0$. Thus, it is necessary 
that $L_{P^{-T}}\left(D_{\mathbf{x}} A(\mathbf{x})\right)=0$. Since $L_{P^{-T}}$ is a linear operator and invertible if $\operatorname{det}\left(P^{-T}\right) \neq 0$, the condition $L_{P-T}\left(D_{\mathbf{x}} A(\mathbf{x})\right)=0$ is satisfied if and only if $D_{\mathbf{x}} A(\mathbf{x})=0$ which means that $A(\mathbf{x})=$ const. $=\bar{A}$. From this follows

$$
\nabla \mathbf{r}=\bar{A} P^{-T}=\bar{A} \nabla \psi(\mathbf{x}) \quad \Rightarrow \quad \mathbf{r}(\mathbf{x})=\bar{A} \psi(\mathbf{x})+\bar{b},
$$

with a constant translation vector $\bar{b} \in \mathbb{R}^{3}$ and a constant skew-symmetric matrix $\bar{A} \in \mathfrak{s o}$ (3). Thus, we have

$$
\bar{A}=\left[\begin{array}{ccc}
0 & \alpha & -\beta \\
-\alpha & 0 & \gamma \\
\beta & -\gamma & 0
\end{array}\right], \quad \bar{b}=\left[\begin{array}{l}
a \\
b \\
c
\end{array}\right],
$$

with suitable constants $\alpha, \beta, \gamma, a, b, c \in \mathbb{R}$, and we can write $\mathbf{r}(\mathbf{x})$ as

$$
\mathbf{r}(\mathbf{x})=\left[\begin{array}{c}
\alpha \psi^{(2)}(\mathbf{x})-\beta \psi^{(3)}(\mathbf{x})+a \\
-\alpha \psi^{(1)}(\mathbf{x})+\gamma \psi^{(3)}(\mathbf{x})+b \\
\beta \psi^{(1)}(\mathbf{x})-\gamma \psi^{(2)}(\mathbf{x})+c
\end{array}\right]
$$

From this representation we obtain the following basis of $\operatorname{ker}\left(\varepsilon_{P}\right)$

$$
\begin{gathered}
\mathbf{r}_{1}:=\left[\begin{array}{l}
1 \\
0 \\
0
\end{array}\right], \quad \mathbf{r}_{2}:=\left[\begin{array}{l}
0 \\
1 \\
0
\end{array}\right], \quad \mathbf{r}_{3}:=\left[\begin{array}{l}
0 \\
0 \\
1
\end{array}\right], \\
\mathbf{r}_{4}(\mathbf{x}):=\left[\begin{array}{c}
\psi^{(2)}(\mathbf{x}) \\
-\psi^{(1)}(\mathbf{x}) \\
0
\end{array}\right], \quad \mathbf{r}_{5}(\mathbf{x}):=\left[\begin{array}{c}
-\psi^{(3)}(\mathbf{x}) \\
0 \\
\psi^{(1)}(\mathbf{x})
\end{array}\right], \quad \mathbf{r}_{6}(\mathbf{x}):=\left[\begin{array}{c}
0 \\
\psi^{(3)}(\mathbf{x}) \\
-\psi^{(2)}(\mathbf{x})
\end{array}\right] .
\end{gathered}
$$

For the $\mathbf{r}_{l}, l=4,5,6$, we have to consider shifted versions

$$
\begin{array}{r}
\mathbf{r}_{4}(\mathbf{x}):=\frac{1}{H_{\psi}}\left[\begin{array}{c}
\psi^{(2)}(\mathbf{x})-\psi^{(2)}(\hat{\mathbf{x}}) \\
\left.-\psi^{(1)}(\mathbf{x})+\psi^{(1)}(\hat{\mathbf{x}})\right], \\
0
\end{array} \quad \mathbf{r}_{5}(\mathbf{x}):=\frac{1}{H_{\psi}}\left[\begin{array}{c}
-\psi^{(3)}(\mathbf{x})+\psi^{(3)}(\hat{\mathbf{x}}) \\
0 \\
\psi^{(1)}(\mathbf{x})-\psi^{(1)}(\hat{\mathbf{x}})
\end{array}\right],\right. \\
\mathbf{r}_{6}(\mathbf{x}):=\frac{1}{H_{\psi}}\left[\begin{array}{c}
\psi^{(3)}(\mathbf{x})-\psi^{(3)}(\hat{\mathbf{x}}) \\
-\psi^{(2)}(\mathbf{x})+\psi^{(2)}(\hat{\mathbf{x}})
\end{array}\right],
\end{array}
$$

where $H_{\psi}$ is the diameter of the transformed domain $\psi(\Omega)$, i.e., $H_{\psi}:=\operatorname{diam}(\psi(\Omega))$, and $\hat{\mathbf{x}}$ is a shift parameter such that $\psi^{(j)}(\mathbf{x})-\psi^{(j)}(\hat{\mathbf{x}})$ can be estimated by a constant times $H_{\psi}$, i.e., $\left(\psi^{(j)}(\mathbf{x})-\psi^{(j)}(\hat{\mathbf{x}})\right)^{2} \leq C H_{\psi}^{2}$.

Furthermore, we assume that a triangulation $\tau_{h}$ of our domain $\Omega$ is given. The elements $\tau_{h}$ are assumed to be shape regular and to have a typical diameter $h$. We assume that the domain $\Omega$ can be represented exactly as union of tetrahedral finite elements. The corresponding conforming finite element space of piecewise quadratic finite element functions is denoted by $\mathbf{W}^{h}:=\mathbf{W}^{h}(\Omega) \subset \mathbf{H}_{0}^{1}\left(\Omega, \partial \Omega_{D}\right)$. Then we obtain the discrete problem:

Find $\mathbf{u}_{h} \in \mathbf{W}^{h}(\Omega)$ such that

$$
a\left(\mathbf{u}_{h}, \mathbf{v}_{h}\right)=\left(\mathbf{F}, \mathbf{v}_{h}\right)_{L_{2}(\Omega)} \quad \forall \mathbf{v}_{h} \in \mathbf{W}^{h} .
$$

When there is no risk of confusion, we will drop the subscript $h$ in the following sections.

\section{The DUAL-PRimal FETI MEthod}

In this section, we will give an algorithmic description of the dual-primal FETI (Finite Element Tearing and Interconnecting) domain decomposition method for P-elasticity. For related FETI-DP algorithms for linear elasticity problems, see $[21,23,26]$. 
In FETI methods the computational domain is partitioned into non overlapping subdomains, and the continuity of the solution across subdomain boundaries is enforced by Lagrange multipliers. The dual problem is then solved iteratively by a preconditioned Krylov subspace method. As a result, the FETI iterates are in general discontinuous across the subdomain boundaries before convergence.

In dual-primal FETI methods, the variables on the subdomain boundaries are divided into two classes, the primal and the dual variables. As primal variables, labeled with $\Pi$, we refer to variables which are assembled before the iteration and in which continuity is enforced in each iteration step. For dual variables, labeled with $\Delta$, the continuity is established weakly by the introduction of Lagrange multipliers thus enforcing continuity only at convergence. The primal variables also form a globally coupled problem. This global problem is necessary to obtain numerical scalability, i.e., independence on the number of subdomains, but should be kept as small as possible.

\subsection{The basic algorithm}

We assume a Lipschitz domain $\Omega$ partitioned into $N$ subdomains $\Omega_{i}, i=1, \ldots, N$, each of which is the union of finite elements with matching finite element nodes on the boundaries of neighboring subdomains across the interface $\Gamma$. The interface $\Gamma$ is the union of three different groups of open sets, namely, subdomain faces, edges, and vertices. We denote individual faces, edges, and vertices by $\mathcal{F}, \mathcal{E}$, and $\mathcal{V}$, respectively, and follow the presentation given in Klawonn and Rheinbach [21], Section 2; see also Klawonn and Widlund [26] or [31], Section 2.

We will now give an algorithmic description of the basic FETI-DP method. For each subdomain we need the local stiffness matrix $K^{(i)}$, the local load vector $\mathbf{f}^{(i)}$ and the vector of the local nodal values $\mathbf{u}^{(i)}$. The vector of the Lagrange multipliers will be denoted by $\lambda$. We distinguish between interface and interior nodes, the latter denoted by an index $I$, as well as between dual and primal nodes on the interface, denoted by an index $\Delta$ and $\Pi$, respectively. Thus, we have

$$
K^{(i)}=\left[\begin{array}{ccc}
K_{I I}^{(i)} & K_{\Delta I}^{(i) T} & K_{\Pi I}^{(i) T} \\
K_{\Delta I}^{(i)} & K_{\Delta \Delta}^{(i)} & K_{\Pi \Delta}^{(i) T} \\
K_{\Pi I}^{(i)} & K_{\Pi \Delta}^{(i)} & K_{\Pi \Pi}^{(i)}
\end{array}\right], \quad \mathbf{u}^{(i)}=\left[\begin{array}{c}
\mathbf{u}_{I}^{(i)} \\
\mathbf{u}_{\Delta}^{(i)} \\
\mathbf{u}_{\Pi}^{(i)}
\end{array}\right] \quad \text { and } \quad \mathbf{f}^{(i)}=\left[\begin{array}{c}
\mathbf{f}_{I}^{(i)} \\
\mathbf{f}_{\Delta}^{(i)} \\
\mathbf{f}_{\Pi}^{(i)}
\end{array}\right]
$$

Introducing

yields

$$
\mathbf{u}_{B}=\left[\begin{array}{c}
\mathbf{u}_{I} \\
\mathbf{u}_{\Delta}
\end{array}\right], \quad \mathbf{f}_{B}=\left[\begin{array}{c}
\mathbf{f}_{I} \\
\mathbf{f}_{\Delta}
\end{array}\right], \quad \mathbf{u}_{B}^{(i)}=\left[\begin{array}{c}
\mathbf{u}_{I}^{(i)} \\
\mathbf{u}_{\Delta}^{(i)}
\end{array}\right], \quad \mathbf{f}_{B}^{(i)}=\left[\begin{array}{c}
\mathbf{f}_{I}^{(i)} \\
\mathbf{f}_{\Delta}^{(i)}
\end{array}\right]
$$

$$
\begin{aligned}
& K_{B B}=\operatorname{diag}\left(K_{B B}^{(i)}\right) \quad \text { with } \quad K_{B B}^{(i)}=\left[\begin{array}{cc}
K_{I I}^{(i)} & K_{\Delta I}^{(i) T} \\
K_{\Delta I}^{(i)} & K_{\Delta \Delta}^{(i)}
\end{array}\right] \\
& \text { and } \quad K_{\Pi B}=\left[K_{\Pi B}^{(1)}, \ldots, K_{\Pi B}^{(N)}\right] \quad \text { with } \quad K_{\Pi B}^{(i)}=\left[\begin{array}{ll}
K_{\Pi I}^{(i)} & K_{\Pi \Delta}^{(i)}
\end{array}\right] .
\end{aligned}
$$

Next, we assemble the primal variables, indicating the assembled variables by a tilde. This yields

$$
\widetilde{K}=\left[\begin{array}{cc}
K_{B B} & \widetilde{K}_{\Pi B}^{T} \\
\widetilde{K}_{\Pi B} & \widetilde{K}_{\Pi \Pi}
\end{array}\right] \text { with } \widetilde{K}_{\Pi B}=\left[\widetilde{K}_{\Pi B}^{(1)}, \ldots, \widetilde{K}_{\Pi B}^{(N)}\right]
$$

The assembly process can be described using restriction operators $R_{\Pi}^{(i)}$ with

$$
\widetilde{K}_{\Pi B}^{(i)}=R_{\Pi}^{(i) T} K_{\Pi B}^{(i)} \quad \forall i=1, \ldots, N, \quad \widetilde{K}_{\Pi \Pi}=\sum_{i=1}^{N} R_{\Pi}^{(i) T} K_{\Pi \Pi}^{(i)} R_{\Pi}^{(i)} .
$$


The matrices $R_{\Pi}^{(i)}$ only have entries 0 or 1 , the global number of columns equals the number of primal variables, and the number of rows equals the number of primal variables belonging to the subdomain $\Omega_{i}$. The entry in the $i$ th column and the $j$ th row of $R_{\Pi}^{(i)}$ is set to 1 if the $j$ th primal node in the subdomain $\Omega_{i}$ equals the $i$ th primal node in the global problem.

In order to obtain a continuous $\mathbf{u}_{\Delta}$ we introduce a discrete jump operator $B=\left[\begin{array}{ll}0 & B_{\Delta}\end{array}\right]$. The operator $B_{\Delta}$ is constructed with entries $-1,0$, or 1 , in such a way that it will enforce continuity for matching nodes across the interface, i.e., $\mathbf{u}_{B}$ is continuous if $B \mathbf{u}_{B}=0=B_{\Delta} \mathbf{u}_{\Delta}$.

This leads to a new formulation of our problem with $\lambda$ being the vector of the Lagrange multipliers

$$
\left[\begin{array}{ccc}
K_{B B} & \widetilde{K}_{\Pi B}^{T} & B^{T} \\
\widetilde{K}_{\Pi B} & \widetilde{K}_{\Pi \Pi} & 0 \\
B & 0 & 0
\end{array}\right]\left[\begin{array}{c}
\mathbf{u}_{B} \\
\tilde{\mathbf{u}}_{\Pi} \\
\lambda
\end{array}\right]=\left[\begin{array}{c}
\mathbf{f}_{B} \\
\tilde{\mathbf{f}}_{\Pi} \\
\mathbf{0}
\end{array}\right]
$$

In a next step, the variables $\mathbf{u}_{B}$ and $\tilde{\mathbf{u}}_{\Pi}$ are eliminated by block Gaussian elimination which leads to

$$
F \lambda=\mathbf{d},
$$

where

$$
\begin{aligned}
F & =B K_{B B}^{-1} B^{T}+B K_{B B}^{-1} \widetilde{K}_{\Pi B}^{T} \widetilde{S}_{\Pi \Pi}^{-1} \widetilde{K}_{\Pi B} K_{B B}^{-1} B^{T}, \quad \widetilde{S}_{\Pi \Pi}=\widetilde{K}_{\Pi \Pi}-\widetilde{K}_{\Pi B} K_{B B}^{-1} \widetilde{K}_{\Pi B}^{T}, \\
d & =B K_{B B}^{-1} \mathbf{f}_{B}-B K_{B B}^{-1} \widetilde{K}_{\Pi B}^{T} \widetilde{S}_{\Pi \Pi}^{-1}\left(\mathbf{f}_{\Pi}-\widetilde{K}_{\Pi B} K_{B B}^{-1} \mathbf{f}_{B}\right) .
\end{aligned}
$$

Before we are going to construct our preconditioner, we give an alternative representation of $F$ which is used in our convergence analysis in Section 7 . We describe $F$ in terms of the Schur complement $\widetilde{S}_{\varepsilon}$, which we obtain by eliminating only the interior variables in $\widetilde{K}$

$$
\underbrace{\left[\begin{array}{cc}
K_{\Delta \Delta}-K_{\Delta I} K_{I I}^{-1} K_{\Delta I}^{T} & \widetilde{K}_{\Pi \Delta}^{T}-K_{\Delta I} K_{I I}^{-1} \widetilde{K}_{\Pi I}^{T} \\
\widetilde{K}_{\Pi \Delta}-\widetilde{K}_{\Pi I} K_{I I}^{-1} K_{\Delta I}^{T} & \widetilde{K}_{\Pi \Pi}-\widetilde{K}_{\Pi I} K_{I I}^{-1} \widetilde{K}_{\Pi I}^{T}
\end{array}\right]}_{=: \widetilde{S}_{\varepsilon}}\left[\begin{array}{c}
\mathbf{u}_{\Delta} \\
\tilde{\mathbf{u}}_{\Pi}
\end{array}\right]=\left[\begin{array}{l}
\mathbf{f}_{\Delta}-K_{\Delta I} K_{I I}^{-1} \mathbf{f}_{I} \\
\tilde{\mathbf{f}}_{\Pi}-\widetilde{K}_{\Pi I} K_{I I}^{-1} \mathbf{f}_{I}
\end{array}\right] .
$$

To use $\widetilde{S}_{\varepsilon}$ for the definition of $F$, we need another restriction operator $\widetilde{R}_{\Delta \Gamma}$ which restricts partially assembled interface variables to their dual displacement part, i.e., such that $\widetilde{R}_{\Delta \Gamma} \mathbf{u}_{\Gamma}=\mathbf{u}_{\Delta}$ for $\mathbf{u}_{\Gamma}^{T}=\left[\mathbf{u}_{\Delta}^{T} \mathbf{u}_{\Pi}^{T}\right]$. With $B_{\Gamma}:=B_{\Delta} \widetilde{R}_{\Delta \Gamma}$, we have

$$
F=B_{\Gamma} \widetilde{S}_{\varepsilon}^{-1} B_{\Gamma}^{T}
$$

To define the standard FETI-DP Dirichlet preconditioner $M^{-1}$, we introduce a scaled jump operator $B_{D, \Delta}:=$ $\left[B_{D, \Delta}^{(1)}, \ldots, B_{D, \Delta}^{(N)}\right]$. It is constructed by scaling the submatrices of $B_{\Delta}$ as follows. Each row of $B_{\Delta}^{(i)}$ with a nonzero entry corresponding to a Lagrange multiplier connecting a subdomain $\Omega_{i}$ with a neighboring subdomain $\Omega_{j}$ at a point $x \in \partial \Omega_{i, h} \cup \partial \Omega_{j, h}$, is multiplied with the scalar factor

$$
\delta_{j}^{\dagger}(x):=\frac{\left(\mu_{e}^{(j)}\right)^{\gamma}}{\sum_{k \in \mathcal{N}_{x}}\left(\mu_{e}^{(k)}\right)^{\gamma}},
$$

where $\mathcal{N}_{x}:=\left\{i \in\{1, \ldots, N\}: x \in \partial \Omega_{i}\right\}$, and $\gamma \in\left[\frac{1}{2}, \infty\right)$.

Finally, we introduce a block-diagonal Schur complement matrix $S_{\varepsilon}:=\operatorname{diag}\left(S_{\varepsilon}^{(i)}\right)$ with $S_{\varepsilon}^{(i)}$ being the Schur complement which we obtain by eliminating the interior variables from $K^{(i)}$. Then

$$
M^{-1}=B_{D, \Delta} R_{\Delta \Gamma} S_{\varepsilon} R_{\Delta \Gamma}^{T} B_{D, \Delta}^{T}=\sum_{i=1}^{N} B_{D, \Delta}^{(i)} R_{\Delta \Gamma}^{(i)} S_{\varepsilon}^{(i)} R_{\Delta \Gamma}^{(i) T} B_{D, \Delta}^{(i) T}
$$


Here, the $R_{\Delta \Gamma}^{(i)}$ are restriction matrices such that

$$
R_{\Delta \Gamma}^{(i)}\left[\begin{array}{c}
\mathbf{u}_{\Delta}^{(i)} \\
\mathbf{u}_{\Pi}^{(i)}
\end{array}\right]=\mathbf{u}_{\Delta}^{(i)} \quad \text { and } \quad R_{\Delta \Gamma}=\operatorname{diag}_{i=1}^{N}\left(R_{\Delta \Gamma}^{(i)}\right)
$$

The application of the preconditioner $M^{-1}$ to a vector only requires the solution of local Dirichlet problems.

We can also express the preconditioner $M^{-1}$ in terms of $\widetilde{S}_{\varepsilon}$ using a local assembly operator $R^{(i)}$

$$
R^{(i) T}=\left[\begin{array}{cc}
R_{\Delta}^{(i) T} & 0 \\
0 & R_{\Pi}^{(i) T}
\end{array}\right] \text { with } R_{\Delta}^{(i) T} \mathbf{u}_{\Delta}^{(i)}=\left[\begin{array}{c}
\mathbf{v}_{\Delta}^{(1)} \\
\vdots \\
\mathbf{v}_{\Delta}^{(N)}
\end{array}\right] \text { and } \mathbf{v}_{\Delta}^{(i)}:=\left\{\begin{array}{c}
\mathbf{0}_{\Delta}^{(j)}, i \neq j \\
\mathbf{u}_{\Delta}^{(j)}, i=j
\end{array}\right.
$$

cf. Klawonn and Widlund [26], Klawonn et al. [29], and Li and Widlund [35]. This leads to the relationship

$$
\widetilde{S}_{\varepsilon}=\sum_{i=1}^{N} R^{(i) T} S_{\varepsilon}^{(i)} R^{(i)}=R^{T} S_{\varepsilon} R \quad \text { with } \quad R^{T}=\left[R^{(1) T} \ldots R^{(N) T}\right]
$$

Relation (4.5) yields another representation of the preconditioner $M^{-1}$

$$
M^{-1}=B_{D, \Gamma} R^{T} S_{\varepsilon} R B_{D, \Gamma}^{T}=B_{D, \Gamma} \widetilde{S}_{\varepsilon} B_{D, \Gamma}^{T},
$$

with $B_{D, \Gamma}=B_{D, \Delta} \widetilde{R}_{\Delta \Gamma}$. For more detailed information, see, e.g., Klawonn and Widlund [26].

\section{Selection of PRimal Constraints}

In order to obtain a scalable FETI-DP algorithm for $P$-elasticity in three dimensions, we need to select an appropriate number of primal constraints. It is well-known that choosing only vertex constraints, i.e., subassembling only in the vertices of the subdomains, leads to an algorithm which has a condition number estimate of the order of $O(H / h)$; see, e.g., Klawonn et al. [27,28], and Farhat et al. [16]. To improve the algorithms, in addition or instead of the vertex constraints, certain averages and first order moments over edges or faces were introduced as primal constraints for the case of linear elasticity; see Klawonn and Widlund [26], Klawonn and Rheinbach [21] and Farhat et al. [16]. Here, we follow the approach of edge averages; see Klawonn and Widlund [26], and Klawonn and Rheinbach [21] and generalize it to the case of P-elasticity. In order to control the kernel of the subdomain stiffness matrices $K^{(i)}$, we have to control the elements of $\operatorname{ker}\left(\varepsilon_{P}\right)$ and thus we need at least six constraints. As in [21-23,26] for linear elasticity, we will work with edge average constraints of the form

$$
g_{n}\left(\mathbf{w}^{(i)}\right):=\frac{\int_{\mathcal{E}^{i k}} w_{l}^{(i)} \mathrm{d} \mathbf{x}}{\int_{\mathcal{E}^{i k}} 1 \mathrm{~d} \mathbf{x}}, \quad n=1, \ldots, 6 .
$$

These constraints can be interpreted as averages over an edge $\mathcal{E}^{i k}$ of the function $w_{l}^{(i)}, l=1,2,3$, which is the lth component of $\mathbf{w}^{(i)}=\left(w_{1}^{(i)}, w_{2}^{(i)}, w_{3}^{(i)}\right) \in \mathbf{W}^{(i)}$.

Definition 5.1. An edge $\mathcal{E}^{i k}$ is called a primal edge if at least one of its displacement components is provided with a constraint.

Such a constraint belongs to a face $\mathcal{F}^{i j}$ if $\mathcal{E}^{i k}$ is a part of the boundary of this face. To define a fully primal face, we introduce six such constraints which have to be linearly independent on $\operatorname{ker}\left(\varepsilon_{P}\right)$, i.e.,

$$
\forall \mathbf{r} \in \operatorname{ker}\left(\varepsilon_{P}\right) \quad: \quad \sum_{n=1}^{6} g_{n}(\mathbf{r})^{2}=0 \Leftrightarrow \mathbf{r}=0 .
$$


Clearly, this is equivalent to

$$
g_{n}(\mathbf{r})=0 \quad \forall n=1, \ldots, 6 \Leftrightarrow \mathbf{r}=0 .
$$

We can obtain such six functionals by choosing three primal edges which belong to the boundary of the face $\mathcal{F}^{i j}$.

Lemma 5.1. Let $P^{-T}=\nabla \psi$ and $\psi$ be a $\mathcal{C}^{1}$-diffeomorphism with $\operatorname{det}(\nabla \psi)$ being bounded from below and above, i.e., $0<c \leq|\operatorname{det}(\nabla \psi)| \leq C<\infty$. Then, for every subdomain face and for the standard case, cf. Assumption 7.1 in Section 7, we can always find six edge averages of the displacement components that are linearly independent when restricted to the space $\operatorname{ker}\left(\varepsilon_{P}\right)$.

The proof is based on a transformation of $\mathcal{E}^{i k}$ to $\psi\left(\mathcal{E}^{i k}\right)$ which leads to the case of standard linear elasticity. The detailed proof can be found in the technical report [31], Section 3. Thus, we have a set of scalar values $\beta_{m n}$ such that

$$
f_{m}(\mathbf{w})=\sum_{n=1}^{6} \beta_{m n} g_{n}(\mathbf{w}) \quad \forall \mathbf{w} \in \mathbf{W}^{(i)}, \forall m=1, \ldots, 6 .
$$

The construction leads to an alternative basis. For an arbitrary $\mathbf{r} \in \operatorname{ker}\left(\varepsilon_{P}\right)$ and $m=1, \ldots, 6, f_{m}\left(\mathbf{r}_{l}\right)=\delta_{m l}$ implies

$$
0=f_{m}(\mathbf{r})=f_{m}\left(\sum_{l=1}^{6} \alpha_{l} \mathbf{r}_{l}\right)=\sum_{l=1}^{6} \alpha_{l} f_{m}\left(\mathbf{r}_{l}\right)=\sum_{l=1}^{6} \alpha_{l} \delta_{m l}=\alpha_{m} \Rightarrow \mathbf{r}=\sum_{l=1}^{6} \alpha_{l} \mathbf{r}_{l}=0 .
$$

Furthermore, we obtain

$$
\left|g_{m}\left(\mathbf{w}^{(i)}\right)\right|^{2} \leq \frac{\left|\int_{\mathcal{E}^{i k}}\left(w_{l}^{(i)}\right)^{2} \mathrm{~d} \mathbf{x}\right|}{\left|\int_{\mathcal{E}^{i k}}(1)^{2} \mathrm{~d} \mathbf{x}\right|} \leq C H_{i}^{-1}\left\|w_{l}^{(i)}\right\|_{L_{2}\left(\mathcal{E}^{i k}\right)}^{2} .
$$

In the last inequality we have used that the length of $\mathcal{E}^{i k}$ is of the order of $H_{i}$. With Lemma A.3, cf. Appendix, we obtain

$$
\left\|\mathbf{w}^{(i)}\right\|_{L_{2}\left(\mathcal{E}^{i k}\right)}^{2} \leq C\left(1+\log \left(\frac{H_{i}}{h_{i}}\right)\right)\left(\left|\mathbf{w}^{(i)}\right|_{H^{1 / 2}\left(\mathcal{F}^{i j}\right)}^{2}+\frac{1}{H_{i}}\left\|\mathbf{w}^{(i)}\right\|_{L_{2}\left(\mathcal{F}^{i j}\right)}^{2}\right) .
$$

This motivates the following definition of a fully primal face; $c f$. also Klawonn and Widlund [26].

Definition 5.2 (fully primal face). A face $\mathcal{F}^{i j}$ is fully primal if, in the space of primal constraints over $\mathcal{F}^{i j}$, there exists a set $f_{m}, m=1, \ldots, 6$, of linear functionals on $\mathbf{W}^{(i)}$ with the following properties:

(1) $\left|f_{m}\left(\mathbf{w}^{(i)}\right)\right|^{2} \leq C H_{i}^{-1}\left(1+\log \left(\frac{H_{i}}{h_{i}}\right)\right)\left(\left|\mathbf{w}^{(i)}\right|_{H^{1 / 2}\left(\mathcal{F}^{i j}\right)}^{2}+\frac{1}{H_{i}}\left\|\mathbf{w}^{(i)}\right\|_{L_{2}\left(\mathcal{F}^{i j}\right)}^{2}\right)$;

(2) $f_{m}\left(\mathbf{r}_{l}\right)=\delta_{m l} \forall m, l=1, \ldots, 6, \mathbf{r}_{l}$ basis element of $\operatorname{ker}\left(\varepsilon_{P}\right)$.

Let us note that the largest of the constants $C$, over all fully primal faces, enters the final bound of the condition number of the iterative method.

\section{KoRn INEQUALITIES}

In this section, we discuss different Korn inequalities which are needed in our convergence analysis in Section 7.

The results needed can be partly found in Neff [38]. As opposed to [38] we are here also interested in the influence of the structural parameter $P$ on the constants in the estimates. For the proofs, see [31], Section 4. In Neff [38], an upper estimate for the expression

$$
\left\|(\nabla \phi) P^{T}(\mathbf{x})+P(\mathbf{x})(\nabla \phi)^{T}\right\|_{L_{2}(\Omega)}^{2}=\int_{\Omega}\left\|(\nabla \phi) P^{T}(\mathbf{x})+P(\mathbf{x})(\nabla \phi)^{T}\right\|^{2} \mathrm{~d} \mathbf{x}
$$

is derived. Here, we have

$$
\left\|P^{-1} \nabla \mathbf{u}+(\nabla \mathbf{u})^{T} P^{-T}\right\|_{L_{2}(\Omega)}^{2}=\int_{\Omega}\left\|P^{-1} \nabla \mathbf{u}+(\nabla \mathbf{u})^{T} P^{-T}\right\|^{2} \mathrm{~d} \mathbf{x},
$$


which can also be represented as

$$
\left\|P^{-1} \nabla \mathbf{u}+(\nabla \mathbf{u}) P^{-T}\right\|_{L_{2}(\Omega)}^{2}=\left\|P^{-1}\left((\nabla \mathbf{u}) P^{T}+P(\nabla \mathbf{u})^{T}\right) P^{-T}\right\|_{L_{2}(\Omega)}^{2} .
$$

If we are able to ensure that the following norm equivalence holds, $\exists 0<c, C<\infty$ :

$$
c\left\|(\nabla \mathbf{u}) P^{T}+P(\nabla \mathbf{u})^{T}\right\|_{L_{2}(\Omega)} \leq\left\|P^{-1}\left((\nabla \mathbf{u}) P^{T}+P(\nabla \mathbf{u})^{T}\right) P^{-T}\right\|_{L_{2}(\Omega)} \leq C\left\|(\nabla \mathbf{u}) P^{T}+P(\nabla \mathbf{u})^{T}\right\|_{L_{2}(\Omega)}
$$

we can use the estimates given in Neff [38] for (6.1) again for (6.2). We are also interested to know how the constants $c$ and $C$ depend on $P$.

Since we know that the $L_{2}$-norm is submultiplicative we have

$$
\left\|P^{-1}\left((\nabla \mathbf{u}) P^{T}+P(\nabla \mathbf{u})^{T}\right) P^{-T}\right\|_{L_{2}(\Omega)} \leq\left\|P^{-T}\right\|_{L_{2}(\Omega)}^{2}\left\|(\nabla \mathbf{u}) P^{T}+P(\nabla \mathbf{u})^{T}\right\|_{L_{2}(\Omega)} .
$$

To obtain the other estimate we use that the spectral matrix-norm is equivalent to the Frobenius matrix-norm on the space of real, finite dimensional $m \times n$ matrices with $n, m<\infty$. We have

$$
\frac{1}{n}\|N\| \leq\|N\|_{2} \leq\|N\|
$$

Now we derive a lower bound for $\left\|L N L^{T}\right\|_{2}$ with $L:=P^{-1}$ and $N:=(\nabla \mathbf{u}) P^{T}+P(\nabla \mathbf{u})^{T}$. Since $N$ is symmetric we have

$$
\left\|L N L^{T}\right\|_{2}=\sup _{\substack{x \in \mathbb{R}^{3} \\ x \neq 0}}\left|\frac{\left\langle N L^{T} x, L^{T} x\right\rangle_{2}}{\langle x, x\rangle_{2}}\right|=\sup _{\substack{y \in \mathbb{R}^{3} \\ L^{-T} y \neq 0}}\left|\frac{\langle N y, y\rangle_{2}}{\left\langle L^{-T} y, L^{-T} y\right\rangle_{2}}\right| .
$$

Using that $N$ is symmetric, $\left\|L^{-T} y\right\|_{2} \leq\left\|L^{-T}\right\|_{2}\|y\|_{2}$, and the lower estimate of (6.4), we obtain

$$
\sup _{\substack{y \in \mathbb{R}^{3} \\ L^{-T} y \neq 0}}\left|\frac{\langle N y, y\rangle_{2}}{\left\langle L^{-T} y, L^{-T} y\right\rangle_{2}}\right| \geq \frac{1}{\left\|L^{-T}\right\|^{2}} \cdot\|N\|_{2} \geq \frac{1}{n\left\|L^{-T}\right\|^{2}} \cdot\|N\| .
$$

Thus, we obtain the following estimate

$$
\left\|P^{-1}\left((\nabla \mathbf{u}) P^{T}+P(\nabla \mathbf{u})^{T}\right) P^{-T}\right\| \geq \frac{1}{3\left\|P^{-T}\right\|^{2}} \cdot\left\|(\nabla \mathbf{u}) P^{T}+P(\nabla \mathbf{u})^{T}\right\|
$$

Next, we consider

$$
\left\|\varepsilon_{P}(\mathbf{u})\right\|_{L_{2}(\Omega)}^{2}=\left\|P^{-1}\left((\nabla \mathbf{u}) P^{T}+P(\nabla \mathbf{u})^{T}\right) P^{-T}\right\|_{L_{2}(\Omega)}^{2} \geq \int_{\Omega} \frac{1}{9\left\|P^{-T}\right\|^{4}}\left\|(\nabla \mathbf{u}) P^{T}+P(\nabla \mathbf{u})^{T}\right\|^{2} \mathrm{~d} \mathbf{x} .
$$

We also have

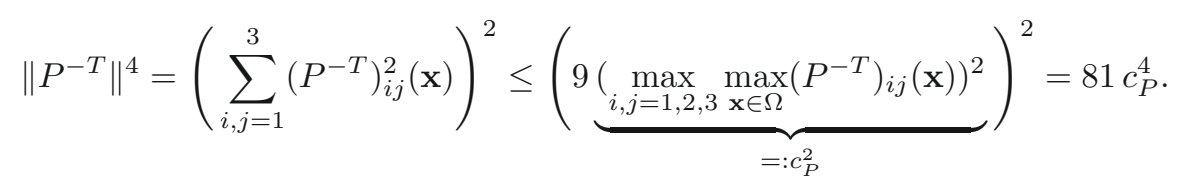


Combining these results with (6.3) and (6.5) leads to the inequality

$$
\begin{aligned}
\frac{1}{27 c_{P}^{2}}\left\|(\nabla \mathbf{u}) P^{T}+P(\nabla \mathbf{u})^{T}\right\|_{L_{2}(\Omega)} & \leq\left(\int_{\Omega}\left(\frac{1}{3\left\|P^{-T}\right\|^{2}}\left\|(\nabla \mathbf{u}) P^{T}+P(\nabla \mathbf{u})^{T}\right\|\right)^{2} \mathrm{~d} \mathbf{x}\right)^{1 / 2} \\
& \leq\left(\int_{\Omega}\left\|P^{-1}\left((\nabla \mathbf{u}) P^{T}+P(\nabla \mathbf{u})^{T}\right) P^{-T}\right\|^{2} \mathrm{~d} \mathbf{x}\right)^{1 / 2} \\
& =\left\|\varepsilon_{P}(\mathbf{u})\right\|_{L_{2}(\Omega)} \\
& \leq\left\|P^{-T}\right\|_{L_{2}(\Omega)}^{2}\left\|(\nabla \mathbf{u}) P^{T}+P(\nabla \mathbf{u})^{T}\right\|_{L_{2}(\Omega)} \\
& \leq 9|\Omega| c_{P}^{2}\left\|(\nabla \mathbf{u}) P^{T}+P(\nabla \mathbf{u})^{T}\right\|_{L_{2}(\Omega)},
\end{aligned}
$$

with $|\Omega|:=\int_{\Omega} 1 \mathrm{~d} \mathbf{x}$.

Let us now consider the Korn inequalities needed in our condition number estimate in Section 7 . Since we work with domain decomposition methods, we may have subdomains $\Omega_{i}$ with zero Dirichlet boundary conditions on part of their boundaries and we can use Korn's first inequality on $\mathbf{H}_{\mathbf{0}}^{\mathbf{1}}\left(\Omega_{i}, \partial \Omega_{D} \cap \partial \Omega_{i}\right)$. But, in general, we also have subdomains with only natural boundary conditions such that we need Korn's second inequality. First we consider the following theorem given in Neff [38], Theorem 4.13.

Theorem 6.1 (generalized Korn's first inequality). Let $\Omega \subset \mathbb{R}^{3}$ be a bounded Lipschitz domain and let $P^{-T}=$ $\nabla \psi \in C^{0}\left(\bar{\Omega}, \mathbb{R}^{3 \times 3}\right) \subset L^{\infty}\left(\bar{\Omega}, \mathbb{R}^{3 \times 3}\right)$ be given with a positive constant $a^{+}$such that $\operatorname{det} P^{T} \geq a^{+}$and let $\psi: \bar{\Omega} \subset$ $\mathbb{R}^{3} \mapsto \mathbb{R}^{3}$ be a $C^{1}$-diffeomorphism. Then there exists a constant $c^{+}>0$ such that

$$
\left\|(\nabla \phi) P^{T}(\mathbf{x})+P(\mathbf{x})(\nabla \phi)^{T}\right\|_{L_{2}(\Omega)}^{2} \geq c^{+}\|\phi\|_{H^{1}(\Omega)}^{2} \quad \forall \phi \in \mathbf{H}_{\mathbf{0}}^{\mathbf{1}}(\Omega, \Gamma)
$$

where $c^{+}:=C(\psi(\Omega), \psi(\Gamma)) \frac{\min _{\mathbf{x} \in \Omega} \operatorname{det}\left(P^{-T}(\mathbf{x})\right)}{\max _{\mathbf{x} \in \Omega} \operatorname{det}\left(P^{-T}(\mathbf{x})\right)} \lambda_{\min , \Omega}\left(P^{T} P\right)$ with $\lambda_{\min , \Omega}\left(P^{T} P\right):=\inf _{\mathbf{x} \in \bar{\Omega}}\left(\lambda_{\min }\left(P^{T} P\right)\right)(\mathbf{x})$.

This theorem combined with the equivalence relation (6.7) leads to

$$
\left\|\varepsilon_{P}(\mathbf{u})\right\|_{L_{2}(\Omega)}^{2} \geq \frac{1}{3^{6} c_{P}^{4}}\left\|(\nabla \mathbf{u}) P^{T}+P(\nabla \mathbf{u})^{T}\right\|_{L_{2}(\Omega)}^{2} \geq \frac{c^{+}}{3^{6} c_{P}^{4}}\|\mathbf{u}\|_{H^{1}(\Omega)}^{2} \geq \frac{c^{+}}{3^{6} c_{P}^{4}}|\mathbf{u}|_{H^{1}(\Omega)}^{2},
$$

for $\mathbf{u} \in \mathbf{H}_{\mathbf{0}}^{\mathbf{1}}(\Omega)$.

The proof can be found in Neff [38] or in our technical report [31], Section 4, where the dependence of $c^{+}$ on $P$ is outlined in detail.

In the case of a subdomain which intersects the Dirichlet boundary with zero boundary conditions we obtain the $H^{1}$-ellipticity of our bilinear form $a(\cdot, \cdot)$ now by using Theorem 6.1 .

Theorem 6.2 (Korn's second inequality). Let us consider the same assumptions as in Theorem 6.1. Then, there exists a constant $c^{+}>0$ such that

$$
\left\|(\nabla \phi) P^{T}(\mathbf{x})+P(\mathbf{x})(\nabla \phi)^{T}\right\|_{L_{2}(\Omega)}^{2}+\|\phi\|_{L_{2}(\Omega)}^{2} \geq c^{+}\|\phi\|_{H^{1}(\Omega)}^{2} \quad \forall \phi \in \mathbf{H}^{\mathbf{1}}(\Omega),
$$

where $c^{+}:=C(\psi(\Omega)) \frac{\min _{\mathbf{x} \in \Omega} \operatorname{det}\left(P^{-T}(\mathbf{x})\right)}{\max _{\mathbf{x} \in \Omega} \operatorname{det}\left(P^{-T}(\mathbf{x})\right)} \min \left\{\lambda_{\min , \Omega}\left(P^{T} P\right), 1\right\}$ with $\lambda_{\min , \Omega}\left(P^{T} P\right):=\inf _{\mathbf{x} \in \bar{\Omega}}\left(\lambda_{\min }\left(P^{T} P\right)\right)(\mathbf{x})$.

As for Theorem 6.1 a detailed proof can be found in the technical report [31], Section 4.

If the subdomain boundary does not intersect the Dirichlet boundary, as in Theorem 6.2, we follow the line of arguments given in Klawonn and Widlund [26]. 
Therefore we introduce two inner products in $\mathbf{H}^{\mathbf{1}}(\Omega)$ for a region of diameter 1:

$$
\begin{aligned}
(\mathbf{u}, \mathbf{v})_{E_{1}} & :=\left(\varepsilon_{P}(\mathbf{u}), \varepsilon_{P}(\mathbf{v})\right)_{L_{2}(\Omega)}+(\mathbf{u}, \mathbf{v})_{L_{2}(\Omega)}, \\
(\mathbf{u}, \mathbf{v})_{E_{2}} & :=\left(\varepsilon_{P}(\mathbf{u}), \varepsilon_{P}(\mathbf{v})\right)_{L_{2}(\Omega)}+\sum_{i=1}^{6}\left(\mathbf{u}, \mathbf{r}_{i}\right)_{L_{2}(\Sigma)}\left(\mathbf{v}, \mathbf{r}_{i}\right)_{L_{2}(\Sigma)}, \quad \text { with } \quad\left(\mathbf{u}, \mathbf{r}_{i}\right)_{L_{2}(\Sigma)}=\int_{\Sigma} \mathbf{u}^{T} \mathbf{r}_{i} \mathrm{~d} x
\end{aligned}
$$

Here, $\Sigma \subset \partial \Omega$ is assumed to have a positive two-dimensional Hausdorff-measure. Obviously, we have:

Lemma 6.1. $\|\cdot\|_{E_{1}}$ and $\|\cdot\|_{E_{2}}$ which we obtain by defining $\|\mathbf{u}\|_{E_{j}}^{2}:=(\mathbf{u}, \mathbf{u})_{E_{j}}$ for $j=1,2$ are norms on $\mathbf{H}^{\mathbf{1}}(\Omega)$.

Lemma 6.2. Let $\Omega \subset \mathbb{R}^{3}$ be a Lipschitz domain of diameter 1 and let $\Sigma \subset \partial \Omega$ be of positive surface measure. Then, there exist constants $0<c \leq C<\infty$ such that

$$
c\|\mathbf{u}\|_{E_{1}} \leq\|\mathbf{u}\|_{E_{2}} \leq C\|\mathbf{u}\|_{E_{1}} \quad \forall \mathbf{u} \in \mathbf{H}^{\mathbf{1}}(\Omega) .
$$

Proof. We first prove the right inequality. Using the Cauchy-Schwarz inequality, Theorem 6.2, and a trace theorem, we obtain

$$
\begin{aligned}
\|\mathbf{u}\|_{E_{2}}^{2} & \leq\left\|\varepsilon_{P}(\mathbf{u})\right\|_{L_{2}(\Omega)}^{2}+\left(\sum_{i=1}^{6}\left(\mathbf{r}_{i}, \mathbf{r}_{i}\right)_{L_{2}(\Sigma)}\right)\|\mathbf{u}\|_{L_{2}(\Sigma)}^{2} \\
& \leq\left\|\varepsilon_{P}(\mathbf{u})\right\|_{L_{2}(\Omega)}^{2}+C(\psi(\Omega))\left(\left\|\varepsilon_{P}(\mathbf{u})\right\|_{L_{2}(\Omega)}^{2}+\|\mathbf{u}\|_{L_{2}(\Omega)}^{2}\right) \leq(1+C(\psi(\Omega)))\|\mathbf{u}\|_{E_{1}}^{2} .
\end{aligned}
$$

To show the left inequality we return to the case of linear elasticity. Therefore we consider that the elements $\mathbf{r} \in \operatorname{ker}\left(\varepsilon_{P}\right)$ are in fact transformed to the elements $\tilde{\mathbf{r}} \in \operatorname{ker}(\varepsilon)$ of linear elasticity, $c f$. proof of Lemma 5.1 in [31], Section 3. We then know from Klawonn and Widlund [26] that

$$
\int_{\xi \in \psi(\Omega)}\left\|\nabla_{\xi} \phi_{e}(\xi)+\left(\nabla_{\xi} \phi_{e}(\xi)\right)^{T}\right\| \mathrm{d} \xi+\sum_{i=1}^{6} \int_{\xi \in \psi(\Sigma)}\left\langle\phi_{e}(\xi), \tilde{\mathbf{r}}_{i}(\xi)\right\rangle^{2} \mathrm{~d} \xi \geq C\left\|\phi_{e}(\xi)\right\|_{E_{1}(\psi(\Omega))}^{2},
$$

where $\phi_{e}(\psi(\mathbf{x})):=\phi_{e}(\xi):=\phi(x)$. The constant $C$ depends on the domains over which we integrate and hence we write $C(\psi(\Omega), \psi(\Sigma))$. This results from Rellich's theorem in the proof in the case of standard linear elasticity and cannot be avoided. The first term on the left hand side of (6.8) can be treated as already done in the proof of Theorem 6.1, i.e.,

$$
\left\|\operatorname{sym}\left(\nabla_{\xi} \phi_{e}(\xi)\right)\right\|_{L_{2}(\psi(\Omega))}^{2} \leq \underbrace{\max _{\mathbf{x} \in \Omega}|\operatorname{det}(\nabla \psi(\mathbf{x}))|}_{=: c_{\operatorname{det}}}\left\|\operatorname{sym}\left(\nabla_{\mathbf{x}} \phi(\mathbf{x}) P^{T}\right)\right\|_{L_{2}(\Omega)}^{2} .
$$

For the second term, for each $i=1, \ldots, 6$, we obtain

$$
\int_{\xi \in \psi(\Sigma)}\left\langle\phi_{e}(\xi), \tilde{\mathbf{r}}_{i}(\xi)\right\rangle^{2} \mathrm{~d} \xi=\int_{\Sigma}\left\langle\phi_{e}(\psi(\mathbf{x})), \tilde{\mathbf{r}}_{i}(\psi(\mathbf{x}))\right\rangle^{2}\|\operatorname{Cof}(\nabla \psi(\mathbf{x})) \cdot \mathbf{n}\| \mathrm{d} \mathbf{x} \leq \underbrace{\max _{\mathbf{x} \in \Omega}\|\operatorname{Cof}(\nabla \psi(\mathbf{x}))\|}_{=: c_{\mathrm{cof}}} \int_{\Omega}\left\langle\phi(\mathbf{x}), \mathbf{r}_{i}(\mathbf{x})\right\rangle^{2} \mathrm{~d} \mathbf{x},
$$

where the cofactor of an invertible matrix is given as $\operatorname{Cof}(A)=\operatorname{det}(A) A^{-T}$. Furthermore, we used Nanson's relation, cf. [20], (2.55). Here, the submultiplicativity and the fact that $\mathbf{n}$ is a unit normal vector are used. Combining these results, we obtain

$$
\begin{aligned}
\|\phi(\mathbf{x})\|_{E_{2}(\Omega)}^{2} & \geq \min \left(\frac{1}{c_{\mathrm{cof}}}, \frac{1}{c_{\mathrm{det}}}\right)\left\|\phi_{e}(\xi)\right\|_{E_{2}(\psi(\Omega))}^{2} \geq C(\psi(\Omega), \psi(\Sigma)) \min \left(\frac{1}{c_{\mathrm{cof}}}, \frac{1}{c_{\mathrm{det}}}\right)\left\|\phi_{e}(\xi)\right\|_{E_{1}(\psi(\Omega))}^{2} \\
& \geq C(\psi(\Omega), \psi(\Sigma)) \min \left(\frac{1}{c_{\mathrm{cof}}}, \frac{1}{c_{\mathrm{det}}}\right) \min _{\mathbf{x} \in \Omega}|\operatorname{det}(\nabla \psi(\mathbf{x}))|\left\|\phi_{e}(\xi)\right\|_{E_{1}(\Omega)}^{2} .
\end{aligned}
$$

The last inequality can be obtained by using the transformation formula. 
Using these results, we obtain the following lemma.

Lemma 6.3. Let $\Omega \subset \mathbb{R}^{3}$ be a Lipschitz domain of diameter 1 , and let $\Sigma \subset \partial \Omega$ be of positive surface measure. Then, there exists a positive constant $C>0$ such that

$$
|\mathbf{u}|_{H^{1}(\Omega)}^{2}+\|\mathbf{u}\|_{L_{2}(\Sigma)}^{2} \leq C\left(\left(\varepsilon_{P}(\mathbf{u}), \varepsilon_{P}(\mathbf{u})\right)_{L_{2}(\Omega)}+\|\mathbf{u}\|_{L_{2}(\Sigma)}^{2}\right) \quad \forall \mathbf{u} \in \mathbf{H}^{\mathbf{1}}(\Omega) .
$$

Proof. By using the standard inequality between norm and seminorm, the expression obtained by Theorem 6.2, and Lemma 6.2, we have

$$
\begin{aligned}
|\mathbf{u}|_{H^{1}(\Omega)}^{2}+\|\mathbf{u}\|_{L_{2}(\Sigma)}^{2} & \leq \frac{1}{c^{+}} \max \left\{3^{6} c_{P}^{4}, 1\right\}\|\mathbf{u}\|_{E_{1}}^{2}+\|\mathbf{u}\|_{L_{2}(\Sigma)}^{2} \\
& \leq \frac{c}{c^{+}} \max \left\{3^{6} c_{P}^{4}, 1\right\}\left(\left(\varepsilon_{P}(\mathbf{u}), \varepsilon_{P}(\mathbf{u})\right)_{L_{2}(\Omega)}+\sum_{i=1}^{6}\left(\mathbf{u}, \mathbf{r}_{i}\right)_{L_{2}(\Sigma)}^{2}\right)+\|\mathbf{u}\|_{L_{2}(\Sigma)}^{2} .
\end{aligned}
$$

By using the Cauchy-Schwarz inequality, we obtain

$$
|\mathbf{u}|_{H^{1}(\Omega)}^{2}+\|\mathbf{u}\|_{L_{2}(\Sigma)}^{2} \leq C_{1}\left(\varepsilon_{P}(\mathbf{u}), \varepsilon_{P}(\mathbf{u})\right)_{L_{2}(\Omega)}+C_{2}\|\mathbf{u}\|_{L_{2}(\Sigma)}^{2} \leq \max \left\{C_{1}, C_{2}\right\}\left(\left(\varepsilon_{P}(\mathbf{u}), \varepsilon_{P}(\mathbf{u})\right)_{L_{2}(\Omega)}+\|\mathbf{u}\|_{L_{2}(\Sigma)}^{2}\right),
$$

where the positive constants $C_{1}, C_{2}$ both depend in different ways on $c_{P}$.

We obtain a new generalized Korn inequality by combining the results obtained so far.

Lemma 6.4. Let $\Omega \subset \mathbb{R}^{3}$ be a Lipschitz domain and let $P^{-T}=\nabla \psi$ with $\nabla \psi \in C^{0}\left(\bar{\Omega}, \mathbb{R}^{3 \times 3}\right) \subset L^{\infty}\left(\bar{\Omega}, \mathbb{R}^{3 \times 3}\right)$ be given with $\operatorname{det} P^{T} \geq a^{+}$and let $\psi: \bar{\Omega} \subset \mathbb{R}^{3} \mapsto \mathbb{R}^{3}$ be a $C^{1}$-diffeomorphism. Then there exist constants $C, c>0$, invariant under dilation, such that

$$
c|\mathbf{u}|_{H^{1}(\Omega)} \leq\left\|\varepsilon_{P}(\mathbf{u})\right\|_{L_{2}(\Omega)} \leq C|\mathbf{u}|_{H^{1}(\Omega)},
$$

where $\mathbf{u} \in\left\{\mathbf{v} \in \mathbf{H}^{\mathbf{1}}(\Omega):(\mathbf{v}, \mathbf{r})_{L_{2}(\Sigma)}=0 \forall \mathbf{r} \in \operatorname{ker}\left(\varepsilon_{P}\right)\right\}$.

Proof. The right inequality was proven in Section 3. There it was shown that

$$
\left\|\varepsilon_{P}(\mathbf{u})\right\|_{L_{2}(\Omega)} \leq c \cdot c_{P}|\mathbf{u}|_{H^{1}(\Omega)} .
$$

There remains to prove the left inequality. We obtain

$$
\left\|\varepsilon_{P}(\mathbf{u})\right\|_{L_{2}(\Omega)}^{2}=\left\|\varepsilon_{P}(\mathbf{u})\right\|_{L_{2}(\Omega)}^{2}+\sum_{i=1}^{6}\left(\mathbf{u}, \mathbf{r}_{i}\right)_{L_{2}(\Sigma)}^{2} \geq c\left(\left\|\varepsilon_{P}(\mathbf{u})\right\|_{L_{2}(\Omega)}^{2}+\|\mathbf{u}\|_{L_{2}(\Omega)}^{2}\right) \geq c^{+} \min \left\{\frac{1}{3^{6} c_{P}^{4}}, 1\right\}|\mathbf{u}|_{H^{1}(\Omega)}^{2} .
$$

Here, we used that $\left(\mathbf{u}, \mathbf{r}_{i}\right)_{L_{2}(\Sigma)}=0$ for all $i=1, \ldots, 6$, as well as Lemma 6.2 and Theorem 6.2. The invariance under dilation can easily be seen by using the transformation formula for a dilation of a domain with diameter $H$.

At this point, we have completed our proof of the equivalence of the norms in Section 3 not only for $\mathbf{u} \in \mathbf{H}_{\mathbf{0}}^{\mathbf{1}}(\Omega)$ but also for $\mathbf{u} \in\left\{\mathbf{v} \in \mathbf{H}^{\mathbf{1}}(\Omega):(\mathbf{v}, \mathbf{r})_{L_{2}(\Sigma)}=0 \forall \mathbf{r} \in \operatorname{ker}\left(\varepsilon_{P}\right)\right\}$.

In the following, we will make extensive use of trace spaces equipped with trace norms. We will recall some definitions in the scalar-valued case which can be extended to the three dimensional case by summing over the components. So let $\Sigma$ be a subset of $\partial \Omega$ with positive measure as before. The norms on the Sobolev spaces 
$H^{1 / 2}(\Sigma)$ and $\mathbf{H}^{\mathbf{1 / 2}}(\partial \Omega):=\left(H^{1 / 2}(\partial \Omega)\right)^{3}$ can be defined as:

$$
\begin{aligned}
|u|_{H^{1 / 2}(\partial \Omega)} & :=\inf _{\substack{v \in H^{1}(\Omega) \\
v \mid \partial \Omega=u}}|v|_{H^{1}(\Omega)} \quad \text { for } \quad u \in H^{1 / 2}(\partial \Omega), \\
\text { and }|\mathbf{u}|_{H^{1 / 2}(\partial \Omega)}^{2} & :=\sum_{i=1}^{3}\left|u_{i}\right|_{H^{1 / 2}(\partial \Omega)}^{2} \text { for } \quad \mathbf{u} \in \mathbf{H}^{\mathbf{1 / 2}}(\partial \Omega) .
\end{aligned}
$$

Another useful seminorm on $\mathbf{H}^{\mathbf{1 / 2}}(\partial \Omega)$ is given by $|\mathbf{u}|_{E_{P}(\partial \Omega)}^{2}:=\inf _{\substack{\mathbf{v} \in \mathbf{H}^{1}(\Omega) \\ \mathbf{v} \mid \partial \Omega=\mathbf{u}}}\left\|\varepsilon_{P}(\mathbf{u})\right\|_{L_{2}(\Omega)}^{2}$. These seminorms lead us to the definitions of the harmonic and $P$-elastic extensions of a function $\mathbf{u} \in \mathbf{H}^{\mathbf{1 / 2}}(\partial \Omega)$ denoted by $\left(\mathbf{u}_{\text {harm }}\right)$ and $\left(\mathbf{u}_{P \text {-elast }}\right)$. These extensions belong to the space $\left\{\mathbf{v} \in \mathbf{H}^{\mathbf{1}}(\Omega):\left.\mathbf{v}\right|_{\partial \Omega}=\mathbf{u}\right\}$ and are defined as

$$
\left|\mathbf{u}_{\text {harm }}\right|_{H^{1}(\Omega)}=|\mathbf{u}|_{H^{1 / 2}(\partial \Omega)} \text { and }\left\|\varepsilon_{P}\left(\mathbf{u}_{P \text {-elast }}\right)\right\|_{L_{2}(\Omega)}=|\mathbf{u}|_{E_{P}(\partial \Omega)} .
$$

Note that the harmonic and elastic extensions minimize the energies defined by the seminorms.

By using Lemma 6.4 and the fact that the $H^{1 / 2}$-seminorm of a function $\mathbf{u}$ is smaller than the $H^{1}$-seminorm of any function which equals $\mathbf{u}$ on $\partial \Omega$, e.g., $\mathbf{u}_{P \text {-elast }}$, we get for $\mathbf{u} \in \mathbf{H}^{\mathbf{1 / 2}}(\partial \Omega)$

$$
|\mathbf{u}|_{H^{1 / 2}(\partial \Omega)}^{2}+\|\mathbf{u}\|_{L_{2}(\partial \Omega)}^{2} \leq C\left\|\varepsilon_{P}\left(\mathbf{u}_{P \text {-elast }}\right)\right\|_{L_{2}(\Omega)}^{2}+\|\mathbf{u}\|_{L_{2}(\partial \Omega)}^{2}=\max \{C, 1\}\left(|\mathbf{u}|_{E_{P}(\partial \Omega)}^{2}+\|\mathbf{u}\|_{L_{2}(\partial \Omega)}^{2}\right) .
$$

Together with a standard scaling argument, we also have two inequalities similar to the Korn inequalities on the trace space $\mathbf{H}^{\mathbf{1} / \mathbf{2}}(\partial \Omega)$.

Lemma 6.5. Let $\Omega \subset \mathbb{R}^{3}$ be a Lipschitz domain of diameter $H$ and $\Sigma \subset \partial \Omega$ an open subset with positive surface measure. Then there exists a constant $C>0$, invariant under dilation, such that

$$
|\mathbf{u}|_{H^{1 / 2}(\Sigma)}^{2}+\frac{1}{H}\|\mathbf{u}\|_{L_{2}(\Sigma)}^{2} \leq C\left(|\mathbf{u}|_{E_{P}(\Sigma)}^{2}+\frac{1}{H}\|\mathbf{u}\|_{L_{2}(\Sigma)}^{2}\right)
$$

where $\mathbf{u} \in \mathbf{H}^{\mathbf{1 / 2}}(\Sigma)$.

We also have an additional Korn inequality.

Lemma 6.6. Let $\Omega \subset \mathbb{R}^{3}$ be a Lipschitz domain of diameter $H$. Furthermore, let $P^{-T}=\nabla \psi \in C^{0}\left(\bar{\Omega}, \mathbb{R}^{3 \times 3}\right) \subset$ $L^{\infty}\left(\bar{\Omega}, \mathbb{R}^{3 \times 3}\right)$ be given with $\operatorname{det} P^{T} \geq a^{+}$and let $\psi: \bar{\Omega} \subset \mathbb{R}^{3} \mapsto \mathbb{R}^{3}$ be a $C^{1}$-diffeomorphism. Then there exists a positive constant $C$, independent of $H$, such that

$$
\inf _{\mathbf{r} \in \mathbf{k e r}\left(\varepsilon_{P}\right)}\|\mathbf{u}-\mathbf{r}\|_{L_{2}(\partial \Omega)}^{2} \leq C H|\mathbf{u}|_{E_{P}(\partial \Omega)}^{2} \quad \forall \mathbf{u} \in \mathbf{H}^{\mathbf{1 / 2}}(\partial \Omega) .
$$

Proof. We can prove the lemma for a domain $\Omega$ of unit diameter and then extend it to a domain with diameter $H$ by a standard scaling argument.

Let $\mathbf{u} \in \mathbf{H}^{\mathbf{1 / 2}}(\partial \Omega)$ be arbitrary but fixed, and let $\mathbf{r} \in \operatorname{ker}\left(\varepsilon_{P}\right)$ be the minimizing element for which $\left(\mathbf{u}-\mathbf{r}, \mathbf{r}_{i}\right)_{L_{2}(\partial \Omega)}=0, i=1, \ldots, 6$. From a standard trace theorem with the $P$-elastic extension we get

$$
\begin{aligned}
\|\mathbf{u}-\mathbf{r}\|_{L_{2}(\partial \Omega)}^{2} & \leq C\left(\left|(\mathbf{u}-\mathbf{r})_{P \text {-elast }}\right|_{H^{1}(\Omega)}^{2}+\left\|(\mathbf{u}-\mathbf{r})_{P \text {-elast }}\right\|_{L_{2}(\Omega)}^{2}\right) \\
& \leq C\left(|\mathbf{u}-\mathbf{r}|_{E_{P}(\partial \Omega)}^{2}+\sum_{i=1}^{6}\left(\mathbf{u}-\mathbf{r}, \mathbf{r}_{i}\right)_{L_{2}(\partial \Omega)}^{2}\right)=C|\mathbf{u}-\mathbf{r}|_{E_{P}(\partial \Omega)}^{2},
\end{aligned}
$$


by using Lemma 6.2 and the second Korn inequality, $c f$. Theorem 6.2. We also have

$$
\begin{gathered}
|\mathbf{u}-\mathbf{r}|_{E_{P}(\partial \Omega)}=\left\|\varepsilon_{P}(\mathbf{u}-\mathbf{r})\right\|_{L_{2}(\Omega)}=\left\|\varepsilon_{P}(\mathbf{u})-\varepsilon_{P}(\mathbf{r})\right\|_{L_{2}(\Omega)}=\left\|\varepsilon_{P}(\mathbf{u})\right\|_{L_{2}(\Omega)}=|\mathbf{u}|_{E_{P}(\partial \Omega)} \\
\Rightarrow\|\mathbf{u}-\mathbf{r}\|_{L_{2}(\partial \Omega)}^{2} \leq C|\mathbf{u}|_{E_{P}(\partial \Omega)}^{2} .
\end{gathered}
$$

Since we use Theorem 6.2, the constant depends on $P$.

In our convergence analysis in Section 7 , we consider in parts the norm which arises if we scale our norm with a matrix $S$, where $S$ is the Schur complement which we obtain from the discretization of a vector-valued Laplace operator scaled by $\mu_{e}:=\max _{i} \mu_{e}^{(i)}$. As in the case of $P$-elasticity, we get local Schur complements $S_{\varepsilon}^{(i)}$ and $S^{(i)}$ by eliminating the interior variables. Since $S$ is block-diagonal with blocks $S^{(i)}$, we work with the norm $|\mathbf{u}|_{S}^{2}:=\sum_{i=1}^{N}\left|u^{(i)}\right|_{S^{(i)}}^{2}$, where $\left|u^{(i)}\right|_{S^{(i)}}^{2}:=\left\langle S^{(i)} u^{(i)}, u^{(i)}\right\rangle$.

A proof of the equivalence of the $S^{(i)}$ - and the $H^{1 / 2}\left(\partial \Omega_{i}\right)$-seminorms of elements of $W^{(i)}$ and for floating subdomains $\Omega_{i}$ can be found already in [8] for the case of piecewise linear elements in two dimensions, and the tools necessary to extend this result to more general finite elements are provided in [58]; see also [55], Section 4.4. In our case, we of course have to multiply $\left|u^{(i)}\right|_{H^{1 / 2}\left(\partial \Omega_{i}\right)}^{2}$ by the factor $\mu_{e}^{(i)}$. The extension to boundary subdomains is also immediate.

This means we have to consider the relation between $S$ and $S_{\varepsilon}$. Since we consider in the basic assumption, cf. Assumption 7.1, that the values $\mu_{e}^{(i)}$ and $\lambda_{e}^{(i)}$ are constant on the subdomains we can consider the norm scaled by $\mu_{e}$ and obtain

$$
|\mathbf{u}|_{S_{\varepsilon}}^{2} \leq 9 c_{P}^{2} \max _{i}\left(1+\frac{\lambda_{e}^{(i)}}{\mu_{e}}\right)|\mathbf{u}|_{S}^{2} \quad \forall \mathbf{w} \in \mathbf{W}^{h}
$$

To complete our notations, we introduce for $\mathbf{u} \in \widetilde{\mathbf{W}}$ a norm $|\mathbf{u}|_{\widetilde{S}_{\varepsilon}}:=\left\langle\widetilde{S}_{\varepsilon} \mathbf{u}, \mathbf{u}\right\rangle^{1 / 2}$. For $\mathbf{u} \in \widetilde{\mathbf{W}}$ and $R \mathbf{u} \in \mathbf{W}$ we get, by using (4.5), the relation

$$
|\mathbf{u}|_{\widetilde{S}_{\varepsilon}}=|R \mathbf{u}|_{S_{\varepsilon}}
$$

\section{Convergence Analysis}

In this section, we provide an analysis of the convergence of our FETI-DP algorithms. We first present an abstract theoretical framework that almost exclusively uses algebraic arguments except for one condition, which requires the analytic tools of Sections 6 and the Appendix A. Then we establish this condition for a special configuration of primal constraints.

We first review the abstract theory developed in Klawonn and Widlund [26], which provides a condition number estimate for the preconditioned FETI-DP matrix $M^{-1} F$. We will work with representations of $F$ and $M^{-1}$ given in (4.2) and (4.6), respectively. We note that the proof of Lemma 7.2 is new.

As indicated before, we let $\mathbf{V}:=$ range $\left(M^{-1}\right) \subset \operatorname{range}\left(B_{D, \Gamma}\right)$ be the space of Lagrange multipliers. If we choose the initial guess $\lambda^{(0)}$ in the conjugate gradient algorithm in $\mathbf{V}$, e.g., $\lambda^{(0)}=0$, then all iterates $\lambda^{(k)}$ will remain in $\mathbf{V}$. As in [25], Section 5, we introduce a projection

$$
P_{D}: \widetilde{\mathbf{W}} \longrightarrow \widetilde{\mathbf{W}}, \quad P_{D}:=B_{D, \Gamma}^{T} B_{\Gamma} .
$$

A simple computation shows that $P_{D}$ preserves the jump of any function $\mathbf{u} \in \widetilde{\mathbf{W}}$ with respect to the jump operator $B_{\Gamma}$, i.e., $B_{\Gamma} P_{D} \mathbf{u}=B_{\Gamma} \mathbf{u}$. Similarly, the transpose $P_{D}^{T}$ preserves the scaled jump, i.e., $B_{D, \Gamma} P_{D}^{T} \mathbf{u}=$ $B_{D, \Gamma} \mathbf{u}$.

Since the elements of $\widehat{\mathbf{W}}$ take common values across the interface, we have $P_{D} \mathbf{u}=0$ for all $\mathbf{u} \in \widehat{\mathbf{W}}$. 
Let $\mathbf{w} \in \widetilde{\mathbf{W}}$, we then have

$$
\left(R^{(i)} P_{D} \mathbf{w}\right)(\mathbf{x})=\sum_{j \in \mathcal{N}_{\mathbf{x}}} \delta_{j}^{\dagger}\left(\left(R^{(i)} \mathbf{w}\right)(\mathbf{x})-\left(R^{(j)} \mathbf{w}\right)(\mathbf{x})\right), \quad \mathbf{x} \in \partial \Omega_{i, h} \cap \Gamma_{h} ;
$$

see [26], (8.3), and [25], (4.4). Here, $\mathcal{N}_{\mathbf{x}}:=\left\{j \in\{1, \ldots, N\}: \mathbf{x} \in \partial \Omega_{j, h}\right\}$ denotes the set of the indices of the subdomains which have the node $\mathbf{x}$ on their boundary and $\delta_{j}^{\dagger}$ is the scalar factor introduced in (4.3). We note that this formula is independent of the particular choice of $B_{\Gamma}$.

To show our condition number estimate, we require the operator $P_{D}$ to satisfy the following stability condition.

Condition 7.1. For all $\mathbf{w} \in \widetilde{\mathbf{W}}$, we have

$$
\left|P_{D} \mathbf{w}\right|_{\widetilde{S}_{\varepsilon}}^{2} \leq C\left(1+\log \left(\frac{H}{h}\right)\right)^{2}|\mathbf{w}|_{\widetilde{S}_{\varepsilon}}^{2}
$$

with $\frac{H}{h}:=\max _{i} \frac{H_{i}}{h_{i}}, H_{i}$ being the subdomain diameter of and $h_{i}$ the typical element diameter in the subdomain $\Omega_{i}$.

This condition will be shown for a particular set of primal variables in this section. When this condition holds for a set of primal constraints we have our condition number estimate; see Klawonn and Widlund [26], Theorem 8.2, for a proof.

Theorem 7.1. Under the assumption that Condition 7.1 holds, the condition number satisfies

$$
\kappa\left(M^{-1} F\right) \leq C\left(1+\log \left(\frac{H}{h}\right)\right)^{2} .
$$

Here, $C$ is independent of $h, H, \gamma$ and the values of $\mu_{e}$, and $\lambda_{e}$ but it depends on $P^{-T}=\nabla \psi$.

We will now give a proof of the condition number estimate, i.e., of Condition 7.1. We follow the structure of the proof in Klawonn and Widlund [26]. We will give the full details for a special case, see [26], Section 8.1, and Assumption 7.2. The other cases considered in Klawonn and Widlund [26], Sections 8.3 and 8.4, can be treated analogously.

The structure of the proof follows Klawonn and Widlund [26]. Here, Condition 7.1 will be established under the following assumptions

Assumption 7.1. (1) Each subdomain $\Omega_{i}$ is the union of a number of shape regular tetrahedral coarse elements, the number of which is uniformly bounded, and all the edges of $\Omega_{i}$ are straight line segments.

(2) Each face has a boundary that is a closed curve formed by at least three edges except when a part of the boundary of the face belongs to $\partial \Omega_{D}$. In the latter case the part of the boundary that belongs to the interface $\Gamma_{h}$ is the union of edges and vertices. We will refer to them as the standard and the Dirichlet case, respectively.

(3) The Lamé constants $\mu_{e}$ and $\lambda_{e}$ do not vary inside one subdomain, and the triangulation of each subdomain is quasi-uniform.

Assumption 7.2. In the decomposition of $\Omega$ into subdomains, no more than three subdomains are common to any edge and with each of the three subdomains sharing a face with the other two. Furthermore, all subdomain vertices are primal and all faces are fully primal; cf. Definition 5.2.

Considering Assumption 7.2, we know that each face $\mathcal{F}^{i j}$ which is common to two subdomains $\Omega_{i}$ and $\Omega_{j}$ has six linear functionals $f_{m}(\cdot)$ which satisfy the conditions of Definition 5.2. In addition, for all $\mathbf{w} \in \widetilde{\mathbf{W}}$, the $f_{m}$ share the same values on the face $\mathcal{F}^{i j}$, i.e.,

$$
f_{m}\left(\mathbf{w}^{(i)}\right)=f_{m}\left(\mathbf{w}^{(j)}\right) \quad \text { where } \mathbf{w}^{(i)}=R^{(i)} \mathbf{w}, \quad \mathbf{w}^{(j)}=R^{(j)} \mathbf{w} .
$$

With these assumptions we can prove Condition 7.1; see Lemma 7.2. 
In order to obtain our estimate, we need a relation between the coefficients $\mu_{e}^{(i)}, \mu_{e}^{(k)}$, and the functions $\delta_{k}^{\dagger}$ which can be proven easily, cf. Klawonn and Widlund [26], Lemma 8.4.

Lemma 7.1. For $\gamma \geq \frac{1}{2}$, we have

$$
\mu_{e}^{(i)}\left(\delta_{k}^{\dagger}\right)^{2} \leq \min \left(\mu_{e}^{(i)}, \mu_{e}^{(k)}\right) .
$$

With this we can prove that Condition 7.1 is satisfied.

Lemma 7.2. Given Assumptions 7.1 and 7.2 , we have for all $\mathbf{w} \in \widetilde{\mathbf{W}}$,

$$
\left|P_{D} \mathbf{w}\right|_{\tilde{S}_{\varepsilon}}^{2} \leq C\left(1+\log \left(\frac{H}{h}\right)\right)^{2}|\mathbf{w}|_{\tilde{S}_{\varepsilon}}^{2}
$$

Proof. Let $\mathbf{w} \in \widetilde{\mathbf{W}}$ be arbitrary. Considering (6.13), we have

$$
\left|P_{D} \mathbf{w}\right|_{\widetilde{S}_{\varepsilon}}=\left|R P_{D} \mathbf{w}\right|_{S_{\varepsilon}}, \quad|\mathbf{w}|_{\tilde{S}_{\varepsilon}}=|R \mathbf{w}|_{S_{\varepsilon}}
$$

Hence with (6.12) and $\mathbf{v}^{(i)}:=R^{(i)} P_{D} \mathbf{w}$ it is sufficient to show that

$$
\sum_{i=1}^{N}\left|\mathbf{v}^{(i)}\right|_{S^{(i)}}^{2}=\left|R P_{D} \mathbf{w}\right|_{S}^{2} \leq C\left(1+\log \left(\frac{H}{h}\right)\right)^{2}|R \mathbf{w}|_{S_{\varepsilon}}^{2} .
$$

Since $R \mathbf{w}=\left[R^{(1)} \mathbf{w}, \ldots, R^{(N)} \mathbf{w}\right]=\left[\mathbf{w}^{(1)}, \ldots, \mathbf{w}^{(N)}\right] \in \mathbf{W}$ it is sufficient to prove for each $i=1, \ldots, N$

$$
\left|\mathbf{v}^{(i)}\right|_{S^{(i)}}^{2} \leq C\left(1+\log \left(\frac{H}{h}\right)\right)^{2} \sum_{j \in \mathcal{N}_{i}}\left|\mathbf{w}^{(j)}\right|_{S_{\varepsilon}^{(j)}}^{2},
$$

where $\mathcal{N}_{i}$ is the set of the indices of neighboring subdomains of $\Omega_{i}$ including $i$ itself.

To prove the estimate, we introduce partition-of-unity functions $\theta_{\mathcal{F}^{i j}}, \theta_{\mathcal{E}^{i k}}$, and $\theta_{\mathcal{V}^{i l}}$ associated with the decomposition of the interface $\Gamma$ into faces, edges, and vertices, $c f$. the definition in the technical report [31], Section 2. These functions are finite element functions on the decomposition $\tau_{h / 2}$. Here, $\tau_{h / 2}$ denotes the decomposition which is obtained when we split each tetrahedron naturally into eight new tetrahedra by using the midpoints of the edges of the quadratic elements as new vertices. The functions $\theta_{\mathcal{F}^{i j}}, \theta_{\mathcal{E}^{i k}}$, and $\theta_{\mathcal{V}^{i l}}$ are supposed to be piecewise linear finite element functions on $\tau_{h / 2}$ taking the value 1 in each point of the respective sets of interface nodes and vanishing elsewhere, e.g.,

$$
\theta_{\mathcal{F}^{i j}}(x)=\left\{\begin{array}{l}
1 \text { if } x \in \mathcal{F}_{h / 2}^{i j} \\
0 \text { if } x \notin \mathcal{F}_{h / 2}^{i j} .
\end{array}\right.
$$

With these functions, we can write $\mathbf{v}^{(i)}$ as

$$
\mathbf{v}^{(i)}=\sum_{\mathcal{F}^{i j} \subset \partial \Omega_{i}} I^{h}\left(\theta_{\mathcal{F}^{i j}} \mathbf{v}^{(i)}\right)+\sum_{\mathcal{E}^{i k} \subset \partial \Omega_{i}} I^{h}\left(\theta_{\mathcal{E}^{i k}} \mathbf{v}^{(i)}\right)+\sum_{\mathcal{V}^{i l} \in \partial \Omega_{i}} \theta_{\mathcal{V}^{i l}} \mathbf{v}^{(i)}\left(\mathcal{V}^{i l}\right) .
$$

Since all vertices are primal, we see from (7.1) that $\mathbf{v}^{(i)}$ vanishes at all vertices and

$$
\mathbf{v}^{(i)}=\sum_{\mathcal{F}^{i j} \subset \partial \Omega_{i}} I^{h}\left(\theta_{\mathcal{F}^{i j}} \mathbf{v}^{(i)}\right)+\sum_{\mathcal{E}^{i k} \subset \partial \Omega_{i}} I^{h}\left(\theta_{\mathcal{E}^{i k}} \mathbf{v}^{(i)}\right) .
$$


Face terms. Since the faces $\mathcal{F}^{i j}$ are shared by the two subdomains $\Omega_{i}$ and $\Omega_{j}$, there remains only one term in $(7.2)$

$$
I^{h}\left(\theta_{\mathcal{F}^{i j}} \delta_{j}^{\dagger}\left(\mathbf{w}^{(i)}-\mathbf{w}^{(j)}\right)\right)
$$

All faces are chosen to be fully primal and thus we have six linear functionals $f_{m}^{\mathcal{F}^{i j}}(\cdot)=f_{m}(\cdot)$ on $\mathcal{F}^{i j}$ which satisfy $f_{m}^{\mathcal{F}^{i j}}\left(\mathbf{w}^{(i)}\right)=f_{m}^{\mathcal{F}^{i j}}\left(\mathbf{w}^{(j)}\right)$ for $m=1, \ldots, 6$. From Definition 5.2 follows for the basis elements of $\operatorname{ker}\left(\varepsilon_{P}\right)$ that $f_{m}^{\mathcal{F}^{i j}}\left(\mathbf{r}_{n}\right)=\delta_{m n}$ for $m, n=1, \ldots, 6$. Next, we consider

$$
\mathbf{w}^{(i)}-\mathbf{w}^{(j)}=\left(\mathbf{w}^{(i)}-\sum_{m=1}^{6} f_{m}^{\mathcal{F}^{i j}}\left(\mathbf{w}^{(i)}\right) \mathbf{r}_{m}\right)-\left(\mathbf{w}^{(j)}-\sum_{m=1}^{6} f_{m}^{\mathcal{F}^{i j}}\left(\mathbf{w}^{(j)}\right) \mathbf{r}_{m}\right) .
$$

Using the representation of an arbitrary element $\mathbf{r}^{(i)} \in \operatorname{ker} \varepsilon_{P}$, with $\mathbf{r}^{(i)} \in \mathbf{W}^{(i)}$ in terms of the basis $\left(\mathbf{r}_{m}\right)_{m=1, \ldots, 6}$, we obtain

$$
\mathbf{r}^{(i)}=\sum_{m=1}^{6} \alpha_{m} \mathbf{r}_{m}=\sum_{m=1}^{6} f_{m}^{\mathcal{F}^{i j}}\left(\sum_{n=1}^{6} \alpha_{n} \mathbf{r}_{n}\right) \mathbf{r}_{m}=\sum_{m=1}^{6} f_{m}^{\mathcal{F}^{i j}}\left(\mathbf{r}^{(i)}\right) \mathbf{r}_{m}
$$

We extend the first term of the right hand side in (7.3) by using (7.4)

$$
\mathbf{w}^{(i)}-\sum_{m=1}^{6} f_{m}^{\mathcal{F}^{i j}}\left(\mathbf{w}^{(i)}\right) \mathbf{r}_{m}=\left(\mathbf{w}^{(i)}-\mathbf{r}^{(i)}\right)-\sum_{m=1}^{6} f_{m}^{\mathcal{F}^{i j}}\left(\mathbf{w}^{(i)}-\mathbf{r}^{(i)}\right) \mathbf{r}_{m}
$$

We can estimate the first term on the right hand side in (7.5) using Lemmas 6.5 and A.5, cf. Appendix

$$
\left|I^{h}\left(\theta_{\mathcal{F} i j}\left(\mathbf{w}^{(i)}-\mathbf{r}^{(i)}\right)\right)\right|_{H^{1 / 2}\left(\partial \Omega_{i}\right)}^{2} \leq C\left(1+\log \left(\frac{H_{i}}{h_{i}}\right)\right)^{2}\left(\left|\mathbf{w}^{(i)}\right|_{E_{P}\left(\partial \Omega_{i}\right)}^{2}+\frac{1}{H_{i}}\left\|\mathbf{w}^{(i)}-\mathbf{r}^{(i)}\right\|_{L_{2}\left(\partial \Omega_{i}\right)}^{2}\right) .
$$

To estimate the second part in (7.5), we need two auxiliary inequalities. By using Lemma A.1, cf. Appendix, we obtain

$$
\left|I^{h}\left(\theta_{\mathcal{F}^{i j}} \mathbf{r}_{m}\right)\right|_{H^{1 / 2}\left(\partial \Omega_{i}\right)}^{2} \leq C H_{i}\left(1+\log \left(\frac{H_{i}}{h_{i}}\right)\right)
$$

By using Definition 5.2 and Lemma 6.5 we get

$$
\left|f_{m}^{\mathcal{F}^{i j}}\left(\mathbf{w}^{(i)}-\mathbf{r}^{(i)}\right)\right|^{2} \leq C H_{i}^{-1}\left(1+\log \left(\frac{H_{i}}{h_{i}}\right)\right)\left(\left|\mathbf{w}^{(i)}\right|_{E_{P}\left(\partial \Omega_{i}\right)}^{2}+\frac{1}{H_{i}}\left\|\mathbf{w}^{(i)}-\mathbf{r}^{(i)}\right\|_{L_{2}\left(\partial \Omega_{i}\right)}^{2}\right) .
$$

Hence, we have

$$
\left|I^{h}\left(\theta_{\mathcal{F}^{i j}}\left(\sum_{m=1}^{6} f_{m}^{\mathcal{F}^{i j}}\left(\mathbf{w}^{(i)}-\mathbf{r}^{(i)}\right) \mathbf{r}_{m}\right)\right)\right|_{H^{1 / 2}\left(\mathcal{F}^{i j}\right)}^{2} \leq C\left(1+\log \left(\frac{H_{i}}{h_{i}}\right)\right)^{2}\left(\left|\mathbf{w}^{(i)}\right|_{E_{P}\left(\partial \Omega_{i}\right)}^{2}+\frac{1}{H_{i}}\left\|\mathbf{w}^{(i)}-\mathbf{r}^{(i)}\right\|_{L_{2}\left(\partial \Omega_{i}\right)}^{2}\right) .
$$


Combining (7.6) and (7.8) with the triangle inequality from (7.5), we obtain the estimate

$$
\begin{aligned}
& \mu_{e}^{(i)}\left|I^{h}\left(\theta_{\mathcal{F}^{i j}}\left(\mathbf{w}^{(i)}-\sum_{m=1}^{6} f_{m}^{\mathcal{F}^{i j}}\left(\mathbf{w}^{(i)}\right) \mathbf{r}_{m}\right)\right)\right|_{H^{1 / 2}\left(\partial \Omega_{i}\right)}^{2} \\
& =\mu_{e}^{(i)}\left|I^{h}\left(\theta_{\mathcal{F}^{i j}}\left(\left(\mathbf{w}^{(i)}-\mathbf{r}^{(i)}\right)-\sum_{m=1}^{6} f_{m}^{\mathcal{F}^{i j}}\left(\mathbf{w}^{(i)}-\mathbf{r}^{(i)}\right) \mathbf{r}_{m}\right)\right)\right|_{H^{1 / 2}\left(\partial \Omega_{i}\right)}^{2} \\
& \leq 2 \mu_{e}^{(i)}\left|I^{h}\left(\theta_{\mathcal{F}^{i j}}\left(\mathbf{w}^{(i)}-\mathbf{r}^{(i)}\right)\right)\right|_{H^{1 / 2}\left(\partial \Omega_{i}\right)}^{2}+2 \mu_{e}^{(i)}\left|I^{h}\left(\theta_{\mathcal{F}^{i j}}\left(\sum_{m=1}^{6} f_{m}^{\mathcal{F}^{i j}}\left(\mathbf{w}^{(i)}-\mathbf{r}^{(i)}\right) \mathbf{r}_{m}\right)\right)\right|_{H^{1 / 2}\left(\partial \Omega_{i}\right)}^{2} \\
& \leq C\left(1+\log \left(\frac{H_{i}}{h_{i}}\right)\right)^{2} \mu_{e}^{(i)}\left(\left|\mathbf{w}^{(i)}\right|_{E_{P}\left(\partial \Omega_{i}\right)}^{2}+\frac{1}{H_{i}}\left\|\mathbf{w}^{(i)}-\mathbf{r}^{(i)}\right\|_{L_{2}\left(\partial \Omega_{i}\right)}^{2}\right) .
\end{aligned}
$$

Since $\mathbf{r}^{(i)} \in \mathbf{W}^{(i)}$ is arbitrary, we can assume that we have chosen the minimizing $\mathbf{r}^{(i)}$, as in Lemma 6.6, and obtain

This yields

$$
\left\|\mathbf{w}^{(i)}-\mathbf{r}^{(i)}\right\|_{L_{2}\left(\partial \Omega_{i}\right)}^{2} \leq C H_{i}\left|\mathbf{w}^{(i)}\right|_{E_{P}\left(\partial \Omega_{i}\right)}^{2} .
$$

$$
\mu_{e}^{(i)}\left|I^{h}\left(\theta_{\mathcal{F}^{i j}}\left(\mathbf{w}^{(i)}-\sum_{m=1}^{6} f_{m}^{\mathcal{F}^{i j}}\left(\mathbf{w}^{(i)}\right) \mathbf{r}_{m}\right)\right)\right|_{H^{1 / 2}\left(\partial \Omega_{i}\right)}^{2} \leq C\left(1+\log \left(\frac{H_{i}}{h_{i}}\right)\right)^{2} \mu_{e}^{(i)}\left|\mathbf{w}^{(i)}\right|_{E_{P}\left(\partial \Omega_{i}\right)}^{2} .
$$

We can proceed in the same way for the second term of the right hand side in (7.3) and obtain

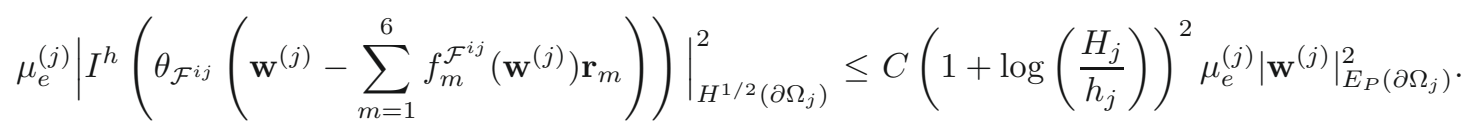

These estimates, together with the triangle inequality, (7.3), and Lemma 7.1, yield

$$
\begin{aligned}
& \mu_{e}^{(i)}\left|I^{h}\left(\theta_{\mathcal{F}^{i j}} \delta_{j}^{\dagger}\left(\mathbf{w}^{(i)}-\mathbf{w}^{(j)}\right)\right)\right|_{H_{00}^{1 / 2}\left(\mathcal{F}^{i j}\right)}^{2} \\
& \leq C\left(1+\log \left(\frac{H_{i}}{h_{i}}\right)\right)^{2} \mu_{e}^{(i)}\left|\mathbf{w}^{(i)}\right|_{E_{P}\left(\partial \Omega_{i}\right)}^{2}+C\left(1+\log \left(\frac{H_{j}}{h_{j}}\right)\right)^{2} \mu_{e}^{(j)}\left|\mathbf{w}^{(j)}\right|_{E_{P}\left(\partial \Omega_{j}\right)}^{2} .
\end{aligned}
$$

Edge terms. Since we assume that at most three subdomains are common to a single edge, $c f$. Assumption 7.2, two subdomains sharing an edge also share a face. Thus, we can reduce our edge estimates to estimates on the corresponding faces using Lemma A.3, cf. Appendix, and the results obtained in this section so far.

From (7.1), we see, by using Lemma A.2, cf. Appendix, that we have to estimate

$$
\mu_{e}^{(i)}\left\|\delta_{j}^{\dagger}\left(\mathbf{w}^{(i)}-\mathbf{w}^{(j)}\right)\right\|_{L_{2}\left(\mathcal{E}^{i k}\right)}^{2}+\mu_{e}^{(i)}\left\|\delta_{k}^{\dagger}\left(\mathbf{w}^{(i)}-\mathbf{w}^{(k)}\right)\right\|_{L_{2}\left(\mathcal{E}^{i k}\right)}^{2} .
$$

The analysis for the first term will be carried out in detail. The second term can then be treated analogously.

Let us assume that the edge $\mathcal{E}^{i k}$ belongs to the boundary of the face $\mathcal{F}^{i j}$ common to $\Omega_{i}$ and $\Omega_{j}$. Using Lemma 7.1, (7.3), and the triangle inequality, we obtain

$$
\begin{aligned}
\mu_{e}^{(i)}\left\|\delta_{j}^{\dagger}\left(\mathbf{w}^{(i)}-\mathbf{w}^{(j)}\right)\right\|_{L_{2}\left(\mathcal{E}^{i k}\right)}^{2} & \leq \min \left(\mu_{e}^{(i)}, \mu_{e}^{(j)}\right)\left\|\mathbf{w}^{(i)}-\mathbf{w}^{(j)}\right\|_{L_{2}\left(\mathcal{E}^{i k}\right)}^{2} \\
& \leq 2 \mu_{e}^{(i)}\left\|\mathbf{w}^{(i)}-\sum_{l=1}^{6} f_{l}^{\mathcal{F}^{i j}}\left(\mathbf{w}^{(i)}\right) \mathbf{r}_{l}\right\|_{L_{2}\left(\mathcal{E}^{i k}\right)}^{2}+2 \mu_{e}^{(j)}\left\|\mathbf{w}^{(j)}-\sum_{l=1}^{6} f_{l}^{\mathcal{F}^{i j}}\left(\mathbf{w}^{(j)}\right) \mathbf{r}_{l}\right\|_{L_{2}\left(\mathcal{E}^{i k}\right)}^{2} .
\end{aligned}
$$


To estimate the first term, we use the identity (7.5) and choose $\mathbf{r}^{(i)} \in \mathbf{W}^{(i)}$ arbitrarily. Combining this with the triangle inequality and Lemma A.3, cf. Appendix, we obtain

$$
\begin{aligned}
2 \mu_{e}^{(i)}\left\|\mathbf{w}^{(i)}-\sum_{l=1}^{6} f_{l}^{\mathcal{F}^{i j}}\left(\mathbf{w}^{(i)}\right) \mathbf{r}_{l}\right\|_{L_{2}\left(\mathcal{E}^{i k}\right)}^{2} \leq & 4 \mu_{e}^{(i)}\left\|\mathbf{w}^{(i)}-\mathbf{r}^{(i)}\right\|_{L_{2}\left(\mathcal{E}^{i k}\right)}^{2}+4 \mu_{e}^{(i)}\left\|\sum_{l=1}^{6} f_{l}^{\mathcal{F}^{i j}}\left(\mathbf{w}^{(i)}-\mathbf{r}^{(i)}\right) \mathbf{r}_{l}\right\|_{L_{2}\left(\mathcal{E}^{i k}\right)}^{2} \\
\leq & C\left(1+\log \left(\frac{H_{i}}{h_{i}}\right)\right) \mu_{e}^{(i)}\left(\left|\mathbf{w}^{(i)}-\mathbf{r}^{(i)}\right|_{H^{1 / 2}\left(\partial \Omega_{i}\right)}^{2}+\frac{1}{H_{i}}\left\|\mathbf{w}^{(i)}-\mathbf{r}^{(i)}\right\|_{L_{2}\left(\partial \Omega_{i}\right)}^{2}\right) \\
& +C \mu_{e}^{(i)} \sum_{l=1}^{6}\left|f_{l}^{\mathcal{F}^{i j}}\left(\mathbf{w}^{(i)}-\mathbf{r}^{(i)}\right)\right|^{2}\left\|\mathbf{r}_{l}\right\|_{L_{2}\left(\mathcal{E}^{i k}\right)}^{2} .
\end{aligned}
$$

Since the length of $\mathcal{E}^{i k}$ is of the order of $\min \left(H_{i}, H_{j}\right)$, it can easily be shown that

$$
\left\|\mathbf{r}_{l}\right\|_{L_{2}\left(\mathcal{E}^{i k}\right)}^{2} \leq C \min \left(H_{i}, H_{j}\right), \quad l=1,2,3,
$$

with a constant $C$ independent of $H, h$ and $\mu_{e}^{(i)}, c f$. [26], (8.14). The shifted basis elements of $\operatorname{ker}\left(\varepsilon_{P}\right), c f .(3.6)$, lead to

Thus, we have

$$
\left\|\mathbf{r}_{l}\right\|_{L_{2}\left(\mathcal{E}^{i k}\right)}^{2} \leq C \int_{\mathcal{E}^{i k}} 1 \mathrm{~d} \mathbf{x}=C\left|\mathcal{E}^{i k}\right| \leq C \min \left(H_{i}, H_{j}\right) .
$$

$$
\left\|\mathbf{r}_{l}\right\|_{L_{2}\left(\mathcal{E}^{i k}\right)}^{2} \leq C \min \left(H_{i}, H_{j}\right), \quad l=1, \ldots, 6 .
$$

We can proceed with all terms obtained so far as before and get

$$
\begin{aligned}
& 2 \mu_{e}^{(i)}\left\|\mathbf{w}^{(i)}-\sum_{l=1}^{6} f_{l}^{\mathcal{F}^{i j}}\left(\mathbf{w}^{(i)}\right) \mathbf{r}_{l}\right\|_{L_{2}\left(\mathcal{E}^{i k}\right)}^{2} \leq C \mu_{e}^{(i)}\left(1+\log \left(\frac{H_{i}}{h_{i}}\right)\right)\left|\mathbf{w}^{(i)}\right|_{E_{P}\left(\partial \Omega_{i}\right)}^{2}, \\
& 2 \mu_{e}^{(j)}\left\|\mathbf{w}^{(j)}-\sum_{l=1}^{6} f_{l}^{\mathcal{F}^{i j}}\left(\mathbf{w}^{(j)}\right) \mathbf{r}_{l}\right\|_{L_{2}\left(\mathcal{E}^{i k}\right)}^{2} \leq C \mu_{e}^{(j)}\left(1+\log \left(\frac{H_{j}}{h_{j}}\right)\right)\left|\mathbf{w}^{(j)}\right|_{E_{P}\left(\partial \Omega_{j}\right)}^{2} .
\end{aligned}
$$

Combining these two results, we have

$$
\mu_{e}^{(i)}\left\|\delta_{j}^{\dagger}\left(\mathbf{w}^{(i)}-\mathbf{w}^{(j)}\right)\right\|_{L_{2}\left(\mathcal{E}^{i k}\right)}^{2} \leq C \mu_{e}^{(i)}\left(1+\log \left(\frac{H_{i}}{h_{i}}\right)\right)\left|\mathbf{w}^{(i)}\right|_{E_{P}\left(\partial \Omega_{i}\right)}^{2}+C \mu_{e}^{(j)}\left(1+\log \left(\frac{H_{j}}{h_{j}}\right)\right)\left|\mathbf{w}^{(j)}\right|_{E_{P}\left(\partial \Omega_{j}\right)}^{2} .
$$

\section{NUMERICAL RESUlts}

In this section we report on a series of computational experiments which are carried out to confirm numerically our theoretical findings. The computations were performed on a compute cluster consisting of 8 dual Opteron processor nodes with $2.2 \mathrm{GHz}$ and $4 \mathrm{~GB}$ memory for each processor. The algorithms were implemented in PETSc [1-3].

All computations are carried out on $\Omega=[0,1]^{3}$ which is discretized in a regular way by first decomposing $\Omega$ into hexahedra which themselves are decomposed into tetrahedra. We first introduce one additional point in the center of the hexahedron which we connect with each vertex of the hexahedron. This results in six pyramids with square bases. By splitting each base into two triangles we obtain 12 tetrahedra for each hexahedron; $c f$. Figure 2. The material parameters are $E=210$ and $\nu=0.29$ which corresponds to $\mu_{e} \approx 81.4$ and $\lambda_{e} \approx 112.4$. 


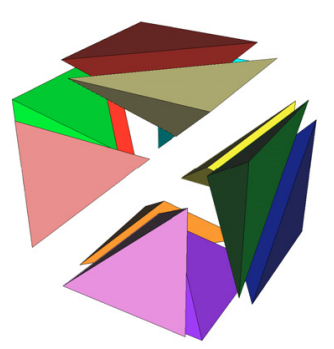

Figure 2. Decomposition of a hexahedron into 12 tetrahedra. (Figure in color available at www. esaim-m2an.org.)

Since we use quadratic elements, additional points on the edges of the tetrahedra are introduced and the number of degrees of freedom for a subdomain can be calculated using $\frac{H}{h}$ by

$$
3\left(\left(2 \cdot \frac{H}{h}\right)^{3}+\left(2 \cdot \frac{H}{h}+1\right)^{3}\right) .
$$

The presentation of our results is divided into three subsections. First, we present results for the case which is completely covered by our analysis, i.e., $P^{-T}=\nabla \psi$ where $\psi: \mathbb{R}^{3} \rightarrow \mathbb{R}^{3}$ is at most piecewise quadratic. The second subsection deals with the case $P^{-T}=\nabla \psi$ when $\psi$ can be an arbitrary differentiable function. In the last subsection, we present results for other cases when $P^{-T}$ is not a gradient but $P$ itself is. Two sets of experiments are carried out. For the first one the subdomain size is kept fixed, i.e., $\frac{H}{h}=$ const., and the number of subdomains, i.e., $\frac{1}{H}$, is increased. According to our theoretical estimate, $c f$. Theorem 7.1, we would expect that the condition number and thus the number of iterations is asymptotically bounded by a constant. In the second set of experiments the number of subdomains is kept fixed, i.e., $\frac{1}{H}=$ const., and the size of the subdomains, i.e., $\frac{H}{h}$, is increased. According to Theorem 7.1, we would expect the number of iterations to grow slowly and the condition number to grow as $O\left(\left(1+\log \left(\frac{H}{h}\right)\right)^{2}\right)$. For our FETI-DP algorithms we consider four different sets of primal variables:

(1) A set with edge average constraints in the interior of the cube.

(2) A set with edge average constraints in the interior and on the Neumann boundary of the cube.

(3) A set with vertex and interior edge average constraints.

(4) A set with vertex constraints and edge average constraints in the interior and on the Neumann boundary.

\subsection{Results for $\boldsymbol{P}^{-T}=\nabla \psi$ where $\psi$ is at most piecewise quadratic}

In this section, we choose $P^{-T}$ as a gradient of an at most piecewise quadratic function. This is the case covered by our theoretical estimates. First, we introduce functions $\psi_{i}: \mathbb{R}^{3} \rightarrow \mathbb{R}^{3}$ which are at most quadratic polynomials in each component $\psi_{i}^{(j)}, j=1,2,3$, then we define $P_{i}^{-T}=\nabla \psi_{i}$. Here all six basis vectors of the kernel of the $P$-elasticity operator, see (3.5), are represented exactly by the finite element basis.

The Dirichlet boundary is chosen to be $\left\{(x, y, z)^{T}=\mathrm{x} \in \mathbb{R}^{3}: z=0\right\}$. To provide the Dirichlet boundary with zero boundary data we choose the initial value of $\varphi$ accordingly. This means that, for $z=0$, we choose $\varphi$ in accordance to the solution if it is known or near the solution if possible. In all other points the initial value for $\varphi$ is the identity, i.e., $\varphi(\mathbf{x})=\mathbf{x}$ if $z \neq 0$. The solution $\varphi$ is analytically known when $P$ itself is also a gradient. Then, the minimal energy will be obtained for $\varphi$ with $P=\nabla \varphi$. If $P$ is not a gradient we do not know the solution in advance. In these cases we either choose Dirichlet boundary values with $P \approx \nabla \varphi$ or $\varphi(\mathbf{x})=\mathbf{x}$. 

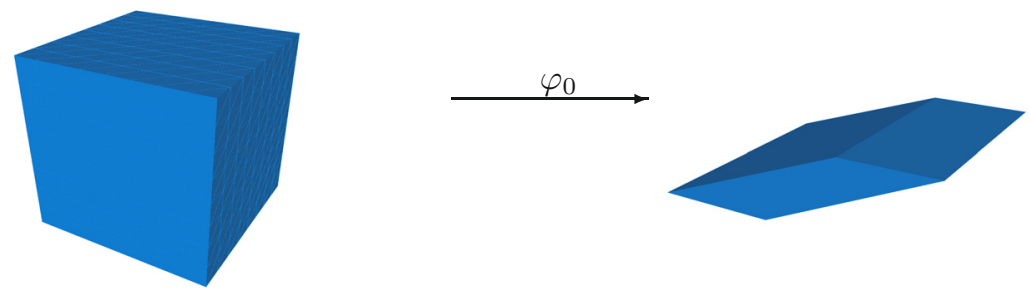

Figure 3. Deformation induced by $P_{0}$.

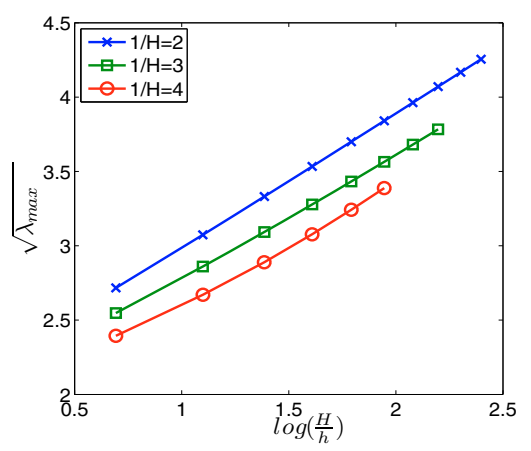

Figure 4. $P^{-T}=\nabla \psi_{0}$, edge constraints without edges on boundary and with vertex constraints.

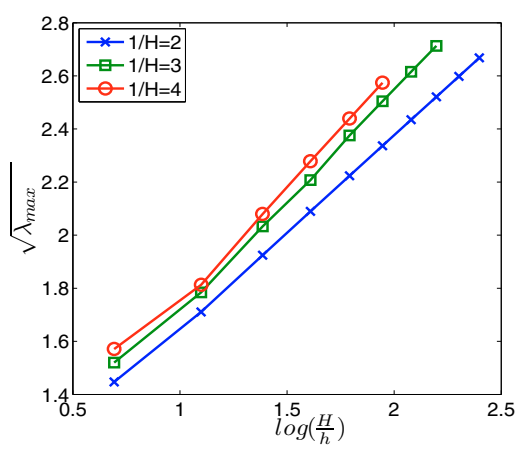

Figure 5. $P^{-T}=\nabla \psi_{0}$, edge constraints with edges on boundary and vertex constraints.

A first example is given by $\psi_{0}(\mathbf{x})=\left(\begin{array}{c}\frac{1}{2} x \\ y \\ 2 x-4 y+4 z\end{array}\right)$, which implies $P_{0}^{-T}=\left(\begin{array}{ccc}\frac{1}{2} & 0 & 0 \\ 0 & 1 & 0 \\ 2 & -4 & 4\end{array}\right)$. Thus, we have $P_{0}=\left(\begin{array}{ccc}2 & 0 & -1 \\ 0 & 1 & 1 \\ 0 & 0 & \frac{1}{4}\end{array}\right)$ and from $P_{0}=\nabla \varphi_{0}$ follows $\varphi_{0}=\left(\begin{array}{c}2 x-z \\ y+z \\ \frac{1}{4} z\end{array}\right) ;$ see also Figure 3

We now perform computations using different sets of primal variables. We use the following notation

c.p.s. = coarse problem size; $\quad \mathrm{N}=$ number of subdomains; $\quad \mathrm{It}=$ iterations;

d.o.f. $=$ degrees of freedom; d.o.f. $/$ dom $=$ d.o.f. per subdomain.

In Tables 1-4 we present the results for $P_{0}^{-T}$ using a fixed subdomain size, i.e., $\frac{1}{H}=$ const. We present the maximum eigenvalue instead of the condition number since the minimum eigenvalue for the preconditioned FETI-DP matrix is, in accordance with the theory, almost exactly 1 in all experiments. The results in Tables 1-4 match our theory, i.e., the condition number and the number of iterations are clearly asymptotically bounded. If we fix the number of subdomains instead and increase the size of the subdomains, i.e., increase $\frac{H}{h}$, see Figures 4 and 5, we obtain straight lines in plots of $\log \left(\frac{H}{h}\right)$ versus $\sqrt{\lambda_{\max }}$. These experiments thus numerically confirm the quadratic-logarithmic dependence on $\frac{H}{h}$.

In fact, for different constant matrices $P$ we always observe condition numbers identical to those in Tables 1-4.

Next, we choose $P^{-T}$ as a linear function, i.e., $P^{-T}$ is the gradient of a function consisting of at most piecewise quadratic polynomials. In these cases $P$ is not necessarily a gradient and therefore we may not know 
TABLE 1. $P^{-T}=\nabla \psi_{0}$, edge constraints without boundary edges.

\begin{tabular}{|c|c|c|c|c|c|c|c|c|c|c|}
\hline \multirow[b]{2}{*}{$N$} & \multirow[b]{2}{*}{ c.p.s. } & \multicolumn{3}{|c|}{$\begin{array}{c}\frac{H}{h}=2 \\
567 \text { d.o.f./dom. }\end{array}$} & \multicolumn{3}{|c|}{$\begin{array}{ll} & \frac{H}{h}=3 \\
1677 & \text { d.o.f./dom. }\end{array}$} & \multicolumn{3}{|c|}{$\begin{array}{cl} & \frac{H}{h}=4 \\
3723 & \text { d.o.f./dom. } \\
\end{array}$} \\
\hline & & d.o.f. & It. & $\lambda_{\max }$ & d.o.f. & It. & $\lambda_{\max }$ & d.o.f. & It. & $\lambda_{\max }$ \\
\hline 27 & 108 & 11775 & 39 & 11.01 & 38073 & 41 & 12.23 & 88347 & 43 & 13.33 \\
\hline 64 & 324 & 27027 & 39 & 9.69 & 88347 & 43 & 10.99 & 206115 & 44 & 12.19 \\
\hline 216 & 1350 & 88347 & 41 & 9.52 & 291927 & 43 & 10.79 & 684723 & 45 & 11.98 \\
\hline 343 & 2268 & 139023 & 40 & 9.51 & 460785 & 43 & 10.77 & 1082427 & 45 & 11.96 \\
\hline 729 & 5184 & 291927 & 39 & 9.51 & 971517 & 43 & 10.76 & & & \\
\hline 1728 & 13068 & 684723 & 39 & 9.51 & & & & & & \\
\hline 2197 & 16848 & 868455 & 40 & 9.51 & & & & & & \\
\hline
\end{tabular}

TABLE 2. $P^{-T}=\nabla \psi_{0}$, edge constraints with boundary.

\begin{tabular}{cc|ccc|ccc|cccc}
\hline & & \multicolumn{3}{|c|}{$\begin{array}{c}H \\
h\end{array}=2$} & \multicolumn{3}{c|}{$\frac{H}{h}=3$} & \multicolumn{4}{c}{$\frac{H}{h}=4$} \\
& & \multicolumn{2}{|c|}{567 d.o.f./dom. } & \multicolumn{2}{c|}{1677} & d.o.f./dom. & \multicolumn{3}{c}{3723} & d.o.f./dom. \\
\hline$N$ & c.p.s. & d.o.f. & It. & $\lambda_{\max }$ & d.o.f. & It. & $\lambda_{\max }$ & d.o.f. & It. & $\lambda_{\max }$ \\
\hline 27 & 288 & 11775 & 23 & 3.40 & 38073 & 26 & 4.41 & 88347 & 29 & 5.27 \\
64 & 684 & 27027 & 23 & 3.57 & 88347 & 27 & 4.66 & 206115 & 30 & 5.57 \\
216 & 2250 & 88347 & 24 & 3.72 & 291927 & 28 & 4.86 & 684723 & 30 & 5.82 \\
343 & 3528 & 139023 & 24 & 3.74 & 460785 & 28 & 4.92 & 1082427 & 31 & 5.88 \\
729 & 7344 & 291927 & 24 & 3.79 & 971517 & 28 & 4.98 & & & \\
1728 & 17028 & 684723 & 24 & 3.81 & & & & & & \\
2197 & 21528 & 868455 & 24 & 3.82 & & & & & & \\
\hline
\end{tabular}

TABLE 3. $P^{-T}=\nabla \psi_{0}$, edge constraints without boundary and with vertex constraints.

\begin{tabular}{cc|ccc|ccc|ccc}
\hline & & \multicolumn{3}{|c|}{$\frac{H}{h}=2$} & \multicolumn{3}{c|}{$\frac{H}{h}=3$} & \multicolumn{3}{c}{$\frac{H}{h}=4$} \\
& & \multicolumn{3}{|c|}{567 d.o.f./dom. } & \multicolumn{2}{c|}{1677 d.o.f./dom. } & \multicolumn{3}{c}{3723 d.o.f./dom. } \\
\hline$N$ & c.p.s. & d.o.f. & It. & $\lambda_{\max }$ & d.o.f. & It. & $\lambda_{\max }$ & d.o.f. & It. & $\lambda_{\max }$ \\
\hline 27 & 192 & 11775 & 29 & 6.49 & 38073 & 33 & 8.18 & 88347 & 36 & 9.56 \\
64 & 540 & 27027 & 30 & 5.73 & 88347 & 34 & 7.13 & 206115 & 37 & 8.35 \\
216 & 2100 & 88347 & 30 & 5.68 & 291927 & 33 & 7.11 & 684723 & 36 & 8.33 \\
343 & 3456 & 139023 & 30 & 5.69 & 460785 & 33 & 7.11 & 1082427 & 36 & 8.33 \\
729 & 7680 & 291927 & 30 & 5.68 & 971517 & 33 & 7.10 & & & \\
1728 & 18876 & 684723 & 30 & 5.68 & & & & & & \\
2197 & 24192 & 868455 & 29 & 5.68 & & & & & & \\
\hline
\end{tabular}

TABLE 4. $P^{-T}=\nabla \psi_{0}$, edge constraints with boundary and vertex constraints.

\begin{tabular}{cc|ccc|ccc|ccc}
\hline & & \multicolumn{3}{|c|}{$\frac{H}{h}=2$} & \multicolumn{3}{c|}{$\frac{H}{h}=3$} & \multicolumn{3}{c}{$\frac{H}{h}=4$} \\
& & \multicolumn{2}{|c|}{567 d.o.f./dom. } & \multicolumn{1}{c|}{1677} & d.o.f./dom. & \multicolumn{3}{c}{3723} & d.o.f./dom. \\
\hline$N$ & c.p.s. & d.o.f. & It. & $\lambda_{\max }$ & d.o.f. & It. & $\lambda_{\max }$ & d.o.f. & It. & $\lambda_{\max }$ \\
\hline 27 & 372 & 11775 & 18 & 2.31 & 38073 & 22 & 3.18 & 88347 & 26 & 4.13 \\
64 & 900 & 27027 & 18 & 2.47 & 88347 & 22 & 3.29 & 206115 & 26 & 4.32 \\
216 & 3000 & 88347 & 19 & 2.59 & 291927 & 22 & 3.39 & 684723 & 26 & 4.48 \\
343 & 4716 & 139023 & 20 & 2.62 & 460785 & 23 & 3.42 & 1082427 & 27 & 4.51 \\
729 & 9840 & 291927 & 19 & 2.66 & 971517 & 23 & 3.44 & & & \\
1728 & 22836 & 684723 & 20 & 2.68 & & & & & & \\
2197 & 28872 & 868455 & 20 & 2.69 & & & & & & \\
\hline
\end{tabular}


TABLE 5. $P^{-T}=\nabla \psi_{1}$, edge constraints without boundary edges.

\begin{tabular}{cc|ccc|ccc|ccc}
\hline & & \multicolumn{3}{|c|}{$\frac{H}{h}=2$} & \multicolumn{3}{c|}{$\frac{H}{h}=3$} & \multicolumn{3}{c}{$\frac{H}{h}=4$} \\
& & \multicolumn{2}{|c|}{567} & d.o.f./dom. & \multicolumn{3}{c|}{1677} & d.o.f./dom. & \multicolumn{3}{c}{3723} & d.o.f./dom. \\
\hline$N$ & c.p.s. & d.o.f. & It. & $\lambda_{\max }$ & d.o.f. & It. & $\lambda_{\max }$ & d.o.f. & It. & $\lambda_{\max }$ \\
\hline 27 & 108 & 11775 & 40 & 13.07 & 38073 & 45 & 14.83 & 88347 & 47 & 16.37 \\
64 & 324 & 27027 & 41 & 11.80 & 88347 & 45 & 13.44 & 206115 & 48 & 14.86 \\
216 & 1350 & 88347 & 41 & 11.03 & 291927 & 45 & 12.54 & 684723 & 47 & 13.86 \\
343 & 2268 & 139023 & 41 & 10.81 & 460785 & 45 & 12.28 & 1082427 & 47 & 13.58 \\
729 & 5184 & 291927 & 41 & 10.50 & 971517 & 45 & 11.92 & & & \\
1728 & 13068 & 684723 & 41 & 10.23 & & & & & & \\
2197 & 16848 & 868455 & 41 & 10.17 & & & & & & \\
\hline
\end{tabular}

TABLE 6. $P^{-T}=\nabla \psi_{1}$, edge constraints without boundary and with vertex constraints.

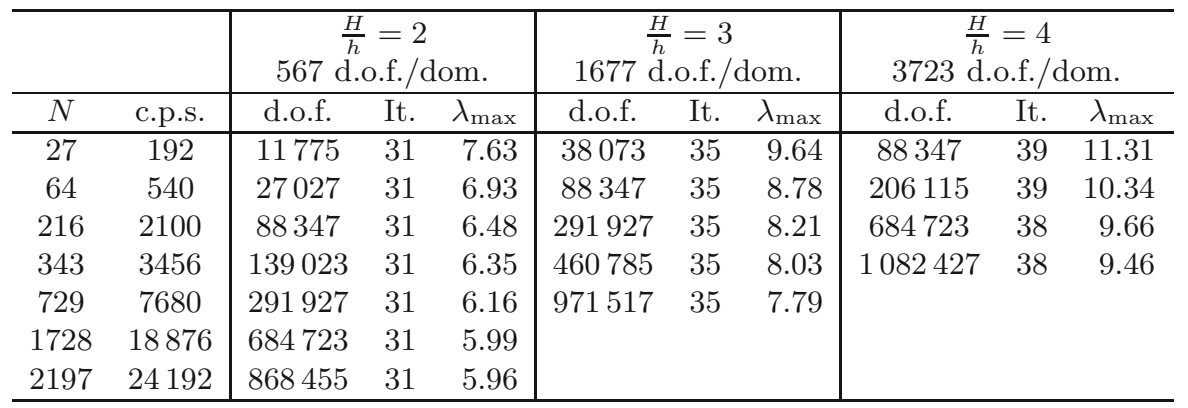

TABLE 7. $P^{-T}=\nabla \psi_{2}$, edge constraints without boundary edges.

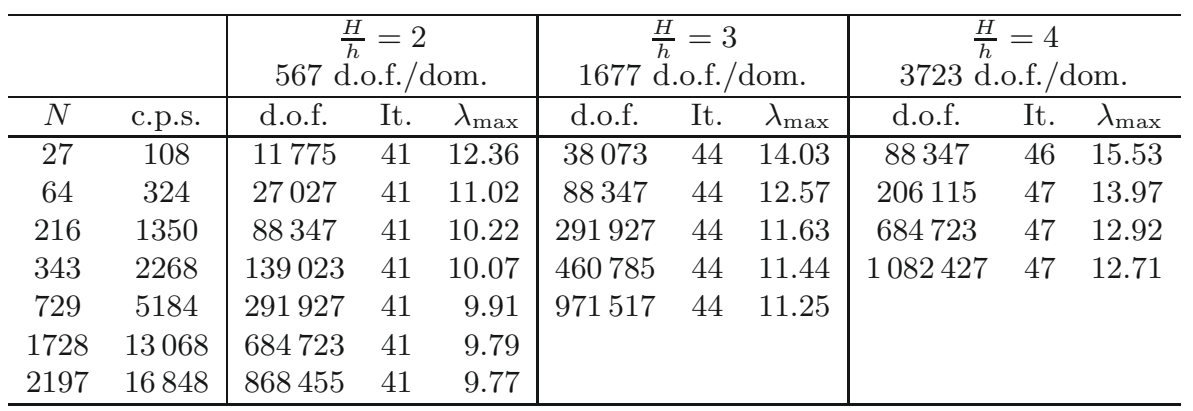

the solution in advance. As examples we consider $\psi_{1}(\mathbf{x})=\left(\begin{array}{c}x^{2}-2 y+3 z \\ x-y^{2}-\frac{1}{2} z \\ -x-y+\frac{1}{2} z^{2}\end{array}\right)$ with $P_{1}^{-T}=\left(\begin{array}{ccc}2 x & -2 & 3 \\ 1 & -2 y & -\frac{1}{2} \\ -1 & -1 & z\end{array}\right)$ and $\psi_{2}(\mathbf{x})=\left(\begin{array}{c}x^{2}+\frac{1}{3} y+3 z \\ x+y^{2} \\ x^{2}+3 z\end{array}\right)$ with $P_{2}^{-T}=\left(\begin{array}{ccc}2 x & \frac{1}{3} & 3 \\ 1 & 2 y & 0 \\ 2 x & 0 & 3\end{array}\right)$

In Tables 5-8 we present some of the results obtained for $\psi_{1}$ and $\psi_{2}$ in the case $\frac{H}{h}=$ const. The results confirm the earlier observations.

Next, we increase $\frac{H}{h}$ while keeping the number of subdomains fixed. The results in Figures 6-9 match well with the theoretical estimates. It can be clearly seen that the square root of the maximum eigenvalue increases linearly with the logarithm of the subdomain size $\frac{H}{h}$. 
TABLE 8. $P^{-T}=\nabla \psi_{2}$, edge constraints without boundary and with vertex constraints.

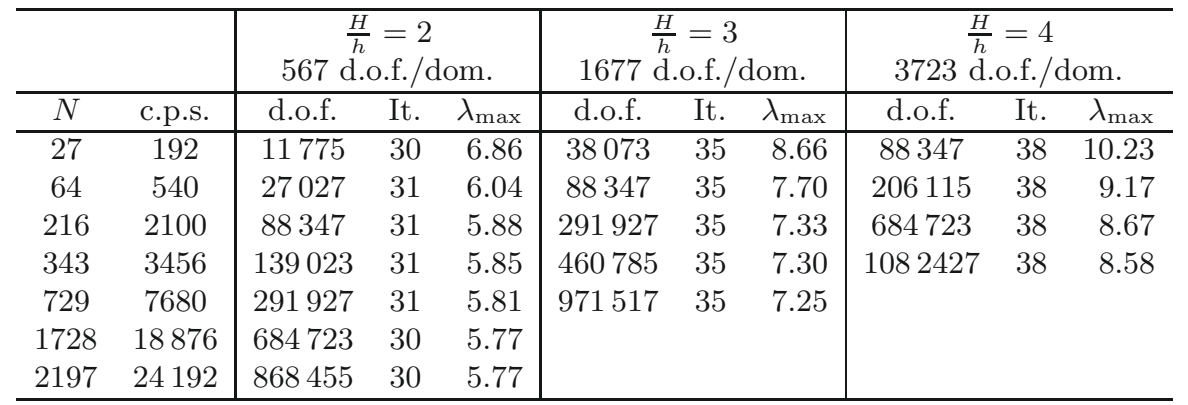

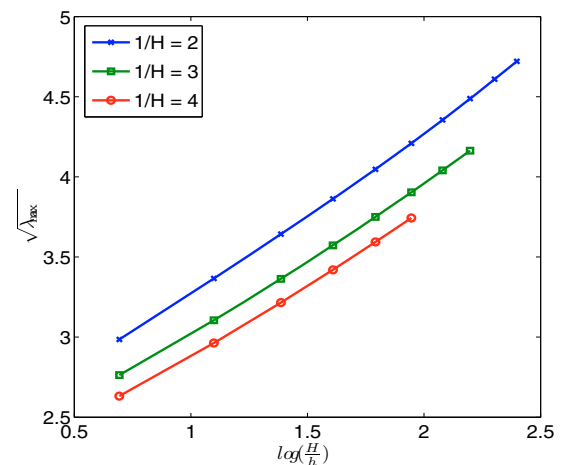

Figure $6 . P^{-T}=\nabla \psi_{1}$, edge constraints without edges on boundary and with vertex constraints.

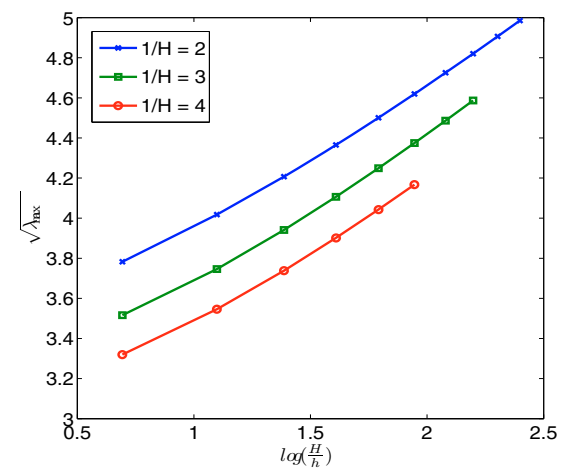

Figure 8. $P^{-T}=\nabla \psi_{2}$, edge constraints without edges on boundary.

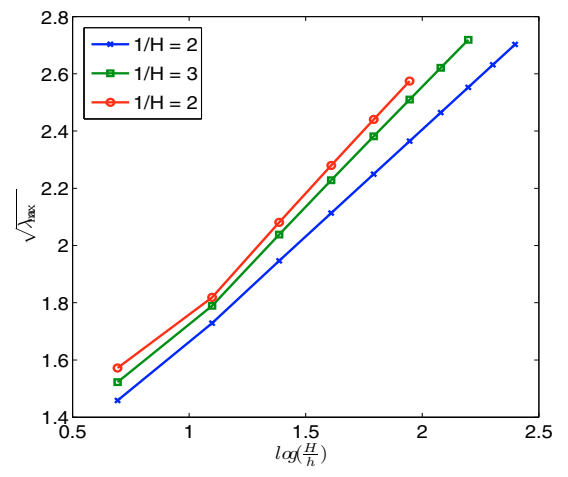

Figure 7. $P^{-T}=\nabla \psi_{1}$, edge constraints with edges on boundary and vertex constraints.

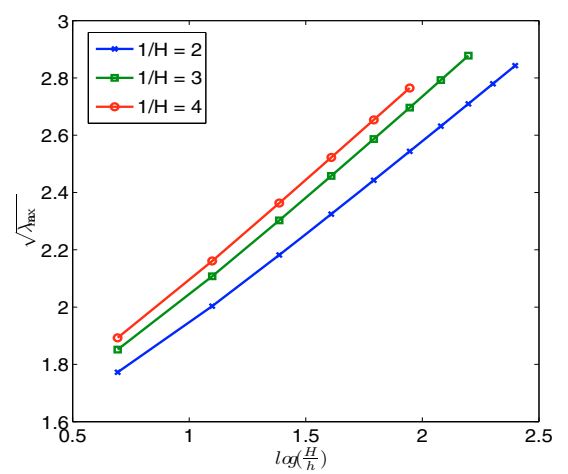

Figure 9. $P^{-T}=\nabla \psi_{2}$, edge constraints with edges on boundary. 


\subsection{Results for $\boldsymbol{P}^{-\boldsymbol{T}}=\nabla \psi$}

In this section we will present results for cases which do not completely match the assumptions made for our analysis in Section 7. The assumption that $P^{-T}$ is a gradient of a function $\psi: \mathbb{R}^{3} \rightarrow \mathbb{R}^{3}$ will be fulfilled. The function $\psi$ however does no longer consist of piecewise (at most) quadratic polynomials.

A special case, when only one entry of $\psi$ is not a polynomial with at most degree 2, will also be considered. Note that for the case discussed here, the infinitesimal rotations $\mathbf{r}_{4}(\mathbf{x}), \mathbf{r}_{5}(\mathbf{x}), \mathbf{r}_{6}(\mathbf{x})$, see (3.5), may not be representable exactly in the finite element space. As a consequence, the dimension of the kernel of the stiffness matrix may be different from six. The dimension is at least three since we can always represent exactly the translational basis vectors. But instead of the three zero eigenvalues associated with the three rotations we may have up to three additional positive eigenvalues. For example, in the case of $\psi_{4}$ the basis vector $\tilde{\mathbf{r}}_{4}$ is a composition of $\psi_{4}^{(1)}$ and $\psi_{4}^{(2)}$ which are quadratic polynomials. Hence, numerically we have a four dimensional kernel in this case.

The examples in this section can be divided into two parts. First, we consider the case when $\psi$ consists of polynomials of different degrees, i.e.,

$$
\begin{gathered}
\psi_{3}=\left(\begin{array}{c}
x^{3}+y \\
x^{3}+y+2 z \\
3 x+\frac{1}{9} z^{3}
\end{array}\right) \Rightarrow P_{3}^{-T}=\left(\begin{array}{ccc}
3 x^{2} & 1 & 0 \\
3 x^{2} & 1 & 2 \\
3 & 0 & \frac{1}{3} z^{2}
\end{array}\right) ; \psi_{4}=\left(\begin{array}{c}
x^{2}+\frac{1}{2} y+4 z \\
x^{2}+\frac{1}{2} y-6 z \\
-x+z^{3}
\end{array}\right) \Rightarrow P_{4}^{-T}=\left(\begin{array}{ccc}
2 x & \frac{1}{2} & 4 \\
2 x & \frac{1}{2} & -6 \\
-1 & 0 & 3 z^{2}
\end{array}\right) \\
\psi_{5}=\left(\begin{array}{c}
x^{3}-9 y+\frac{1}{3} z \\
4 x+2 y \\
x^{3}-y+\frac{1}{3} z
\end{array}\right) \Rightarrow P_{5}^{-T}=\left(\begin{array}{ccc}
3 x^{2}-9 & \frac{1}{3} \\
4 & 2 & 0 \\
3 x^{2}-1 & \frac{1}{3}
\end{array}\right),
\end{gathered}
$$

and then we consider a function $\psi$ which does not consist of polynomials

$$
\begin{aligned}
& \psi_{6}=\left(\begin{array}{c}
((1-h)+h x) \cos (2 \pi y) \cos (\alpha+z(\beta-\alpha)) \\
((1-h)+h x) \sin (2 \pi y) \cos (\alpha+z(\beta-\alpha)) \\
((1-h)+h x) \sin (\alpha+z(\beta-\alpha))
\end{array}\right)=:\left(\begin{array}{c}
A \cos (B) \cos (C) \\
A \sin (B) \cos (C) \\
A \sin (C)
\end{array}\right) \\
& \Rightarrow P_{6}^{-T}=\left(\begin{array}{ccc}
h \cos (B) \cos (C) & -2 \pi A \sin (B) \cos (C) & -(\beta-\alpha) A \cos (B) \sin (C) \\
h \sin (B) \cos (C) & 2 \pi A \cos (B) \cos (C) & -(\beta-\alpha) A \sin (B) \sin (C) \\
h \sin (C) & 0 & (\beta-\alpha) A \cos (C)
\end{array}\right) .
\end{aligned}
$$

Here, we considered two different sets of variables $h, \alpha$, and $\beta$. We will refer to the case with $h=\frac{1}{4}, \alpha=\frac{\pi}{8}$, and $\beta=\frac{\pi}{4}$ as $\psi_{6.1}$ and to the one with $h=\frac{1}{8}, \alpha=\frac{\pi}{16}$, and $\beta=\frac{3 \pi}{8}$ as $\psi_{6.2}$.

The results we obtained for $\psi_{3}, \psi_{4}$, and $\psi_{5}$ differ only slightly from the ones presented in Section 8.1, see Tables 9-12. In some cases the asymptotic range seems to be reached later and the condition number seems to vary more. Although these experiments are not covered by the theory, numerically, the bound for the condition number still seems to hold, and the number of iterations is clearly bounded. Again, a linear dependence of the square root of the maximum eigenvalue on $\log \left(\frac{H}{h}\right)$ can be observed numerically, see Figures 10-13.

The results obtained for $\psi_{6.1}$ and $\psi_{6.2}$, for $\frac{H}{h}$ kept fixed, match the theoretical expectations; cf. Tables 13 and 14 .

In the case when $\frac{H}{h}$ is increased and the number of subdomains is kept fixed, the bound for the condition number still seems to hold; $c f$. Figures 14 and 15 for $\psi_{6.1}$ and Figures 16 and 17 for $\psi_{6.2}$. The slope for the case $\frac{1}{H}=2$ in Figures 16 and 17 differs clearly from the cases $\frac{1}{H}=3$ and $\frac{1}{H}=4$. This suggests that the case $\frac{1}{H}=2$ is still away from the asymptotic range with respect to the number of subdomains. The results for $\frac{1}{H}=3$ and $\frac{1}{H}=4$, i.e., $N=27$ and $N=64$ subdomains are then very similar.

Summarizing the results in this section we can state that the numerical results differ only slightly from the results obtained in Section 8.1 although the theory does not apply. 
TABLE 9. $P^{-T}=\nabla \psi_{3}$, edge constraints without boundary edges and with vertex constraints.

\begin{tabular}{cc|ccc|ccc|crr}
\hline & & \multicolumn{3}{|c|}{$\frac{H}{h}=2$} & \multicolumn{3}{c|}{$\frac{H}{h}=3$} & \multicolumn{4}{c}{$\frac{H}{h}=4$} \\
& & \multicolumn{2}{|c|}{567 d.o.f./dom. } & \multicolumn{2}{c|}{1677} & d.o.f./dom. & \multicolumn{3}{c}{3723} & d.o.f./dom. \\
\hline$N$ & c.p.s. & d.o.f. & It. & $\lambda_{\max }$ & d.o.f. & It. & $\lambda_{\max }$ & d.o.f. & It. & $\lambda_{\max }$ \\
\hline 27 & 192 & 11775 & 30 & 7.16 & 38073 & 35 & 9.37 & 88347 & 38 & 11.26 \\
64 & 540 & 27027 & 31 & 6.56 & 88347 & 35 & 8.57 & 206115 & 39 & 10.28 \\
216 & 2100 & 88347 & 31 & 6.17 & 291927 & 35 & 7.99 & 684723 & 38 & 9.54 \\
343 & 3456 & 139023 & 31 & 6.06 & 460785 & 35 & 7.82 & 1082427 & 38 & 9.32 \\
729 & 7680 & 291927 & 31 & 5.90 & 971517 & 35 & 7.58 & & & \\
1728 & 18876 & 684723 & 31 & 5.76 & & & & & & \\
2197 & 24192 & 868455 & 30 & 5.73 & & & & & & \\
\hline
\end{tabular}

TABLE 10. $P^{-T}=\nabla \psi_{4}$, edge constraints with boundary edges.

\begin{tabular}{cc|ccc|ccc|cccc}
\hline & & \multicolumn{3}{|c|}{$\frac{H}{h}=2$} & \multicolumn{3}{c|}{$\frac{H}{h}=3$} & \multicolumn{4}{c}{$\frac{H}{h}=4$} \\
& & 567 & d.o.f./dom. & \multicolumn{2}{c|}{1677} & d.o.f./dom. & \multicolumn{3}{c}{3723} & d.o.f./dom. \\
\hline$N$ & c.p.s. & d.o.f. & It. & $\lambda_{\max }$ & d.o.f. & It. & $\lambda_{\max }$ & d.o.f. & It. & $\lambda_{\max }$ \\
\hline 27 & 288 & 11775 & 25 & 4.74 & 38073 & 29 & 5.72 & 88347 & 31 & 6.63 \\
64 & 684 & 27027 & 26 & 4.48 & 88347 & 29 & 5.50 & 206115 & 32 & 6.42 \\
216 & 2250 & 88347 & 25 & 4.04 & 291927 & 29 & 5.10 & 684723 & 32 & 6.04 \\
343 & 3528 & 139023 & 25 & 3.92 & 460785 & 29 & 5.03 & 1082427 & 32 & 5.99 \\
729 & 7344 & 291927 & 24 & 3.84 & 971517 & 28 & 5.02 & 2286795 & 32 & 5.99 \\
1728 & 17028 & 684723 & 24 & 3.82 & & & & & & \\
2197 & 21528 & 868455 & 24 & 3.83 & & & & & & \\
\hline
\end{tabular}

TABLE 11. $P^{-T}=\nabla \psi_{4}$, edge constraints with boundary edges and vertex constraints.

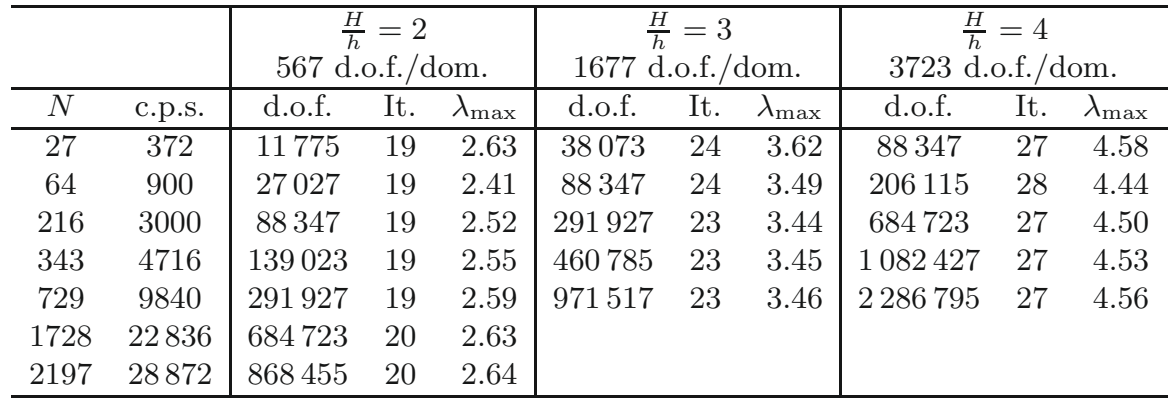

TABLE 12. $P^{-T}=\nabla \psi_{5}$, edge constraints with boundary edges.

\begin{tabular}{cc|ccc|ccc|cccc}
\hline & & \multicolumn{3}{|c|}{$\begin{array}{c}H \\
h\end{array}=2$} & \multicolumn{3}{c|}{$\frac{H}{h}=3$} & \multicolumn{4}{c}{$\frac{H}{h}=4$} \\
& & \multicolumn{2}{|c|}{567 d.o.f./dom. } & \multicolumn{2}{c|}{1677} & d.o.f./dom. & \multicolumn{3}{c}{3723} & d.o.f./dom. \\
\hline$N$ & c.p.s. & d.o.f. & It. & $\lambda_{\max }$ & d.o.f. & It. & $\lambda_{\max }$ & d.o.f. & It. & $\lambda_{\max }$ \\
\hline 27 & 288 & 11775 & 26 & 4.48 & 38073 & 29 & 5.79 & 88347 & 32 & 6.87 \\
64 & 684 & 27027 & 26 & 4.24 & 88347 & 30 & 5.50 & 206115 & 33 & 6.56 \\
216 & 2250 & 88347 & 25 & 3.95 & 291927 & 29 & 5.17 & 684723 & 33 & 6.18 \\
343 & 3528 & 139023 & 25 & 3.87 & 460785 & 29 & 5.12 & 1082427 & 33 & 6.12 \\
729 & 7344 & 291927 & 25 & 3.81 & 971517 & 29 & 5.07 & 2286795 & 32 & 6.07 \\
1728 & 17028 & 684723 & 25 & 3.85 & & & & & & \\
2197 & 21528 & 868455 & 24 & 3.81 & & & & & & \\
\hline
\end{tabular}




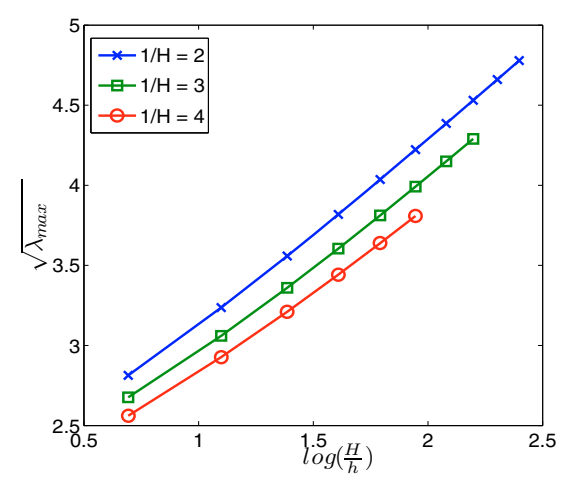

Figure 10. $P^{-T}=\nabla \psi_{3}$, edge constraints without edges on boundary and with vertex constraints.

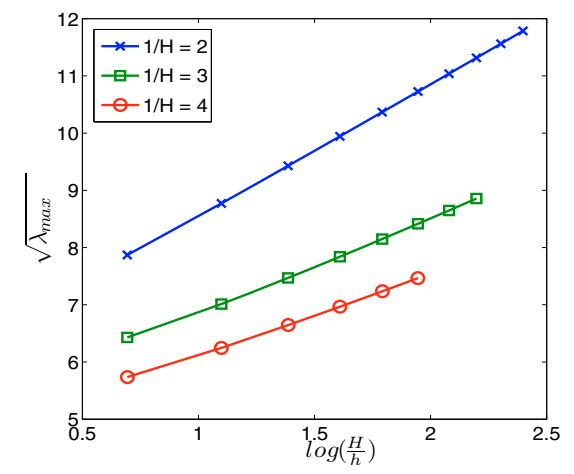

Figure 12. $P^{-T}=\nabla \psi_{4}$, edge constraints without edges on boundary.

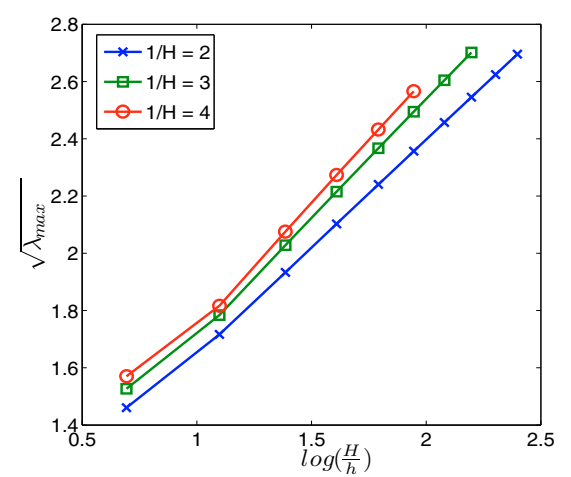

Figure 11. $P^{-T}=\nabla \psi_{3}$, edge constraints with edges on boundary and vertex constraints.

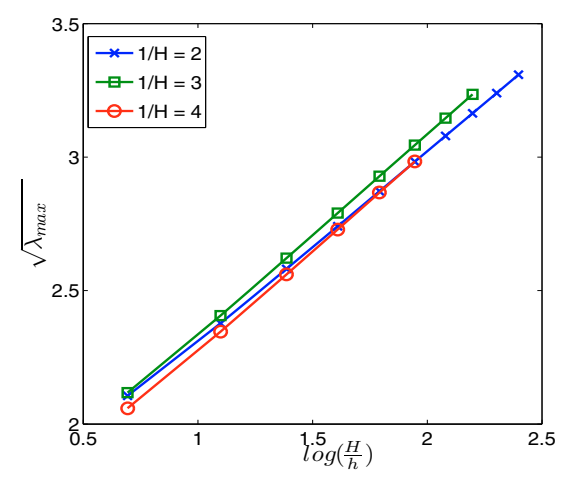

Figure 13. $P^{-T}=\nabla \psi_{5}$, edge constraints with edges on boundary.

TABLE 13. $P^{-T}=\nabla \psi_{6.1}$, edge constraints without boundary edges and with vertex constraints.

\begin{tabular}{|c|c|c|c|c|c|c|c|c|c|c|}
\hline \multirow[b]{2}{*}{$N$} & \multirow[b]{2}{*}{ c.p.s. } & \multicolumn{3}{|c|}{$\begin{array}{c}\frac{H}{h}=2 \\
567 \text { d.o.f./dom. } \\
\end{array}$} & \multicolumn{3}{|c|}{$\begin{array}{c}\frac{H}{h}=3 \\
1677 \text { d.o.f./dom. }\end{array}$} & \multicolumn{3}{|c|}{$\begin{array}{cl} & \frac{H}{h}=4 \\
3723 & \text { d.o.f./dom. }\end{array}$} \\
\hline & & d.o.f. & It. & $\lambda_{\max }$ & d.o.f. & It. & $\lambda_{\max }$ & d.o.f. & It. & $\lambda_{\max }$ \\
\hline 27 & 192 & 11775 & 42 & 16.18 & 38073 & 50 & 16.28 & 88347 & 57 & 19.38 \\
\hline 64 & 540 & 27027 & 43 & 14.54 & 88347 & 49 & 15.34 & 206115 & 55 & 18.23 \\
\hline 216 & 2100 & 88347 & 44 & 12.89 & 291927 & 47 & 14.44 & 684723 & 52 & 16.87 \\
\hline 343 & 3456 & 139023 & 43 & 12.24 & 460785 & 47 & 13.94 & 1082427 & 51 & 16.59 \\
\hline 729 & 7680 & 291927 & 42 & 11.03 & 971517 & 46 & 12.96 & & & \\
\hline 1728 & 18876 & 684723 & 40 & 9.51 & & & & & & \\
\hline 2197 & 24192 & 868455 & 39 & 9.32 & & & & & & \\
\hline
\end{tabular}




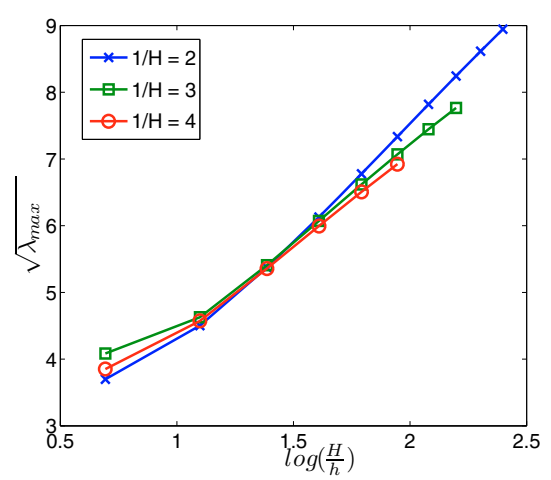

Figure 14. $P^{-T}=\nabla \psi_{6.1}$, edge constraints with edges on boundary.

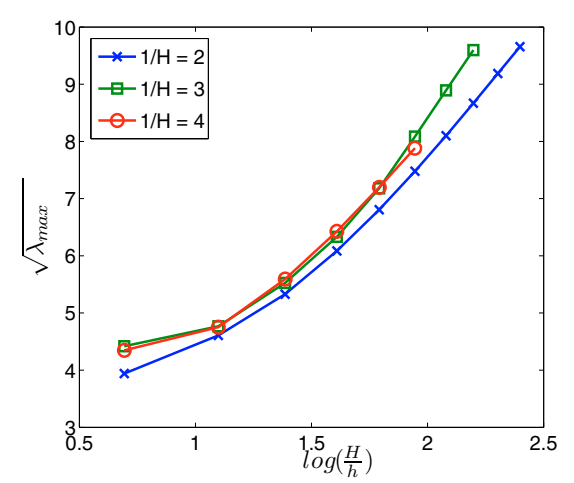

Figure 16. $P^{-T}=\nabla \psi_{6.2}$, edge constraints with edges on boundary.

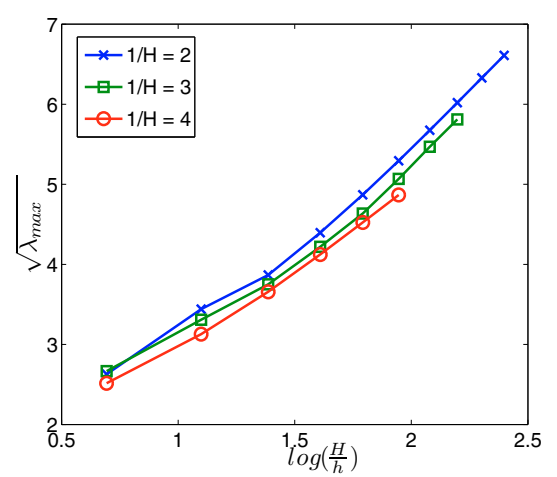

Figure 15. $P^{-T}=\nabla \psi_{6.1}$, edge constraints with edges on boundary and vertex constraints.

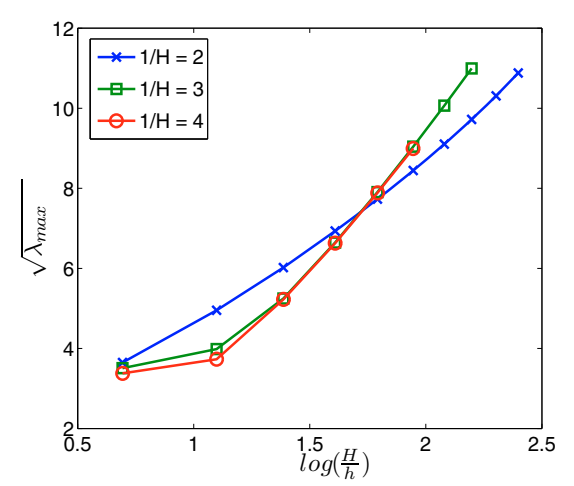

Figure 17. $P^{-T}=\nabla \psi_{6.2}$, edge constraints without edges on boundary and with vertex constraints.

TABLE 14. $P^{-T}=\nabla \psi_{6.2}$, edge constraints without boundary edges and with vertex constraints.

\begin{tabular}{|c|c|c|c|c|c|c|c|c|c|c|}
\hline \multirow[b]{2}{*}{$N$} & \multirow[b]{2}{*}{ c.p.s. } & \multicolumn{3}{|c|}{$\begin{array}{c}\frac{H}{h}=2 \\
567 \text { d.o.f./dom. } \\
\end{array}$} & \multicolumn{3}{|c|}{$\begin{array}{cl} & \frac{H}{h}=3 \\
1677 & \text { d.o.f./dom. }\end{array}$} & \multicolumn{3}{|c|}{$\begin{array}{c}\frac{H}{h}=4 \\
3723 \text { d.o.f./dom. }\end{array}$} \\
\hline & & d.o.f. & It. & $\lambda_{\max }$ & d.o.f. & It. & $\lambda_{\max }$ & d.o.f. & It. & $\lambda_{\max }$ \\
\hline 27 & 192 & 11775 & 42 & 12.31 & 38073 & 53 & 15.87 & 88347 & 65 & 27.54 \\
\hline 64 & 540 & 27027 & 42 & 11.41 & 88347 & 51 & 13.92 & 206115 & 66 & 27.28 \\
\hline 216 & 2100 & 88347 & 42 & 9.91 & 291927 & 48 & 12.35 & 684723 & 61 & 21.07 \\
\hline 343 & 3456 & 139023 & 41 & 9.40 & 460785 & 47 & 11.95 & 1082427 & 58 & 18.36 \\
\hline 729 & 7680 & 291927 & 40 & 8.74 & 971517 & 46 & 11.56 & & & \\
\hline 1728 & 18876 & 684723 & 39 & 8.35 & & & & & & \\
\hline 2197 & 24192 & 868455 & 38 & 8.37 & & & & & & \\
\hline
\end{tabular}




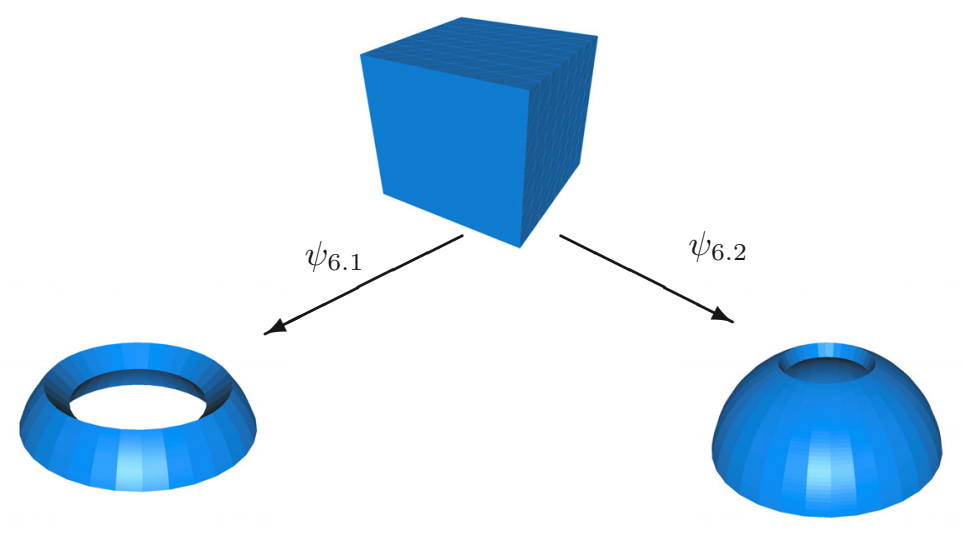

Figure 18. Deformations induced by $P_{6.1}$ and $P_{6.2}$.
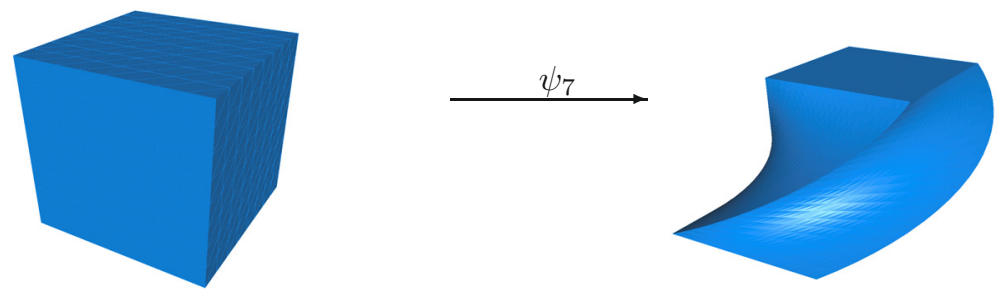

Figure 19. Deformation induced by $P_{7}$

\subsection{More general cases}

Here, we will discuss results obtained for the case that $P$ itself is a gradient, i.e., $P=\nabla \psi$. We know that the solution of the minimizing problem in $\varphi$ is then given by $\varphi=\psi$. The examples in this section do not match the assumptions for our analysis. The functions $\psi_{6.1}$ and $\psi_{6.2}$ introduced in Section 8.2 transform the cube into a spherical dome with different thickness and angles if $P=\nabla \psi_{6.1}$ or $P=\nabla \psi_{6.2}$; see Figure 18. Here, in addition to the aforementioned Dirichlet boundary conditions we introduce further Dirichlet boundary conditions for the $y$-direction on $\left\{\mathbf{x} \in \mathbb{R}^{3}: y \in\{0,1\}\right\}$ to prevent small gaps or element overlaps coming from inaccuracies of the numerical solution. Another example for $P=\nabla \psi$ is given by $\psi_{7}$

$$
\psi_{7}(\mathbf{x})=\left(\begin{array}{c}
x \cos \left(\frac{\pi}{2} z\right)-y \sin \left(\frac{\pi}{2} z\right) \\
x \sin \left(\frac{\pi}{2} z\right)+y \cos \left(\frac{\pi}{2} z\right) \\
z
\end{array}\right)
$$

which describes a linear increasing twist of the unit cube; see Figure 19.

The results for $P=\nabla \psi_{7}$ in the case of a constant subdomain size match the expectations from the theory in Section 7 even though the assumptions do not match. For growing $\frac{1}{H}$ and fixed $\frac{H}{h}$ the condition and iteration numbers are clearly bounded by a constant; $c f$. Tables $15-17$.

For $\psi_{6.1}$ and $\psi_{6.2}$ we obtain similar results for fixed $\frac{H}{h}$; see Tables 18 and 19, where the results are given for sets of primal variables which combine edge averages and vertex constraints.

In Figure 20 the behavior for an increasing $\frac{H}{h}$ is shown for $\psi_{6.1}$ for the set of primal variables consisting of edge averages including boundary edges and combined with vertex constraints. In Figure 21 results are shown for $\psi_{6.2}$. The results are very similar to the ones obtained in the previous section. See Figures 22 and 23 
TABLE 15. $P=\nabla \psi_{7}$, edge constraints with boundary edges.

\begin{tabular}{|c|c|c|c|c|c|c|c|c|c|c|}
\hline \multirow[b]{2}{*}{$N$} & \multirow[b]{2}{*}{ c.p.s. } & \multicolumn{3}{|c|}{$\begin{array}{c}\frac{H}{h}=2 \\
567 \text { d.o.f./dom. }\end{array}$} & \multicolumn{3}{|c|}{$\begin{array}{ll} & \frac{H}{h}=3 \\
1677 & \text { d.o.f./dom. }\end{array}$} & \multicolumn{3}{|c|}{$\begin{array}{c}\frac{H}{h}=4 \\
3723 \text { d.o.f./dom. }\end{array}$} \\
\hline & & d.o.f. & It. & $\lambda_{\max }$ & d.o.f. & It. & $\lambda_{\max }$ & d.o.f. & It. & $\lambda_{\max }$ \\
\hline 27 & 288 & 11775 & 22 & 3.45 & 38073 & 25 & 4.38 & 88347 & 28 & 5.21 \\
\hline 64 & & 27027 & 23 & 3. & 88347 & 26 & 4. & 206115 & 29 & 5.49 \\
\hline 216 & 2250 & 88347 & 23 & 3.72 & 291927 & 27 & 4.82 & 684723 & 30 & 5.75 \\
\hline 343 & 3528 & 139023 & 23 & 3.75 & 460785 & 27 & 4.89 & 1082427 & 30 & 5.85 \\
\hline 729 & 7344 & 291927 & 23 & 3.78 & 971517 & 27 & 4.95 & & & \\
\hline 1728 & 17028 & 684723 & 23 & 3.82 & & & & & & \\
\hline 2197 & 21528 & 868455 & 23 & 3.78 & & & & & & \\
\hline
\end{tabular}

TABLE 16. $P=\nabla \psi_{7}$, edge constraints without boundary and vertex constraints.

\begin{tabular}{cc|ccc|ccc|crr}
\hline & & \multicolumn{3}{|c|}{$\frac{H}{h}=2$} & \multicolumn{3}{c|}{$\frac{H}{h}=3$} & \multicolumn{3}{c}{$\frac{H}{h}=4$} \\
& & \multicolumn{2}{|c|}{567 d.o.f./dom. } & \multicolumn{2}{c|}{1677} & d.o.f./dom. & \multicolumn{3}{c}{3723} & d.o.f./dom. \\
\hline$N$ & c.p.s. & d.o.f. & It. & $\lambda_{\max }$ & d.o.f. & It. & $\lambda_{\max }$ & d.o.f. & It. & $\lambda_{\max }$ \\
\hline 27 & 192 & 11775 & 30 & 7.80 & 38073 & 34 & 9.81 & 88347 & 37 & 11.37 \\
64 & 540 & 27027 & 31 & 6.82 & 88347 & 34 & 8.54 & 206115 & 37 & 9.92 \\
216 & 2100 & 88347 & 31 & 6.22 & 291927 & 34 & 7.91 & 684723 & 37 & 9.21 \\
343 & 3456 & 139023 & 31 & 6.20 & 460785 & 35 & 7.75 & 1082427 & 37 & 9.03 \\
729 & 7680 & 291927 & 31 & 6.03 & 971517 & 34 & 7.53 & & & \\
1728 & 18876 & 684723 & 31 & 5.91 & & & & & & \\
2197 & 24192 & 868455 & 31 & 5.90 & & & & & & \\
\hline
\end{tabular}

TABLE 17. $P=\nabla \psi_{7}$, edge constraints with boundary and vertex constraints.

\begin{tabular}{cc|ccc|ccc|cccc}
\hline & & \multicolumn{3}{|c|}{$\begin{array}{c}H \\
h\end{array}=2$} & \multicolumn{3}{c|}{$\frac{H}{h}=3$} & \multicolumn{4}{c}{$\frac{H}{h}=4$} \\
& & \multicolumn{2}{|c|}{567 d.o.f./dom. } & \multicolumn{2}{c|}{1677} & d.o.f./dom. & \multicolumn{3}{c}{3723} & d.o.f./dom. \\
\hline$N$ & c.p.s. & d.o.f. & It. & $\lambda_{\max }$ & d.o.f. & It. & $\lambda_{\max }$ & d.o.f. & It. & $\lambda_{\max }$ \\
\hline 27 & 372 & 11775 & 17 & 2.34 & 38073 & 21 & 3.18 & 88347 & 24 & 4.13 \\
64 & 900 & 27027 & 18 & 2.49 & 88347 & 22 & 3.29 & 206115 & 25 & 4.32 \\
216 & 3000 & 88347 & 19 & 2.60 & 291927 & 22 & 3.37 & 684723 & 26 & 4.43 \\
343 & 4716 & 139023 & 19 & 2.63 & 460785 & 22 & 3.39 & 1082427 & 26 & 4.48 \\
729 & 9840 & 291927 & 19 & 2.66 & 971517 & 22 & 3.39 & & & \\
1728 & 22836 & 684723 & 19 & 2.69 & & & & & & \\
2197 & 28872 & 868455 & 19 & 2.69 & & & & & & \\
\hline
\end{tabular}

TABLE 18. $P=\nabla \psi_{6.1}$, edge constraints with boundary edges and vertex constraints.

\begin{tabular}{cc|ccc|ccc|ccc}
\hline & & \multicolumn{3}{|c|}{$\frac{H}{h}=2$} & \multicolumn{3}{c|}{$\frac{H}{h}=3$} & \multicolumn{3}{c}{$\frac{H}{h}=4$} \\
& & \multicolumn{2}{|c|}{567 d.o.f./dom. } & \multicolumn{2}{c|}{1677} & d.o.f./dom. & \multicolumn{3}{c}{3723 d.o.f./dom. } \\
\hline$N$ & c.p.s. & d.o.f. & It. & $\lambda_{\max }$ & d.o.f. & It. & $\lambda_{\max }$ & d.o.f. & It. & $\lambda_{\max }$ \\
\hline 27 & 292 & 11775 & 30 & 5.67 & 38073 & 40 & 11.87 & 88347 & 47 & 19.00 \\
64 & 738 & 27027 & 30 & 6.10 & 88347 & 43 & 12.74 & 206115 & 54 & 20.20 \\
216 & 2590 & 88347 & 31 & 5.69 & 291927 & 44 & 11.57 & 684723 & 55 & 17.89 \\
343 & 4140 & 139023 & 31 & 5.54 & 460785 & 44 & 10.96 & 1082427 & 54 & 16.82 \\
729 & 8848 & 291927 & 30 & 5.21 & 971517 & 42 & 10.02 & & & \\
1728 & 21010 & 684723 & 28 & 4.80 & & & & & & \\
2197 & 26712 & 868455 & 28 & 4.67 & & & & & & \\
\hline
\end{tabular}


TABLE 19. $P=\nabla \psi_{6.2}$, edge constraints without boundary edges and with vertex constraints.

\begin{tabular}{cc|ccc|ccc|ccc}
\hline & & \multicolumn{3}{|c|}{$\frac{H}{h}=2$} & \multicolumn{3}{c|}{$\frac{H}{h}=3$} & \multicolumn{3}{c}{$\frac{H}{h}=4$} \\
& & \multicolumn{2}{|c|}{567 d.o.f./dom. } & \multicolumn{2}{c|}{1677 d.o.f./dom. } & \multicolumn{3}{c}{3723 d.o.f./dom. } \\
\hline$N$ & c.p.s. & d.o.f. & It. & $\lambda_{\max }$ & d.o.f. & It. & $\lambda_{\max }$ & d.o.f. & It. & $\lambda_{\max }$ \\
\hline 27 & 184 & 11775 & 33 & 7.82 & 38073 & 45 & 15.24 & 88347 & 55 & 24.31 \\
64 & 522 & 27027 & 33 & 7.74 & 88347 & 48 & 15.29 & 206115 & 61 & 24.30 \\
216 & 2050 & 88347 & 34 & 7.03 & 291927 & 48 & 13.38 & 684723 & 60 & 21.41 \\
343 & 3384 & 139023 & 34 & 6.71 & 460785 & 47 & 12.94 & 1082427 & 59 & 20.65 \\
729 & 7552 & 291927 & 32 & 6.39 & 971517 & 47 & 12.46 & & & \\
1728 & 18634 & 684723 & 32 & 6.15 & & & & & & \\
2197 & 23904 & 868455 & 31 & 6.10 & & & & & & \\
\hline
\end{tabular}

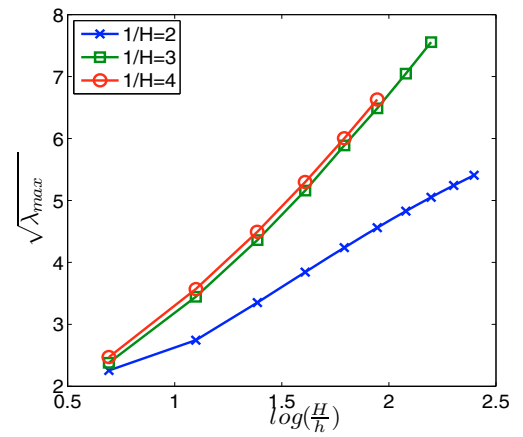

Figure 20. $P=\nabla \psi_{6.1}$, edge constraints with edges on boundary and vertex constraints.

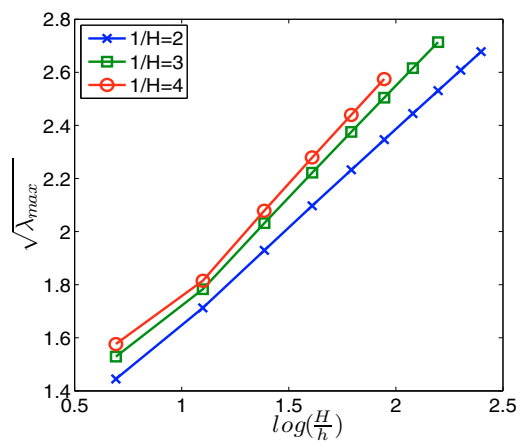

Figure 22. $P=\nabla \psi_{7}$, edge constraints with edges on boundary and vertex constraints.

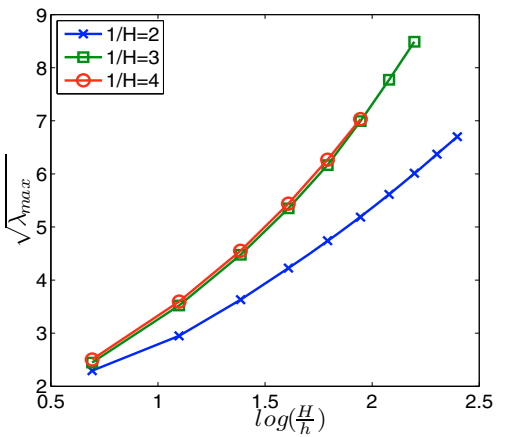

Figure 21. $P=\nabla \psi_{6.2}$, edge constraints without edges on boundary and with vertex constraints.

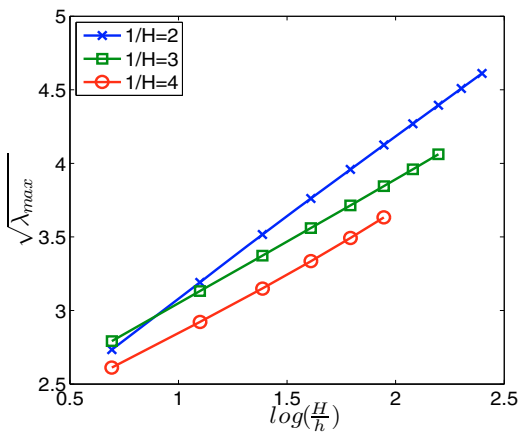

Figure 23. $P=\nabla \psi_{7}$, edge constraints without edges on boundary and with vertex constraints.

for results for $\psi_{7}$ which are numerically in accordance with the theoretical findings although the theory does not apply. 


\section{A. Appendix: Some auxiliary lemmas}

Here, we give some of the technical lemmas needed for our convergence analysis in Section 7 . Some of them are provided with proofs. The proofs for Lemma A.3 and A.4 can be found in [31], Section 5.

Lemma A.1 is related to earlier lemmas for scalar functions and standard linear elasticity; see Dryja et al. [11], Lemma 4.4, Klawonn and Widlund [26], Lemma 7.1, and also the book of Toselli and Widlund [55], Lemma 4.25. Since we are using piecewise quadratic finite element functions and $P$-elasticity these lemmas are not applicable here. Thus, we present a new version for the rigid body modes of linear $P$-elasticity and piecewise quadratic finite element functions.

Lemma A.1. Let $\mathcal{F}^{i j}$ be the face common to $\Omega_{i}$ and $\Omega_{j}$ and let $\theta_{\mathcal{F}^{i j}}$ be the piecewise linear finite element function on the triangulation $\tau_{h / 2}$ introduced in Section 7 that is equal to 1 at the nodal points on the face $\mathcal{F}^{i j}=\mathcal{F}_{h / 2}^{i j}$ and vanishes on $\left(\partial \Omega_{i, h / 2} \cup \partial \Omega_{j, h / 2}\right) \backslash \mathcal{F}_{h / 2}^{i j}$. In the interior of $\Omega_{i}$ and $\Omega_{j}, \theta_{\mathcal{F}^{i j}}$ is assumed to be the discrete harmonic extension of the given values on the boundary. Furthermore, let $\mathbf{r} \in\left\{\mathbf{r}_{1}, \ldots, \mathbf{r}_{6}\right\}$ be a rigid body mode, cf. (3.5), with $\psi$ being at most piecewise quadratic. Then

$$
\left|I^{h}\left(\theta_{\mathcal{F}^{i j}} \mathbf{r}\right)\right|_{H^{1 / 2}\left(\partial \Omega_{i}\right)}^{2} \leq C\left(1+\log \left(\frac{H_{i}}{h_{i}}\right)\right) H_{i}
$$

Proof. From (6.9) and (6.10) follows

$$
\left|I^{h}\left(\theta_{\mathcal{F}^{i j}} \mathbf{r}\right)\right|_{H^{1 / 2}\left(\partial \Omega_{i}\right)}^{2} \leq\left|I^{h}\left(\theta_{\mathcal{F}^{i j}} \mathbf{r}\right)\right|_{H^{1}\left(\Omega_{i}\right)}^{2} .
$$

Since $\theta_{\mathcal{F}^{i j}} \mathbf{r}$ is at most piecewise cubic, we can follow the arguments given in [55], Lemma 3.9, and obtain for $\mathbf{r}^{T}=\left(r^{(1)}, r^{(2)}, r^{(3)}\right)^{T}$ that

$$
\left|I^{h}\left(\theta_{\mathcal{F}^{i j}} \mathbf{r}\right)\right|_{H^{1}\left(\Omega_{i}\right)}^{2} \leq C\left|\theta_{\mathcal{F}^{i j}} \mathbf{r}\right|_{H^{1}\left(\Omega_{i}\right)}^{2}=\sum_{k=1}^{3}\left|\theta_{\mathcal{F}^{i j}} r^{(k)}\right|_{H^{1}\left(\Omega_{i}\right)}^{2},
$$

cf. [55], Lemma 4.31, by summing over the elements $T$ of the triangulation. Thus, for $k=1,2,3$, we have to estimate

$$
\left|\theta_{\mathcal{F}^{i j}} r^{(k)}\right|_{H^{1}\left(\Omega_{i}\right)}^{2}=\int_{\Omega_{i}}\left|\left(\nabla \theta_{\mathcal{F}^{i j}}\right) r^{(k)}+\theta_{\mathcal{F}^{i j}}\left(\nabla r^{(k)}\right)\right|^{2} \mathrm{~d} x \leq 2\left(\int_{\Omega_{i}}\left|\nabla \theta_{\mathcal{F}^{i j}}\right|^{2}\left|r^{(k)}\right|^{2} \mathrm{~d} x+\int_{\Omega_{i}}\left|\theta_{\mathcal{F}^{i j}}\right|^{2}\left|\nabla r^{(k)}\right|^{2} \mathrm{~d} x\right)
$$

For the first term in (A.1) we can use that the shifted version of the rigid body modes $\mathbf{r}, c f$. (3.6), are constructed such that $\left\|r^{(k)}\right\|_{L^{\infty}\left(\Omega_{i}\right)} \leq C$ with a constant $C$ independent of $H_{i}$ and $h_{i}$. Thus, we obtain

$$
\int_{\Omega_{i}}\left|\nabla \theta_{\mathcal{F}^{i j}}\right|^{2}\left|r^{(k)}\right|^{2} \mathrm{~d} x \leq C\left|\theta_{\mathcal{F}^{i j}}\right|_{H^{1}\left(\Omega_{i}\right)}^{2} \leq \tilde{C}\left(1+\log \left(\frac{H_{i}}{\frac{h_{i}}{2}}\right)\right) H_{i} \leq(1+\log (2)) \tilde{C}\left(1+\log \left(\frac{H_{i}}{h_{i}}\right)\right) H_{i}
$$

where the penultimate inequality can be found in [55], Lemma 4.25.

The second term in (A.1) can be bounded by first representing the integral over $\Omega_{i}$ as the sum of the integrals over all elements $T \in \tau_{h}$ with $T \cap \Omega_{i} \neq \emptyset$. Then, we obtain

$$
\int_{\Omega_{i}}\left|\theta_{\mathcal{F}^{i j}}\right|^{2}\left|\nabla r^{(k)}\right|^{2} \mathrm{~d} x=\sum_{T \subset \Omega_{i}} \int_{T}\left|\theta_{\mathcal{F}^{i j}}\right|^{2}\left|\nabla r^{(k)}\right|^{2} \mathrm{~d} x \leq \sum_{T \subset \Omega_{i}} \int_{T}\left|\nabla r^{(k)}\right|^{2} \mathrm{~d} x,
$$

where we used that $\left|\theta_{\mathcal{F}^{i j}}(x)\right| \leq 1$. Now we consider that $\mathbf{r}$ is a rigid body mode of $P$-elasticity, i.e.,

$$
\mathbf{r}(\mathbf{x})=\mathbf{r}_{i}(\mathbf{x})=\tilde{\mathbf{r}}_{i}(\psi(\mathbf{x})),
$$


with $\tilde{\mathbf{r}}_{i}, i=1, \ldots, 6$, being the rigid body modes of standard linear elasticity. Thus, we have

$$
\nabla_{\mathbf{x}} \mathbf{r}(\mathbf{x})=\left(\nabla_{\mathbf{y}} \tilde{\mathbf{r}}_{i}(\mathbf{y})\right)\left(\nabla_{\mathbf{x}} \psi(\mathbf{x})\right)=\left(\nabla_{\mathbf{y}} \tilde{\mathbf{r}}_{i}(\mathbf{y})\right) P^{-T} \text { with } \mathbf{y}:=\psi(\mathbf{x}) .
$$

Since the $\tilde{\mathbf{r}}_{i}, i=1, \ldots, 6$, have elements which are at most linear functions their derivatives are either constant or zero. Hence, we obtain

$$
\int_{T}\left|\nabla r^{(k)}\right|^{2} \mathrm{~d} x \leq \hat{C} c_{P}^{2} \int_{T} 1 \mathrm{~d} \mathbf{x}=\hat{C} c_{P}^{2}|T|,
$$

with $c_{P}$ as defined in (6.6) and $|T|$ being the measure of the element $T$. Since $\log \left(\frac{H_{i}}{h_{i}}\right)$ is positive, $|T| \leq h_{i}^{3}$, and $h_{i}<1$ we have $|T| \leq h_{i}^{3} \leq h_{i} \leq H_{i} \leq H_{i}\left(1+\log \left(\frac{H_{i}}{h_{i}}\right)\right)$.

Hence, we have

$$
\left|I^{h}\left(\theta_{\mathcal{F}^{i j}} \mathbf{r}\right)\right|_{H^{1 / 2}\left(\partial \Omega_{i}\right)}^{2} \leq \max \left\{(1+\log (2)) \tilde{C}, \hat{C} c_{P}^{2}\right\} H_{i}\left(1+\log \left(\frac{H_{i}}{h_{i}}\right)\right) .
$$

We also need two additional results to estimate the contribution to our bounds from the edges of $\Omega_{i}$. For a version in the context of piecewise linear finite elements, see [11], Lemma 4.7, and [55], Lemma 4.19. Here, we provide a version for piecewise quadratic finite element functions using a partition of unity $\theta_{\mathcal{E}^{i k}}$ which is piecewise linear on a mesh with element size $h / 2$; see also Section 7 .

Lemma A.2. Let $\theta_{\mathcal{E}^{i k}}$ be the piecewise linear function that is equal to 1 at the nodal points on the edge $\mathcal{E}_{h / 2}^{i k}$ and vanishes on $\left(\partial \Omega_{i, h / 2} \cup \partial \Omega_{j, h / 2}\right) \backslash \mathcal{E}_{h / 2}^{i k}$. Then, for all $u \in W^{(i)}$,

$$
\left|I^{h}\left(\theta_{\mathcal{E}^{i k}} \mathbf{u}\right)\right|_{H^{1 / 2}\left(\partial \Omega_{i}\right)}^{2} \leq C\|\mathbf{u}\|_{L_{2}\left(\mathcal{E}^{i k}\right)}^{2} .
$$

Proof. As before we prove the estimate for the $H^{1}\left(\Omega_{i}\right)$-seminorm and obtain our result for the $H^{1 / 2}\left(\partial \Omega_{i}\right)$ seminorm using (6.9) and (6.10). Since $I^{h}\left(\theta_{\mathcal{E}^{i k}} \mathbf{u}\right)$ is a finite element function in $\mathbf{W}^{\mathbf{h}}$, we have

$$
I^{h}\left(\theta_{\mathcal{E}^{i k}} \mathbf{u}\right)=\sum_{j}\left(\theta_{\mathcal{E}^{i k}} \mathbf{u}\right)\left(P_{j}\right) \phi_{j}
$$

where $P_{j}$ are the nodes of the triangulation and $\phi_{j}=\left(\phi_{j, q}\right), q=1,2,3$, where $\left(\phi_{j, q}\right)$ is the piecewise quadratic nodal basis function associated with $P_{j}$. Using Proposition 3.4.1 in [48] we can bound $\left|\phi_{j, q}\right|_{H^{1}(T)}^{2}$ as follows

$$
c h_{T} \leq\left|\phi_{j, q}\right|_{H^{1}(T)}^{2} \leq C h_{T}
$$

where the constants $c, C$ depend on the $H^{1}\left(T_{\text {ref }}\right)$-seminorms of the reference basis functions.

Let $T \in \tau_{h}, T \subset \bar{\Omega}_{i}$ be an element of the triangulation such that $\partial T \cap \mathcal{E}^{i k} \neq \emptyset$ is a straight line from a point $a \in \mathbb{R}^{3}$ to a point $b \in \mathbb{R}^{3}$. Then, for $\mathbf{u}^{T}=\left(u_{1}, u_{2}, u_{3}\right)^{T}$ and $q=1,2,3$, we have

$$
\begin{aligned}
\left|I^{h}\left(\theta_{\mathcal{E}^{i k}} u_{q}\right)\right|_{H^{1}(T)}^{2} \leq C \sum_{j=1}^{10}\left|\left(\theta_{\mathcal{E}^{i k}} u_{q}\right)\left(P_{j}\right)\right|^{2}\left|\phi_{j, q}\right|_{H^{1}(T)}^{2} & \leq C h_{T}\left(u_{q}^{2}(a)+u_{q}^{2}(b)+u_{q}^{2}\left(\frac{a+b}{2}\right)\right) \\
& \leq C \int_{\mathcal{E}^{i k}}\left|u_{q}(x)\right|^{2} \mathrm{~d} x=c\left\|u_{q}\right\|_{L_{2}\left(\mathcal{E}^{i k}\right)}^{2} .
\end{aligned}
$$

We obtain our result by summing over the elements belonging to the subdomain $\Omega_{i}$ and using (6.9) and (6.10). 
We also need a Sobolev-type inequality for piecewise quadratic finite element functions. The proof for piecewise quadratic functions can essentially be carried out as in the version for piecewise linear finite element functions; $c f$. Toselli and Widlund [55], Lemma 4.16, see also [31], Section 5, Lemma 2, for a detailed proof.

Lemma A.3. Let $\mathcal{E}^{i k}$ be any edge of $\Omega_{i}$ that forms a part of the boundary of a face $\mathcal{F}^{i j} \subset \partial \Omega_{i}$. Then for all $\mathbf{u} \in \mathbf{W}^{(\mathbf{i})}$,

$$
\|\mathbf{u}\|_{L_{2}(\mathcal{E} i k)}^{2} \leq C\left(1+\log \left(\frac{H_{i}}{h_{i}}\right)\right)\left(|\mathbf{u}|_{H^{1 / 2}\left(\partial \Omega_{i}\right)}^{2}+\frac{1}{H_{i}}\|\mathbf{u}\|_{L_{2}\left(\partial \Omega_{i}\right)}^{2}\right) .
$$

The next lemma can be found in the monograph by Toselli and Widlund [55], Lemma 4.28, for the case of piecewise linear finite element functions. The proof for piecewise quadratic finite element functions is essentially the same, see [31], Section 5, Lemma 4, for a detailed proof.

Lemma A.4. Let $\mathcal{V}^{\text {il }}$ be a vertex of a subdomain $\Omega_{i}$ and let $\mathbf{u} \in \mathbf{W}^{(\mathbf{i})}$. Then

$$
\left|\mathbf{u}\left(\mathcal{V}^{i l}\right) \theta_{\mathcal{V}^{i l}}\right|_{H^{1 / 2}\left(\partial \Omega_{i}\right)}^{2} \leq C\left(|\mathbf{u}|_{H^{1 / 2}\left(\partial \Omega_{i}\right)}^{2}+\frac{1}{H_{i}}\|\mathbf{u}\|_{L_{2}\left(\partial \Omega_{i}\right)}^{2}\right) .
$$

The following result can be found in Dryja et al. [11], Lemma 4.5, Dryja [10], Lemma 3, and Toselli and Widlund [55], Lemma 4.24, but only for piecewise linear functions. Here, we present a version for piecewise quadratic finite element functions. For this case, it can be proven by combining the arguments given in the proof of [55], Lemma 4.24, with the same element by element techniques as applied for the previous lemmas of this section.

Lemma A.5. Let $\theta_{\mathcal{F}^{i j}}$ be the function introduced in Lemma A.1. For all $\mathbf{u} \in \mathbf{W}^{(\mathbf{i})}$,

$$
\left|I^{h}\left(\theta_{\mathcal{F}^{i j}} \mathbf{u}\right)\right|_{H^{1 / 2}\left(\partial \Omega_{i}\right)}^{2} \leq C\left(1+\log \left(\frac{H_{i}}{h_{i}}\right)\right)^{2}\left(|\mathbf{u}|_{H^{1 / 2}\left(\partial \Omega_{i}\right)}^{2}+\frac{1}{H_{i}}\|\mathbf{u}\|_{L_{2}\left(\partial \Omega_{i}\right)}^{2}\right) .
$$

\section{REFERENCES}

[1] S. Balay, W.D. Gropp, L.C. McInnes and B.F. Smith, Efficient management of parallelism in object oriented numerical software libraries, in Modern Software Tools in Scientific Computing, E. Arge, A.M. Bruaset and H.P. Langtangen Eds., Birkhäuser Press (1997) 163-202.

[2] S. Balay, K. Buschelman, W.D. Gropp, D. Kaushik, M. Knepley, L.C. McInnes, B.F. Smith and H. Zhang, PETSc users manual. Technical Report ANL-95/11 - Revision 2.2.3, Argonne National Laboratory (2007).

[3] S. Balay, K. Buschelman, W.D. Gropp, D. Kaushik, M.G. Knepley, L.C. McInnes, B.F. Smith and H. Zhang, PETSc Web page, http://www.mcs.anl.gov/petsc (2009).

[4] J.M. Ball, Constitutive inequalities and existence theorems in nonlinear elastostatics, in Herriot Watt Symposion: Nonlinear Analysis and Mechanics 1, R.J. Knops Ed., Pitman, London (1977) 187-238.

[5] J.M. Ball, Convexity conditions and existence theorems in nonlinear elasticity. Arch. Rat. Mech. Anal. 63 (1977) $337-403$.

[6] J.M. Ball, Some open problems in elasticity, in Geometry, mechanics, and dynamics, P. Newton, P. Holmes and A. Weinstein Eds., Springer, New York (2002) 3-59.

[7] D. Balzani, P. Neff, J. Schröder and G.A. Holzapfel, A polyconvex framework for soft biological tissues. Adjustment to experimental data. Int. J. Solids Struct. 43 (2006) 6052-6070.

[8] P.E. Bjørstad and O.B. Widlund, Iterative methods for the solution of elliptic problems on regions partitioned into substructures. SIAM J. Numer. Anal. 23 (1986) 1093-1120.

[9] D. Brands, A. Klawonn, O. Rheinbach and J. Schröder, Modelling and convergence in arterial wall simulations using a parallel FETI solution strategy. Comput. Methods Biomech. Biomed. Eng. 11 (2008) 569-583.

[10] M. Dryja, A method of domain decomposition for three-dimensional finite element elliptic problem, in First International Symposium on Domain Decomposition Methods for Partial Differential Equations (Paris, 1987), SIAM, Philadelphia (1988) 43-61.

[11] M. Dryja, B.F. Smith and O.B. Widlund, Schwarz analysis of iterative substructuring algorithms for elliptic problems in three dimensions. SIAM J. Numer. Anal. 31 (1994) 1662-1694.

[12] C. Farhat and J. Mandel, The two-level FETI method for static and dynamic plate problems - part I: An optimal iterative solver for biharmonic systems. Comput. Methods Appl. Mech. Eng. 155 (1998) 129-152. 
[13] C. Farhat and F.-X. Roux, A method of Finite Element Tearing and Interconnecting and its parallel solution algorithm. Int. J. Numer. Meth. Eng. 32 (1991) 1205-1227.

[14] C. Farhat and F.-X. Roux, Implicit parallel processing in structural mechanics, in Computational Mechanics Advances 2, J. Tinsley Oden Ed., North-Holland (1994) 1-124.

[15] C. Farhat, J. Mandel and F.X. Roux, Optimal convergence properties of the FETI domain decomposition method. Comput. Methods Appl. Mech. Eng. 115 (1994) 367-388.

[16] C. Farhat, M. Lesoinne and K. Pierson, A scalable dual-primal domain decomposition method. Numer. Lin. Alg. Appl. 7 (2000) 687-714.

[17] C. Farhat, K.H. Pierson and M. Lesoinne, The second generation of FETI methods and their application to the parallel solution of large-scale linear and geometrically nonlinear structural analysis problems. Comput. Meth. Appl. Mech. Eng. 184 (2000) 333-374.

[18] C. Farhat, M. Lesoinne, P. Le Tallec, K. Pierson and D. Rixen, FETI-DP: A dual-primal unified FETI method - part I: A faster alternative to the two-level FETI method. Int. J. Numer. Meth. Eng. 50 (2001) 1523-1544.

[19] P. Gosselet and C. Rey, Non-overlapping domain decomposition methods in structural mechanics. Arch. Comput. Methods Eng. 13 (2006) 515-572.

[20] G.A. Holzapfel, Nonlinear Solid Mechanics. A continuum approach for engineering. Wiley (2000).

[21] A. Klawonn and O. Rheinbach, A parallel implementation of Dual-Primal FETI methods for three dimensional linear elasticity using a transformation of basis. SIAM J. Sci. Comput. 28 (2006) 1886-1906.

[22] A. Klawonn and O. Rheinbach, Inexact FETI-DP methods. Int. J. Numer. Methods Eng. 69 (2007) 284-307.

[23] A. Klawonn and O. Rheinbach, Robust FETI-DP methods for heterogeneous three dimensional elasticity problems. Comput. Methods Appl. Mech. Eng. 196 (2007) 1400-1414.

[24] A. Klawonn and O. Rheinbach, Highly scalable parallel domain decomposition methods with an application to biomechanics. Z. Angew. Math. Mech. (ZAMM) 90 (2010) 5-32.

[25] A. Klawonn and O.B. Widlund, FETI and Neumann-Neumann iterative substructuring methods: connections and new results. Commun. Pure Appl. Math. 54 (2001) 57-90.

[26] A. Klawonn and O.B. Widlund, Dual-Primal FETI Methods for Linear Elasticity. Commun. Pure Appl. Math. LIX (2006) $1523-1572$.

[27] A. Klawonn, O.B. Widlund and M. Dryja, Dual-Primal FETI methods for three-dimensional elliptic problems with heterogeneous coefficients. SIAM J. Numer. Anal. 40 (2002) 159-179.

[28] A. Klawonn, O. Rheinbach and O.B. Widlund, Some computational results for dual-primal FETI methods for elliptic problems in 3D, in Proceedings of the 15th international domain decomposition conference, R. Kornhuber, R.H.W. Hoppe, J. Périaux, O. Pironneau, O.B. Widlund and J. Xu Eds., Springer LNCSE, Lect. Notes Comput. Sci. Eng., Berlin (2005) 361-368.

[29] A. Klawonn, L.F. Pavarino and O. Rheinbach, Spectral element FETI-DP and BDDC preconditioners with multi-element subdomains. Comput. Meth. Appl. Mech. Eng. 198 (2008) 511-523.

[30] A. Klawonn, O. Rheinbach and O.B. Widlund, An analysis of a FETI-DP algorithm on irregular subdomains in the plane. SIAM J. Numer. Anal. 46 (2008) 2484-2504.

[31] A. Klawonn, P. Neff, O. Rheinbach and S. Vanis, Notes on FETI-DP domain decomposition methods for P-elasticity. Technical report, Universität Duisburg-Essen, Fakultät für Mathematik, http://www.numerik.uni-due.de/publications.shtml (2010).

[32] A. Klawonn, P. Neff, O. Rheinbach and S. Vanis, Solving geometrically exact micromorphic elasticity with a staggered algorithm. GAMM Mitteilungen 33 (2010) 57-72.

[33] U. Langer, G. Of, O. Steinbach and W. Zulehner, Inexact data-sparse boundary element tearing and interconnecting methods. SIAM J. Sci. Comput. 29 (2007) 290-314.

[34] P. Le Tallec, Numerical methods for non-linear three-dimensional elasticity, in Handbook of numerical analysis 3, J.L. Lions and P. Ciarlet Eds., Elsevier (1994) 465-622.

[35] J. Li and O.B. Widlund, FETI-DP, BDDC and Block Cholesky Methods. Int. J. Numer. Methods Eng. 66 (2006) $250-271$.

[36] J. Mandel and R. Tezaur, Convergence of a Substructuring Method with Lagrange Multipliers. Numer. Math. 73 (1996) $473-487$.

[37] J. Mandel and R. Tezaur, On the convergence of a dual-primal substructuring method. Numer. Math. 88 (2001) 543-558.

[38] P. Neff, On Korn's first inequality with nonconstant coefficients. Proc. Roy. Soc. Edinb. A 132 (2002) 221-243.

[39] P. Neff, Finite multiplicative plasticity for small elastic strains with linear balance equations and grain boundary relaxation. Contin. Mech. Thermodyn. 15 (2003) 161-195.

[40] P. Neff, A geometrically exact viscoplastic membrane-shell with viscoelastic transverse shear resistance avoiding degeneracy in the thin-shell limit. Part I: The viscoelastic membrane-plate. Z. Angew. Math. Phys. (ZAMP) 56 (2005) 148-182.

[41] P. Neff, Local existence and uniqueness for a geometrically exact membrane-plate with viscoelastic transverse shear resistance. Math. Meth. Appl. Sci. (MMAS) 28 (2005) 1031-1060.

[42] P. Neff, Local existence and uniqueness for quasistatic finite plasticity with grain boundary relaxation. Quart. Appl. Math. 63 (2005) 88-116.

[43] P. Neff, Existence of minimizers for a finite-strain micromorphic elastic solid. Proc. Roy. Soc. Edinb. A 136 (2006) $997-1012$.

[44] P. Neff, A finite-strain elastic-plastic Cosserat theory for polycrystals with grain rotations. Int. J. Eng. Sci. 44 (2006) $574-594$. 
[45] P. Neff and S. Forest, A geometrically exact micromorphic model for elastic metallic foams accounting for affine microstructure. Modelling, existence of minimizers, identification of moduli and computational results. J. Elasticity 87 (2007) $239-276$.

[46] P. Neff and I. Münch, Simple shear in nonlinear Cosserat elasticity: bifurcation and induced microstructure. Contin. Mech. Thermodyn. 21 (2009) 195-221.

[47] W. Pompe, Korn's first inequality with variable coefficients and its generalizations. Comment. Math. Univ. Carolinae 44 (2003) 57-70.

[48] A. Quarteroni and A. Valli, Numerical Approxiamtion of Partial Differential Equations, in Computational Mathematics 23, Springer Series, Springer, Berlin (1991).

[49] A. Quarteroni and A. Valli, Domain Decomposition Methods for Partial Differential Equations. Oxford Science Publications, Oxford (1999).

[50] J. Schröder and P. Neff, Invariant formulation of hyperelastic transverse isotropy based on polyconvex free energy functions. Int. J. Solids Struct. 40 (2003) 401-445.

[51] J. Schröder, P. Neff and D. Balzani, A variational approach for materially stable anisotropic hyperelasticity. Int. J. Solids Struct. 42 (2005) 4352-4371.

[52] J. Schröder, P. Neff and V. Ebbing, Anisotropic polyconvex energies on the basis of crystallographic motivated structural tensors. J. Mech. Phys. Solids 56 (2008) 3486-3506.

[53] B.F. Smith, P.E. Bjørstad and W. Gropp, Domain Decomposition: Parallel Multilevel Methods for Elliptic Partial Differential Equations. Cambridge University Press, Cambridge (1996).

[54] E.N. Spadaro, Non-uniqueness of minimizers for strictly polyconvex functionals. Arch. Rat. Mech. Anal. 193 (2009) 659-678.

[55] A. Toselli and O. Widlund, Domain Decomposition Methods - Algorithms and Theory, Springer Series in Computational Mathematics 34. Springer (2004).

[56] T. Valent, Boundary Value Problems of Finite Elasticity. Springer, Berlin (1988).

[57] K. Weinberg and P. Neff, A geometrically exact thin membrane model-investigation of large deformations and wrinkling. Int. J. Num. Meth. Eng. 74 (2007) 871-893.

[58] O.B. Widlund, An extension theorem for finite element spaces with three applications, in Proceedings of the Second GAMMSeminar, Kiel January 1986, Notes on Numerical Fluid Mechanics 16, Friedr. Vieweg und Sohn, Braunschweig/Wiesbaden (1987) 110-122. 